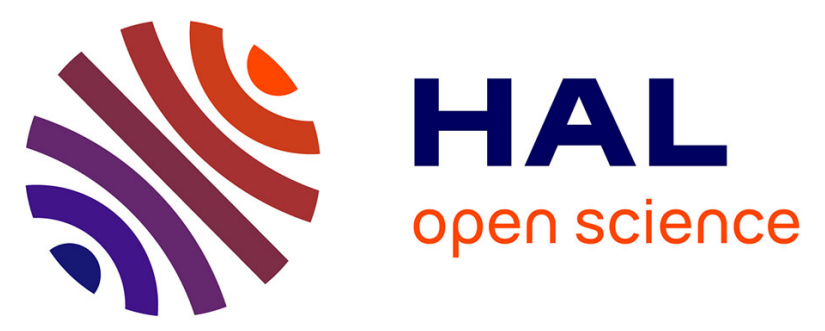

\title{
miR-4510 blocks hepatocellular carcinoma development through RAF1 targeting and RAS/RAF/MEK/ERK signalling inactivation
}

Amani Ghousein, Nicola Mosca, Flora Cartier, Justine Charpentier, Jean-William Dupuy, Anne-Aurélie Raymond, Paulette Bioulac-Sage, Christophe F Grosset

\section{To cite this version:}

Amani Ghousein, Nicola Mosca, Flora Cartier, Justine Charpentier, Jean-William Dupuy, et al.. miR-4510 blocks hepatocellular carcinoma development through RAF1 targeting and RAS/RAF/MEK/ERK signalling inactivation. Liver International, 2019, 40 (1), pp.240-251. 10.1111/liv.14276 . inserm-02437915

\section{HAL Id: inserm-02437915 https://www.hal.inserm.fr/inserm-02437915}

Submitted on 14 Jan 2020

HAL is a multi-disciplinary open access archive for the deposit and dissemination of scientific research documents, whether they are published or not. The documents may come from teaching and research institutions in France or abroad, or from public or private research centers.
L'archive ouverte pluridisciplinaire HAL, est destinée au dépôt et à la diffusion de documents scientifiques de niveau recherche, publiés ou non, émanant des établissements d'enseignement et de recherche français ou étrangers, des laboratoires publics ou privés. 


\section{Liver INTERNATIONM}

miR-4510 Blocks Hepatocellular Carcinoma Development Through RAF1 Targeting and RAS/RAF/MEK/ERK Signaling Inactivation

\begin{tabular}{|c|c|}
\hline Journal: & Liver International \\
\hline Manuscript ID & LIVint-19-00426.R1 \\
\hline Wiley - Manuscript type: & Original Articles \\
\hline $\begin{array}{r}\text { Date Submitted by the } \\
\text { Author: }\end{array}$ & 20-Sep-2019 \\
\hline Complete List of Authors: & $\begin{array}{l}\text { Ghousein, Amani; INSERM U1035, Biothérapies des Maladies } \\
\text { Génétiques, Inflamamtoires et Cancer } \\
\text { Mosca, Nicola; INSERM U1035, Biothérapies des Maladies Génétiques, } \\
\text { Inflamamtoires et Cancer } \\
\text { Cartier, Flora; INSERM U1035, Biothérapies des Maladies Génétiques, } \\
\text { Inflamamtoires et Cancer } \\
\text { Charpentier, Justine; INSERM U1035, Biothérapies des Maladies } \\
\text { Génétiques, Inflamamtoires et Cancer } \\
\text { Dupuy, Jean-William; Centre de Génomique Fonctionnelle de Bordeaux, } \\
\text { Plateforme Proteome } \\
\text { Raymond, anne-aurélie; Universite de Bordeaux, Inserm U1053 } \\
\text { Bioulac-Sage, Paulette; university Bordeaux, Inserm U1053 } \\
\text { Grosset, Christophe; Universite de Bordeaux, Inserm, BMGIC, U1035 }\end{array}$ \\
\hline Keywords: & hepatocellular carcinoma, proteomic, RAF1, ERK Signaling, miR-4510 \\
\hline
\end{tabular}

\section{SCHOLARONE Manuscripts}


1

2

3

4

5

6

7 'Univ. Bordeaux, Inserm, BMGIC, U1035, 33076 Bordeaux, France.

8 2Univ. Bordeaux, Centre de Génomique Fonctionnelle de Bordeaux, Plateforme Proteome, Bordeaux, 9 France.

10 UUniv. Bordeaux, Inserm, BARITON, U1053, 33076 Bordeaux, France.

11 ㅇRCID ID: http://orcid.org/0000-0003-2697-9352

12 5ORCID ID: https://orcid.org/0000-0002-0479-6291

13

14 Contact information : Christophe F. Grosset, Université de Bordeaux, INSERM, BMGIC, U1035, 146, Rue

15 Leo Saignat, 33076 Bordeaux Cedex, France; E-mail: christophe.grosset@inserm.fr; Tel.: +33 557574 630;

16 Fax: +33 557571374 .

17

18 Electronic word count: 4998 words

19

20 Total number of Figures and Tables: 5

21

\section{Abbreviations}

23 CAM, chorioallantoic membrane; CCNB1, Cyclin B1; CDK, cyclin-dependent kinase; CTRL, small RNA 24 control; ERK, extracellular signal-regulated kinase; FOS, c-Fos proto-oncogene; GPC3, glypican-3; HCC, 
1 hepatocellular carcinoma; miR-4510, mature microRNA-4510; miRNA, microRNA; mRNA, messenger RNA;

2 NTL, nontumoral liver; RAF1, RAF proto-oncogene serine/threonine-protein kinase; SMARC, SWI/SNF-

3 related matrix-associated actin-dependent regulator of chromatin; UTR, untranslated region.

4

5 Conflict of interest: The authors declare that they have no conflict of interest.

6

$7 \quad$ Statement of financial support

8 This study was supported by grants from L'Association pour le Développement du Liban to AG, La Ligue

9 Régionale Contre le Cancer (Comités Dordogne; Comité Gironde) to AG and CFG, the French State in the

10 framework of the "Investments for the future" Programme IdEx Bordeaux (grant ANR-10-IDEX-03-02) to

11 NM and La region Nouvelle-Aquitaine to NM.

13 Acknowledgements

14 We thank Véronique Guyonnet-Duperrat from the Vectorologie Platform of Bordeaux University and

15 Francis Sagliocco for his helpful technical assistance. We also thank Jean-Max Pasquet for providing

16 materials and Martin Hagedorn for critical comments on the manuscript. The flow cytometry and histology

17 were done in the Cytometry and Histology platforms, respectively, two service units of the TBM Core and

18 Bordeaux University.

20 Abstract

21 Background: Therapeutic outcomes using the multikinase inhibitors sorafenib or regorafenib remain 22 unsatisfactory for patients with advanced hepatocellular carcinoma (HCC). Thus, new drug modalities are 23 needed. We recently reported the remarkable capacity of miR-4510 to impede the growth of HCC and 24 hepatoblastoma through Glypican-3 (GPC3) targeting and Wnt pathway inactivation. Methods: To identify 
1 new targets of miR-4510, we used a label-free proteomic approach and reported down-regulation of RAF

2 proto-oncogene serine/threonine-protein kinase (RAF1) by miR-4510. Because the tumorigenic role of

3 RAF1 in HCC is controversial, we further studied RAF1:miR-4510 interactions using cellular, molecular as

4 well as functional approaches and a chicken chorioallantoic membrane (CAM) xenograft model. Results:

5 We found an increase in RAF1 protein in $59.3 \%$ of HCC patients and a specific up-regulation of its transcript

6 in proliferative tumors. We showed that miR-4510 inactivates the RAS/RAF/MEK/ERK pathway and

7 reduces the expression of downstream targets (i.e., c-Fos proto-oncogene [FOS]) through RAF1 direct

8 targeting. At a cellular level, miR-4510 inhibited HCC cell proliferation and migration and induced

9 senescence in part by lowering RAF1 messenger RNA (mRNA) and protein expression. Finally, we

10 confirmed the pro-tumoral function of RAF1 protein in HCC cells and its ability to sustain HCC tumor

11 progression in vitro and in vivo. Conclusions: In this work we confirm that RAF1 acts as an oncogene in

$12 \mathrm{HCC}$ and further demonstrate that miR-4510 acts as a strong tumor suppressor in the liver by targeting

13 many proto-oncogenes, including GPC3 and RAF1, and subsequently controlling key biological and

14 signaling pathways among which Wnt and RAS/RAF/MEK/ERK signals.

16 Keywords: liver; hepatocellular carcinoma; miR-4510; RAF1; RAS/RAF/MEK/ERK signaling.

18 Lay summary:

19 We previously reported that miR-4510 acts as a powerful antitumor molecule. In this new work, we

20 aimed to better understand how miR-4510 molecularly acts to inhibit liver tumor development. We found 21 that miR-4510 limits the oncogenic effect of RAF1 protein by lowering its expression and its functional 22 impact in liver cancer. 


\section{INTRODUCTION}

2 With approximately 782,000 new cases diagnosed each year in the world, hepatocellular carcinoma

3 ( $\mathrm{HCC}$ ) is the most frequent primary malignant liver tumor and is characterized by a very poor prognosis (5-

4 year survival $<15 \%$ ), dismal outcomes, and a steadily increasing incidence [1]. The main risk factors for HCC

5 are chronic liver diseases due to hepatotropic viruses (hepatitis B and C), alcohol consumption, and

6 metabolic syndromes [1]. Only one-third of patients with stage 0-A tumor can benefit from middle-term

7 curative regimens, including surgical resection, local ablation, and liver transplantation [1, 2]. For more

8 advanced diseases, the only approved drugs are sorafenib and regorafenib, two multikinase inhibitors that

9 increase patients' overall survival by approximately 3 months [3, 4]. Reasons for a failure of targeted

10 chemotherapies in advanced HCC are various and partially known. Some might include the lack of clear

11 driver-mutation identification in the treated population, complex molecular crosstalk between pro-

12 oncogenic pathways, high intratumor heterogeneity, drug resistance, and underlying hepatic dysfunctions

$13[1,2]$. Fortunately, many new drugs comprising immune checkpoint inhibitors, virus-based systems,

14 inhibitors of Hepatocyte growth factor receptor (MET) and mammalian target of rapamycin (mTOR), or

15 anti-angiogenic agents are currently under clinical evaluation for HCC $[3,4]$. Future advances in HCC

16 treatment likely reside in the combined use of multitarget drugs or therapeutic approaches (transarterial

17 chemoembolization plus chemotherapy) $[3,4]$ and in the development of new drug delivery vehicles (e.g., 18 extracellular vesicles and nanoparticles) or smart pro-drugs presenting both high antitumor property and

19 weak toxicity for the normal tissue compared to the uncoupled drugs $[5,6]$.

20 MicroRNAs (miRNAs) are short noncoding RNAs whose function is to post-transcriptionally regulate

21 gene expression. miRNA-mediated regulation occurs through sequence-specific binding to the coding or 22 untranslated regions (UTRs) of target messenger RNA (mRNA) [7, 8]. Thus, the miRNA-mRNA duplex 23 formation triggers translational repression and/or deadenylation-dependent mRNA decay and ultimately 24 reduces protein output. Approximately 2,500 miRNAs control thousands of genes involved in normal and 
1 pathological biological processes, including development, differentiation, proliferation, migration, 2 inflammation, and tumorigenesis. Compelling evidences have implied miRNAs as true drivers of liver 3 tumorigenesis in HCC [7] and hepatoblastoma, a primary liver tumor in early childhood [9]. miRNAs finely

4 tune many cellular processes that are fundamental for normal hepatocytes or hepatoma cells [7, 10].

5 Although the effect mediated by one miRNA on any particular target is modest, the simultaneous 6 regulation of a broad array of transcripts can lead to profound phenotypical changes and gene 7 reprograming $[11,12]$. Due to their functional redundancy and multitargeting ability, adequately 8 formulated miRNAs might offer new therapeutic perspectives for patients presenting with advanced HCC $9 \quad[1,5,11-13]$.

Recently, we reported for the first time the tumor-suppressive role of mature microRNA 4510 (miR-

11 4510) in both HCC and hepatoblastoma [10]. Our data showed that miR-4510 is decreased in hepatic 12 tumors, blocks the growth and proliferation of cancerous hepatic cells in vitro and in vivo, induces 13 apoptosis, and inhibits Wnt signaling and the expression of downstream targets, including $B M P 4, D K K 1$, 14 LEF1, and TCF7L1. The blocking of endogenous miR-4510 with a specific antimiR also increased HCC cell 15 growth [10]. Part of the phenotypical changes mediated by miR-4510 in HCC cells was dependent on 16 Glypican-3 (GPC3) expression, the only validated miR-4510 target reported so far [10]. To gain further 17 insights into the tumor-suppressive role of miR-4510 in the liver, we sought to find new targets of this 18 miRNA using a label-free proteomic approach. After validating the decreased expression of several 19 oncogenes by miR-4510, we focused our interest on RAF proto-oncogene serine/threonine-protein kinase 20 (RAF1, also known as C-RAF), an intermediate member of the mitogen-activated protein 21 kinase/extracellular signal-regulated kinase (MAPK/ERK) signaling pathway (also known as 22 RAS/RAF/MEK/ERK pathway) with controversial oncogenic function in HCC [14-18]. We report the direct 23 regulation of RAF1 by miR-4510 in HCC cells and further confirm the oncogenic property of this 24 serine/threonine kinase in the pathogenesis of HCC. 


\section{METHODS}

\section{Patient samples}

3 Tissues were snap frozen and stored at $-80^{\circ} \mathrm{C}$. Samples were obtained with written informed

4 consent, and the study protocol was approved by the ethics committee of Bordeaux Liver Bank (project 5 \#BB-0033-00036). Liver samples were clinically, histologically, and genetically characterized (Supporting

6 Table S1). Liver tissues were immediately frozen in liquid nitrogen and stored at $-80^{\circ} \mathrm{C}$ until used for 7 molecular studies.

\section{Cell culture, lentiviral transduction, and transfection by small RNAs}

10 Human HCC-derived cell lines Huh7 and Hep3B were cultured in Dulbecco's modified Eagle's 11 medium (DMEM) (4.5 g/L; Invitrogen, Carlsbad, CA, USA), supplemented with $10 \%$ fetal bovine serum, $12100 \mathrm{U} / \mathrm{mL}$ penicillin, and $100 \mu \mathrm{g} / \mathrm{mL}$ streptomycin, maintained at $37^{\circ} \mathrm{C}$ in a humidified atmosphere of $5 \%$

$13 \mathrm{CO}$, and harvested with $0.05 \%$ trypsin-0.03\% ethylene diamine tetra-acetic acid (EDTA) [19]. Cell line

14 authentication was performed every year using short tandem repeat analysis, and absence of mycoplasma

15 contamination in cell culture media was tested every month. Stable Huh7 cells coexpressing tomato

16 transgene and green fluorescent protein (GFP) transgene harboring the wild-type (WT) or mutated (MUT)

17 RAF-1 3'-UTR were generated by lentiviral transduction using a multiplicity of infection (MOI) of 1 . Stable

18 Huh7 and Hep3B cells expressing human RAF1 were generated by lentiviral transduction using an MOI of

19 5. Production and titration of infectious lentiviral particles, cell transduction, as well as biosafety

20 considerations and procedures have been described [19]. Cell transfections were done as described [20]

21 using the following small RNAs: miR-4510 mimic as well as small interfering RNA against RAF1 (siRAF1)

22 (Sigma-Aldrich, St Louis, MO, USA) and AllStars Negative Control small interfering RNA (siRNA) (Qiagen, 23 Hilden, Germany). 


\section{Sample preparation for proteomic analysis}

2 This analysis was performed by the proteomics core facility at University of Bordeaux. The steps of

3 sample preparation and protein digestion were performed as described [21].

\section{$5 \quad$ Nano liquid chromatography-tandem mass spectrometry analysis}

6 Online nano liquid chromatography-tandem mass spectrometry (LC-MS/MS) analyses were

7 performed using an Ultimate 3000 RSLC Nano-UPHLC system (Thermo Scientific, USA) coupled to a

8 nanospray Q Exactive hybrid quadrupole-Orbitrap mass spectrometer (Thermo Scientific, USA). Then, 500

9 ng of each peptide extract was loaded on a 300- $\mu \mathrm{m}$ ID $\times 5$ mm PepMap C18 precolumn (Thermo Scientific,

$10 \mathrm{USA}$ ) at a flow rate of $10 \mu \mathrm{L} /$ minute. After a 3-minute desalting step, peptides were separated on a $75-\mu \mathrm{m}$

11 ID $\times 25 \mathrm{~cm}$ C18 Acclaim PepMap RSLC column (Thermo Scientific, USA) with a $4 \%$ to $40 \%$ linear gradient of 12 solvent B ( $0.1 \%$ formic acid in $80 \%$ acetonitrile) for 108 minutes. The separation flow rate was set to 300

$13 \mathrm{~nL} /$ minute. The mass spectrometer operated in positive ion mode at a 1.8-kV needle voltage. Data were

14 acquired using Xcalibur 3.1 software in a data-dependent mode. MS scans (m/z 300-1600) were recorded

15 at a resolution of $R=70000$ (at $\mathrm{m} / \mathrm{z} 200$ ) and an Automatic gain control (AGC) target of $3 \times 106$ ions

16 collected within 100 ms. Dynamic exclusion was set to 30 seconds, and top 12 ions were selected from

17 fragmentation in higher-energy C-trap dissociation (HCD) mode. MS/MS scans with a target value of $1 \times$ 18105 ions were collected with a maximum fill time of $100 \mathrm{~ms}$ and a resolution of $\mathrm{R}=17500$. Additionally,

19 only +2 and +3 charged ions were selected for fragmentation. Other settings included no sheath and no 20 auxiliary gas flow; heated capillary temperature, $200^{\circ} \mathrm{C}$; normalized $\mathrm{HCD}$ collision energy of $27 \mathrm{eV}$; and an 21 isolation width of $2 \mathrm{~m} / \mathrm{z}$.

22

\section{Database search and processing}


$1 \quad$ For protein identification, Sequest HT and Mascot 2.4 algorithms through Proteome Discoverer 1.4

2 Software (Thermo Fisher Scientific, Inc.) were used for protein identification in batch mode by searching

3 against a Homo sapiens protein database (68,421 entries, Reference Proteome Set, release 2015_04;

4 http://www.uniprot.org/website). Two missed enzyme cleavages were allowed. Mass tolerances in MS

5 and MS/MS were set to $10 \mathrm{ppm}$ and $0.02 \mathrm{Da}$. Oxidation of methionine, acetylation of lysine, and 6 deamidation of asparagine and glutamine were searched as dynamic modifications.

7 Carbamidomethylation on cysteine was searched as static modification. Peptide validation was performed

8 using Percolator algorithm, and only "high confidence" peptides were retained corresponding to a $1 \%$ false

9 discovery rate (FDR) at peptide level [22].

\section{Label-Free Quantitative Data Analysis}

12 Raw LC-MS/MS data were imported in Progenesis QI (version 2.0; Nonlinear Dynamics, a Waters

13 Company) for feature detection, alignment, and quantification. All sample features were aligned according

14 to retention times by manually inserting up to 50 landmarks followed by automatic alignment to maximally

15 overlay all the 2-dimensional ( $\mathrm{m} / \mathrm{z}$ and retention time) feature maps. Singly charged ions and ions with

16 higher charge states than 6 were excluded from analysis. All remaining features were used to calculate a

17 normalization factor for each sample that corrects for experimental variation. Peptide identifications (with

18 FDR <1\%) were imported into Progenesis. Only nonconflicting features and unique peptides were

19 considered for calculation of quantification at protein level. A minimum of two peptides matched to a 20 protein was used as the criterion for identification as a differentially expressed protein. We applied a $t$ test

21 (Welch's $t$ test that does not assume equal population variance) to miR-4510 versus control. We set the 22 threshold for the significance of the adjusted $P$ value at 0.05 . The MS proteomics data have been deposited 23 to the ProteomeXchange Consortium through the PRIDE [23] partner repository with the data set identifier $24 \quad$ PXD010085. 


\section{Antibodies, immunoblot analysis}

3 Cells were lysed in RIPA lysis buffer (Sigma, St Louis, MO, USA) supplemented with protease inhibitor

4 cocktail (Roche Diagnostics, Mannheim, Germany), and protein concentration was determined using the 5 Pierce BCA Protein Assay Kit (Thermo Scientific, Rockford, USA). Approximately $40 \mu \mathrm{g}$ of proteins from 6 each sample were separated on $4 \%$ to $15 \%$ precast polyacrylamide gel (Bio-Rad, Hercules, CA, USA) and 7 blotted onto nitrocellulose membranes $(0.2 \mu \mathrm{m}$ nitrocellulose, Bio-Rad) using Trans-Blot Turbo Transfer 8 System (Bio-Rad). The membranes were blocked in 5\% skim milk and then incubated with each of the 9 following specific primary antibodies: rabbit anti-cyclin-dependent kinase 6 (CDK6) (1:1000, SC-177, Santa 10 Cruz Biotechnology); rabbit anti- S-phase kinase-associated protein 2 (SKP2) (1:1000, \#4358, Cell Signaling 11 Technology); rabbit anti-cyclin B1 (CCNB1) (1:1000, GTX100911, Gene Tex); mouse anti-RAF1 (1:1000, SC12 7267, Santa Cruz Biotechnology); mouse anti-ERK (1:2000, \#4696, Cell Signaling Technology); rabbit anti13 phospho-ERK (p-ERK) (1:1000, \#4377, Cell Signaling Technology); rabbit anti-CFOS (1:1000, SC-52, Santa 14 Cruz Biotechnology); mouse anti-Myc proto-oncogene protein (MYC) (1:1000, SC-40, Santa Cruz 15 Biotechnology); mouse anti-GPC3 (1:1000, B0025R, Biomosaics); and rabbit anti-glyceraldehyde 316 phosphate dehydrogenase [GAPDH] (1:2000, SC-25778, Santa Cruz Biotechnology) at $4^{\circ} \mathrm{C}$ overnight. All 17 blots were analyzed with the Odyssey system (Li-Cor Biosciences).

19 Chick chorioallantoic membrane assay

20 Fertilized embryos were received at the stage of segmentation and then incubated at $37.4^{\circ} \mathrm{C}$ and $70 \%$ 21 humidity. At day 3 of development, the eggshell was opened on the top and the opening sealed with 22 medical-grade Durapore tape. At day 10 of embryonic development, 1 million Huh7 cells were embedded 23 in Matrigel (growth-factor reduced, Corning, Corning, NY, USA) droplets (40 $\mu \mathrm{L}$ ) and deposited on the 24 chorioallantoic membrane (CAM). Pictures of experimental tumors were taken every day (until day 16 of 
1 embryonic development) using a stereomicroscope (SMZ745T) and camera (DS-Fi2) from Nikon and then 2 analyzed with the NSI Element D software (Nikon, Tokyo, Japan). At day 16 of embryonic development, all

3 tumors were formalin-fixed and weighed using a precision balance after they were further processed for 4 histology.

5

6 Bioinformatic and statistical analyses

7 miR-4510 target selection was done using a score $>-0.3$ for TargetScan $7.2[24]$ and a seed match $=1$ and

8 an energy <-22.0 for miRWalk 3.0 (http://mirwalk.umm.uni-heidelberg.de) [25]. All data are displayed as

9 a mean of three or more independent experiments, and error bars indicate standard deviation of the

10 mean. Statistical analyses were performed using GraphPad Prism 5.0 or 6.0 software as described [10].

11 When an experiment contained two unmatched groups of values, the nonparametric Mann-Whitney test

12 was used for the comparison of means. When an experiment contained two paired groups, the 2-tailed

13 Wilcoxon matched-pairs signed ranked test was used. When an experiment contained three groups of

14 values or more, regular one-way or two-way analysis of variance (ANOVA) was used for the comparison

15 of multiple means. The one-way ANOVA test was followed by the Dunnett's multiple-comparison post-

16 test when all data were compared to control or by the Bonferroni's multiple-comparison post-test when

17 all data were compared. The two-way ANOVA test was followed by the Tuckey's multiple-comparison post-

18 test when all data were compared to control. The $P$ value is indicated at the bottom of each figure legend.

19 In each figure, the number of independent experiments ( $n$ ) and the ANOVA $P$ value is indicated in brackets.

20 Results were considered significant when $P<0.05$. For all data in figures: $* P<0.05 ; * * P<0.01 ; * * * P<$

210.001.

22 See supporting Materials and Methods for additional details. 


\section{RESULTS}

\section{Identification of new targets of miR-4510 in HCC cells}

3 To find new targets of miR-4510, we transfected HCC-derived Huh7 cells with a synthetic miR-4510

4 mimic or a small RNA control (CTRL), extracted total proteins 24 hours later, and comparatively analyzed

5 cellular proteomes using a label-free approach. LC-MS/MS spectra analyses detected 3,300 proteins and,

6 according to a reproducible up- or down-regulation of $25 \%$ or more (with ratio $\geq 2$ or $\leq 0.5$ ), revealed 611

7 significantly deregulated proteins (322 down and 289 up) in miR-4510-transfected cells compared to

8 control cells (Fig. 1a; Supporting Tables S2 and S3). Among the 322 down-regulated proteins and possible

9 novel miR-4510 targets, 54 (TargetScan 7.2 [24]) and 23 (miRWalk 3.0 [25]) genes encoding for these

10 proteins were highly predicted to interact with miR-4510, and 9 genes (DRG2, GPI, LASP1, LIG3, RAB1B,

11 RAF1, RTN3, TPP1, UQCC1) were collectively found in proteomic data and mRNA:miRNA predictions (see

12 insert in Fig. 1a). Noticeably, many of the 322 decreased proteins are associated with cell cycle,

13 proliferation, survival, signaling, protein synthesis, metabolic pathways (lipids; amino and nucleic acids),

14 and genome regulation (Fig. 1b). The down-regulation of CDK6, S-phase kinase-associated protein 2

15 (SKP2), G2/mitotic-specific cyclin B1 (CCNB1), and RAF1 proteins in miR-4510-transfected cells was

16 confirmed by western blot (Fig. 1c), therefore validating our proteomic data (Fig. 1a). We previously

17 showed that miR-4510 inhibits cell proliferation by arresting HCC cells in GO and reducing their entry in S

18 phase [10]. Therefore, miR-4510 likely exerts its anti-proliferative function through the direct or indirect

19 down-regulation of CDK6, SKP2, and CCNB1. We also noticed that RAF1 was involved in many cellular and

20 molecular processes altered in miR-4510-transfected Huh7 cells (Fig. 1b; Supporting Table S4), suggesting

21 a central role of this serine/threonine-protein kinase in miR-4510 tumor-suppressive function. Altogether, 22 our results show that miR-4510 controls a large spectrum of genes in hepatic cells and decreases

23 expression of multiple well-known oncogenes, some comprising cell cycling effectors and cell signaling

24 mediators, among which RAF1 stands as a key effector. 


\section{Deregulation of RAF1 in HCC}

3 RAF1 encodes for the serine/threonine kinase RAF1 with intermediate regulatory functions in the

4 linear RAS/RAF/MEK/ERK pathway. Because RAF1 overexpression in HCC was still a matter of debate [14-

5 16], we measured its expression by western blot using total protein extracts from 27 patient samples

6 (Supporting Fig. S1) [10]. In our cohort, RAF1 protein was globally and significantly increased in HCC

7 compared to nontumoral liver (NTL) (Fig. 2a). Paired tumor/NTL ratios highlighted that RAF1 is

8 overexpressed in 16 samples (59.3\%), unchanged in 10 samples (37.0\%), and decreased in 1 sample (3.7\%)

9 (Fig. 2b). Disparity in RAF1 expression was also observed at a histological level using tissue sections from

10 a total of 9 patients, and cirrhotic tissues were sometimes stained with RAF1 protein (Fig. 2c; Supporting

11 Fig. S2), a result in agreement with a previous study [26]. Using publicly available transcriptomic data [27],

12 we also found a specific increase in RAF1 mRNA in proliferative HCC subgroups (G1, G3, and to a lesser

13 extend G2; 35.7\%) (Fig. 2d). Moreover, using the Human Protein Atlas databank

14 (www.proteinatlas.org/ENSG00000132155-RAF1), we found low expression of RAF1 protein in normal

15 liver tissue and a staining of hepatocyte nuclei. The transcriptomic analysis of a cohort of 365 cases

16 (www.proteinatlas.org/ENSG00000132155-RAF1/pathology/tissue/liver+cancer\#) with the best

17 separation (comprising the first quartile of tumors) showed a significant association $(P=0.00266)$ between

18 high expression level of RAF1 mRNA and poor survival of patients. Finally, correlation analysis of our cohort

19 of samples showed that RAF1 protein expression inversely correlates with miR-4510 (Fig. 2e). Altogether,

20 these data suggest that RAF1 expression is increased in a majority of HCC tumors, associated with poor

21 prognosis probably due to a decrease of miR-4510.

22

23 miR-4510 inhibits the RAS/RAF/MEK/ERK pathway by targeting RAF1 
1 To further study the impact of miR-4510 on RAF1 and RAS/RAF/MEK/ERK signaling, we measured 2 the expression of ERK, phospho-ERK, and the downstream targets FOS and MYC in Huh7 cells.

3 Complementary experiments were performed with cells transfected with an siRNA specifically silencing

$4 \quad$ RAF1. Results clearly showed that consecutively to a strong decrease of RAF1 mRNA (Fig. 3d) and protein 5 levels (Fig. 3a), miR-4510 triggered a dephosphorylation of ERK and a decline of FOS and MYC proto6 oncogene proteins (Fig. 3a-c). Similar results were observed with RAF1 siRNA (Fig. 3a-d). miR-4510 and 7 siRAF1 also dramatically decreased RAF1 expression in Hep3B, a second HCC cell line (Supporting Fig. S3).

8 To determine whether miR-4510 directly interacts with RAF1 3'-UTR, functional analyses were performed 9 using the fluorescent-reporter FunREG system [19, 20]. The predicted miR-4510 site located at position 10 219-225 in RAF1 3'-UTR was mutated by changing two cytidines in two guanines (r.221C>G and r.223C>G;

11 Fig. 3e). Compared to cells expressing eGFP-WT RAF1 3'-UTR transgene, these two base mutations totally 12 abolished the regulation of the fluorescent reporter gene by miR-4510 (Fig. 3e). Altogether, these data 13 demonstrated that miR-4510 inactivates RAS/RAF/MEK/ERK pathway in HCC cells by directly targeting 14 RAF1 3'-UTR at position 219-225.

RAF1 silencing phenocopies miR-4510-mediated antitumor effect in HCC cells

17 Because the oncogenic function of RAF1 in HCC was controversial [14-16], we investigated the 18 impact of RAF1 silencing in HCC cells using in vitro and in vivo approaches. Here, miR-4510 was used as 19 positive control for both cell growth inhibition and apoptosis induction [10]. RAF1 silencing decreased the 20 growing capacity of both Huh7 and Hep3B cells by slightly extending the G0/G1 phase and shortening the 21 S phase (Fig. 4a-b; Supporting Fig. S4). As expected, similar but stronger effects were observed with miR22 4510. Moreover, both RAF1 depletion and miR-4510 induced HCC cell senescence more efficiently than 23 the pro-senescent miRNA miR-624-5p [9], (Fig. 4c; Supporting Fig. S5) whereas only miR-4510 triggered 24 apoptosis in the two cell lines (Supporting Fig. S6). Interestingly, the combination of miR-4510 and 
1 sorafenib was more efficient to induce cell apoptosis than miR-4510 or sorafenib alone, and sorafenib

2 induced both miR-4510 increase and RAF1 protein reduction (Supporting Fig. S7). RAF1 silencing and miR-

34510 also significantly impeded the capacity of Huh7 cells to migrate (Fig. 4d; Supporting Fig. S8a-b). Next,

4 we investigated the impact of overexpressing a 3'-UTR-less RAF1 transgene in HCC cells (Supporting Figs.

5 S9 and S10) and found that RAF1 potentiates the growth of both Huh7 and Hep3B cells (Fig. 4e). In

6 addition, overexpression of RAF1 partly counteracted the anti-proliferation effect of miR-4510 in both cell

7 lines (Fig. 4e). Altogether, these data show that the tumor-suppressive role of miR-4510 is partly mediated

8 by RAF1 targeting and down-regulation.

9

10 RAF1 favors HCC tumor growth in vivo

11 Next, we investigated the impact of RAF1 depletion on HCC growth in vivo using chicken embryos

12 presented by the tumor CAM model (Fig. 5a) $[9,10,28]$. At a macroscopic level, either before or after

13 tumor extraction and formalin fixation, we noticed that engrafted Huh7 cells formed a tissue mass smaller

14 with siRAF1 compared to control in 6 days with no bleeding (Fig. 5b, lanes 1 and 2). This observation was

15 further confirmed by weighting resected tumors (Fig. 5c) and measuring tumor thickness using formalin-

16 fixed paraffin-embedded hematoxylin-eosin-saffron (HES)-stained tissue sections (Fig. 5b, middle lane; Fig.

17 5d; Supporting Fig. S11a-b). After 6 days of tumor development, the decrease of RAF1 staining in siRAF1

18 tumors compared to control was confirmed by histology (Fig. 5b, lane 4; Supporting Fig. S11c-d). Similar

19 growth inhibition and histological data were obtained in miR-4510 tumors compared to control [10], (Fig.

20 5e; Supporting Fig. S12a-d) confirming the RAF1 inhibitory ability of miR-4510 in this animal model.

21 Moreover, the concomitant decrease of Ki67 immunostaining in siRAF1 tumors compared to control (Fig.

$225 b$, bottom lane; Supporting Fig. S11e-f) showed that tumor cells were less abundant and less proliferative

23 following RAF1 silencing. Altogether, these data demonstrate that RAF1 acts as an oncogene in HCC and

24 supports tumoral hepatic cell growth in vivo. 


\section{DISCUSSION}

We recently reported the decrease of miR-4510 in pediatric and adult liver cancers and its antitumor

3 action through GPC3 regulation and Wnt pathway inactivation [10]. miR-4510 was also found to be down-

4 regulated in other malignancies, including bladder carcinoma [29], recurrent breast cancer [30], and

5 colorectal adenocarcinoma [31]. Therefore, the decrease of miR-4510 in a broad variety of cancers points

6 to its role as a general tumor suppressor. However, the only validated (oncogenic) target of miR-4510 is,

7 so far, GPC3 [10]. Here, we report that miR-4510 inhibits the RAS/RAF/MEK/ERK pathway in HCC cells by

8 directly targeting RAF1 through a site located in its 3'-UTR. Two additional miR-4510 sites were predicted

9 to be located in RAF1 5'-UTR (Supporting Fig. S13), explaining in part the very strong inhibitory effect of

10 miR-4510 on RAF1 mRNA and protein expression (Fig. 3a,d; Supporting Fig. S3). However, the biological

11 relevance of these sites was not tested in this work. Thus, these new data further confirm the antitumor

12 function of miR-4510 and its role as a negative regulator of cellular functions (e.g., proliferation, survival,

13 migration) through diminution of oncogenic signaling pathways among which Wnt/beta-catenin and

14 RAS/RAF/MEK/ERK pathways. In addition, the down-regulation of CDK6, SKP2,_and cyclin B1 proteins (and,

15 to a lesser extent, GAK, CDK2, and CDK1; Supporting Table S2) in miR-4510-transfected Huh7 cells explains

16 the strong and wide negative impact of miR-4510 in vitro and in vivo on both cell proliferation and cell

17 cycling (Fig. 4) [10]. However, none of these genes was highly predicted to directly interact with miR-4510

18 using TargetScan (data not shown). Additional oncogenes down-regulated by miR-4510 comprise SKP2, an

19 E3 ubiquitin ligase that participates in cell survival by lowering the susceptibility of tumor cells to MYC-

20 induced apoptosis [32], and SRPK1 (SRSF protein kinase 1), a splicing kinase involved in transcript

21 maturation, which participates in tumor angiogenesis, invasiveness, and progression [33]. Several 22 SWI/SNF-related, matrix-associated, actin-dependent regulator of chromatin (SMARC) proteins (e.g.,

23 SMARCC2 and SMARCD1 and, to a lesser extent, SMARCA4, SMARCC1, and SMARCE1; Supporting Table

24 S2) were also decreased in miR-4510-transfected Huh7 cells, suggesting a probable link between miR- 
14510, SWI/SNF complex, and chromatin remodeling. In the literature, SMARCD1 is documented as a

2 tumorigenic and metastatic protein in gastric cancer [34], and it prevents cellular senescence and lipid

3 accumulation in hepatocytes [35]. Intriguingly, numerous SMARC members are increased in

4 hepatoblastoma and more particularly in proliferative and Fanconi Anemia pathway-activated C2A tumors

5 [36]. Therefore, it is likely that the marked pro-senescent effect of miR-4510 observed in HCC cells (Fig. 4c)

6 is linked to its capacity to control SMARCC2, SMARCD1, and SWI/SNF complex activation.

7 The serine/threonine kinase RAF1 is an intermediate component of the RAS/RAF/MEK/ERK pathway

8 cascade (also known as MAPK/ERK pathway) and serves as a link between ligand-activated receptor

9 tyrosine kinases (e.g., epidermal growth factor receptor [EGFR], fibroblast growth factor receptor [FGFR],

10 platelet-derived growth factor receptor [PDGFR]) and nuclear gene reprogramming that modulates

11 hundreds of genes, including many protumoral transcriptional factors (e.g., MYC, FOS). Alterations of

12 RAS/RAF/MEK/ERK pathway, mostly due to activating mutations in RAS and BRAF genes, have been

13 documented in many solid tumors, including HCC, melanoma, and lung, colon, and prostate carcinomas as

14 well as hematological malignancies such as myeloid leukemia $[1,17,37]$. Because of its central position in

15 RAS/RAF/MEK/ERK pathway, RAF1 is tightly controlled in cells by both

16 phosphorylation/dephosphorylation mechanisms [17, 38] and miRNAs (e.g., miR-7-5p, miR-15a/b, miR-16,

17 and miR-195) [39, 40]. Thus, the regulation of RAF1 by miR-4510 has several cellular and

18 pathophysiological implications. First, RAF1 silencing by miR-4510 partly explains the remarkable

19 antitumoral effect of miR-4510 on hepatic cancer cells [10]. Second, because sorafenib- or regorafenib-

20 based therapies are not curative and only slightly increase patient life expectancy, patients with advanced

21 HCC who need alternative therapeutic solutions may benefit from a miR-4510-based therapy. In addition,

22 many mechanisms of resistance have been experienced in relapsed patients following treatment with

23 RAF1, MEK, or ERK inhibitors [38], emphasizing the need to combine multikinase inhibitors with other

24 categories of antitumor agents to block multiple oncogenic pathways at the same time. Finally, our in vitro 
1 data show that sorafenib provoked miR-4510 increase and RAF1 depletion. Moreover, the combination of 2 miR-4510 and sorafenib was more efficient to eliminate HCC cells compared to each molecule used alone

3 (Supporting Fig. S7). These data further confirm that sorafenib targets other kinases than RAF1 in cells and

4 support the idea that combining miR-4510 with sorafenib or regorafenib may resolve current limitations 5 due to chemoresistance and poor responsiveness of patients with advanced HCC [1].

6 Sorafenib is the standard of care for patients with advanced HCC, but unexpectedly, the dogma 7 about the oncogenic role of RAF1 in cancer was challenged by the study of Jeric and coworkers [14]. The 8 study showed that RAF1 is significantly down-regulated in HCC and negatively regulates liver 9 carcinogenesis and HCC cell proliferation [14]. Here, we provide evidence that RAF1 mRNA is specifically 10 increased in proliferative and poor-prognosis HCC displaying TP53 mutation, CDKN2A promoter 11 methylation, and chromosome instability [27] (Fig. 2d) In a cohort of non-selected 27 paired tumor/NTL 12 samples, we also found that RAF1 protein is increased in $59.3 \%$ of patients, unchanged in $37.0 \%$, and 13 decreased in 3.7\% (Fig. 2b). Data from the Human Protein Atlas databank further support our results and

14 report a weak expression of RAF1 protein in normal liver and a poor survival of HCC patients expressing 15 high level of RAF1. Finally, a study performed in Asian populations reported the up-regulation of RAF1 16 protein in $42.9 \%$ of liver cirrhosis and in $49.0 \%$ to $100 \%$ of $\operatorname{HCC}[26,41]$. Altogether, these data demonstrate 17 that (1) RAF1 expression is highly increased in HCC but variable from one patient to another, and (2) high 18 expression of RAF1 is associated with cell proliferation, chemoresistance, and tumor aggressiveness.

19 Therefore, any drug capable of blocking its expression and downstream biological pathways may be 20 beneficial to patients with advanced HCC, and miR-4510 stands as a significant candidate. The discrepancy 21 between our data and the work by Jeric and coworkers [14] remains unexplained at this stage and may be 22 due to the use of different cell types, animal models, and anti-RAF1 siRNAs.

23 In conclusion, our work confirms the oncogenic function of RAF1 in HCC and further highlights the 24 remarkable antitumor function of miR-4510 in HCC and its central position in the control of cell 
1 proliferation, survival, and mobility, making miR-4510 particularly relevant for miRNA-based therapies in 2 patients presenting advanced, unresectable, or drug-resistant liver cancer.

3

10

11

12

13

14

15

16

17

18 
1. Llovet, J.M., et al., Advances in targeted therapies for hepatocellular carcinoma in the genomic era. Nat Rev Clin Oncol, 2015.

2. Attwa, M.H. and S.A. El-Etreby, Guide for diagnosis and treatment of hepatocellular carcinoma. World J Hepatol, 2015. 7(12): p. 1632-51.

3. Sherman, M., Regorafenib for treatment of hepatocellular carcinoma. Hepatology, 2018. 67(3): p. 1162-1165.

4. Ding, X.X., et al., Precision medicine for hepatocellular carcinoma: driver mutations and targeted therapy. Oncotarget, 2017. 8(33): p. 55715-55730.

5. Varshosaz, J. and M. Farzan, Nanoparticles for targeted delivery of therapeutics and small interfering RNAs in hepatocellular carcinoma. World J Gastroenterol, 2015. 21(42): p. 12022-41.

6. Renoux, B., et al., Targeting the tumour microenvironment with an enzyme-responsive drug delivery system for the efficient therapy of breast and pancreatic cancers. Chem Sci, 2017. 8(5): p. 3427-3433.

7. Wang, X.W., N.H. Heegaard, and H. Orum, MicroRNAs in liver disease. Gastroenterology, 2012. 142(7): p. 1431-43.

8. Helwak, A., et al., Mapping the human miRNA interactome by CLASH reveals frequent noncanonical binding. Cell, 2013. 153(3): p. 654-65.

9. Indersie, E., et al., MicroRNA therapy inhibits hepatoblastoma growth in vivo by targeting beta-catenin and Wht signaling. Hepatol Commun, 2017. 1(2): p. 168-183.

10. Cartier, F., et al., New tumor suppressor microRNAs target glypican-3 in human liver cancer. Oncotarget, 2017. 8(25): p. 41211-41226.

11. Hayes, J., P.P. Peruzzi, and S. Lawler, MicroRNAs in cancer: biomarkers, functions and therapy. Trends Mol Med, 2014. 20(8): p. 460-9.

12. Li, Z. and T.M. Rana, Therapeutic targeting of microRNAs: current status and future challenges. Nat Rev Drug Discov, 2014. 13(8): p. 622-38.

13. Lam, J.K., et al., siRNA Versus miRNA as Therapeutics for Gene Silencing. Mol Ther Nucleic Acids, 2015. 4: p. e252.

14. Jeric, I., et al., A cell-autonomous tumour suppressor role of RAF1 in hepatocarcinogenesis. Nat Commun, 2016. 7: p. 13781.

15. Giambartolomei, S., et al., Sustained activation of the Raf/MEK/Erk pathway in response to EGF in stable cell lines expressing the Hepatitis C Virus (HCV) core protein. Oncogene, 2001. 20(20): p. 2606-10.

16. Wang, Z., et al., Grb2-associated binder-1 plays a central role in the hepatocyte growth factor enhancement of hepatoma growth inhibition by K vitamin analog compound 5. Hepatology, 2007. 46(6): p. 2003-13.

37

38

39

17. Yang, S. and G. Liu, Targeting the Ras/Raf/MEK/ERK pathway in hepatocellular carcinoma. Oncol Lett, 2017. 13(3): p. 1041-1047.

40

41

42

43

44

18. Schmitz, K.J., et al., Activation of the ERK and AKT signalling pathway predicts poor prognosis in hepatocellular carcinoma and ERK activation in cancer tissue is associated with hepatitis $C$ virus infection. J Hepatol, 2008. 48(1): p. 83-90.

19. Laloo, B., et al., Analysis of post-transcriptional regulations by a functional, integrated, and quantitative method. Mol Cell Proteomics, 2009. 8(8): p. 1777-88. 
1

11

12

13

14

15

16

17

18

19

20

21

22

23

24

25

26

27

28

29

30

31

32

33

34

35

36

37

38

39

40

41

42

43

44

45

20. Maurel, M., et al., A functional screening identifies five micrornas controlling glypican3: role of mir-1271 down-regulation in hepatocellular carcinoma. Hepatology, 2013. 57(1): p. 195-204.

21. Henriet, E., et al., Argininosuccinate synthase 1 (ASS1): A marker of unclassified hepatocellular adenoma and high bleeding risk. Hepatology, 2017. 66(6): p. 20162028.

22. Kall, L., et al., Semi-supervised learning for peptide identification from shotgun proteomics datasets. Nat Methods, 2007. 4(11): p. 923-5.

23. Deutsch, E.W., et al., The ProteomeXchange consortium in 2017: supporting the cultural change in proteomics public data deposition. Nucleic Acids Res, 2017. 45(D1): p. D1100-D1106.

24. Agarwal, V., et al., Predicting effective microRNA target sites in mammalian mRNAs. Elife, 2015. 4.

25. Dweep, H. and N. Gretz, miRWalk2.0: a comprehensive atlas of microRNA-target interactions. Nat Methods, 2015. 12(8): p. 697.

26. Hwang, Y.H., et al., Over-expression of c-raf-1 proto-oncogene in liver cirrhosis and hepatocellular carcinoma. Hepatol Res, 2004. 29(2): p. 113-121.

27. Boyault, S., et al., Transcriptome classification of HCC is related to gene alterations and to new therapeutic targets. Hepatology, 2007. 45(1): p. 42-52.

28. Indersie, E., et al., Tracking cellular and molecular changes in a species-specific manner during experimental tumor progression in vivo. Oncotarget, 2018. 9(22): p. 1614916162.

29. Chen, L., et al., Identification and bioinformatics analysis of miRNAs associated with human muscle invasive bladder cancer. Mol Med Rep, 2017. 16(6): p. 8709-8720.

30. Hironaka-Mitsuhashi, A., et al., A tissue microRNA signature that predicts the prognosis of breast cancer in young women. PLoS One, 2017. 12(11): p. e0187638.

31. Wu, X., et al., Identification and validation of potential biomarkers for the detection of dysregulated microRNA by qPCR in patients with colorectal adenocarcinoma. PLoS One, 2015. 10(3): p. e0120024.

32. Zhang, Q., et al., Domain-specific c-Myc ubiquitylation controls c-Myc transcriptional and apoptotic activity. Proc Natl Acad Sci U S A, 2013. 110(3): p. 978-83.

33. Czubaty, A. and A. Piekielko-Witkowska, Protein kinases that phosphorylate splicing factors: Roles in cancer development, progression and possible therapeutic options. Int J Biochem Cell Biol, 2017. 91(Pt B): p. 102-115.

34. Shen, J., et al., Epigenetic silencing of miR-490-3p reactivates the chromatin remodeler SMARCD1 to promote Helicobacter pylori-induced gastric carcinogenesis. Cancer Res, 2015. 75(4): p. 754-65.

35. Inoue, C., et al., SMARCD1 regulates senescence-associated lipid accumulation in hepatocytes. NPJ Aging Mech Dis, 2017. 3: p. 11.

36. Hooks, K.B., et al., New insights into diagnosis and therapeutic options for proliferative hepatoblastoma. Hepatology, 2017.

37. Leicht, D.T., et al., MEK-1 activates C-Raf through a Ras-independent mechanism. Biochim Biophys Acta, 2013. 1833(5): p. 976-86.

38. Karoulia, Z., E. Gavathiotis, and P.I. Poulikakos, New perspectives for targeting RAF kinase in human cancer. Nat Rev Cancer, 2017. 17(11): p. 676-691. 
1

2 1

2 3

4 5 6 7 8 9

39. Wang, F., et al., miR-195 is a key regulator of Raf1 in thyroid cancer. Onco Targets Ther, 2015. 8: p. 3021-8.

40. Liu, Z., et al., MiR-7-5p is frequently downregulated in glioblastoma microvasculature and inhibits vascular endothelial cell proliferation by targeting RAF1. Tumour Biol, 2014. 35(10): p. 10177-84.

41. Chen, L., et al., Expression and prognostic role of pan-Ras, Raf-1, pMEK1 and pERK1/2 in patients with hepatocellular carcinoma. Eur J Surg Oncol, 2011. 37(6): p. 513-20. 


\section{Figure legends}

2 FIG. 1. Identification of new targets of miR-4510 by proteomics.

3 Total proteins were extracted from Huh7 cells 24 hours after transfection with miR-4510 or a

4 CTRL, separated, digested, and analyzed by mass spectrometry. (a) Volcano plot shows $-\log 10 P$

5 value ( $y$ axis) versus fold change ( $x$ axis) of all analyzed proteins. Data were selected at a cutoff

6 value of adjusted- $P<0.05$. Overall protein analysis reported 322 down-regulated (Down), 289 up-

7 regulated (Up) and 2,688 unchanged proteins. Inset: Venn diagram shows overlaps between

8 down-regulated proteins (DR) and target predictions using TargetScan version 7.1 or miRWalk

9 version 3.0 algorithm. (b) Gene ontology analysis for the most significant hindered pathways upon

10 miR-4510 versus CTRL transfections in Huh7 cells. These pathways were identified by using

11 Ingenuity Pathway Analysis program ( $P$ value was calculated by Fisher's exact test). (c)

12 Assessment of proteomic data by western blot. Expressions of four different proteins were

13 analyzed following transfection of Huh7 cells. Lanes 1, 2, 3, and 4 illustrate expression levels of

14 CDK6, SKP2, CCNB1, and RAF1 proteins, respectively, and their down-regulation upon transfection

15 with miR-4510 as compared to CTRL. Immunodetections were quantified using ImageJ and

16 normalized to GAPDH signal. One representative blot is shown for each protein of interest. Bars

17 represent means \pm SD from four independent experiments in Huh7 cells (Mann-Whitney test, ${ }^{*} P$

18 < 0.05). Abbreviations: CCNB1, cyclin B1; CDK6, cyclin-dependent kinase 6; CTRL, small RNA

19 control; GAPDH, glyceraldehyde 3-phosphate dehydrogenase; miR-4510, mature microRNA 4510;

20 RAF1, RAF proto-oncogene serine/threonine-protein kinase; SD, standard deviation; SKP2, S-

21 phase kinase-associated protein 2. 
1 FIG. 2. RAF1 protein is increased in a majority of HCC and inversely correlates with miR-4510 in

2 biological samples.

3 (a-b) Relative expression of RAF1 protein measured by western blotting in 27 paired T and NTL

4 HCC samples (see raw data in Supporting Fig. S1). (a) Horizontal lines and whiskers in scatter dot-

5 plot represent mean \pm SD (Wilcoxon matched-pairs signed ranked test; ${ }^{* * *} p<0.001$ ). (b) Results

6 are presented as HCC/NTL expression ratios and show a distribution of samples into three

7 different populations: increased, unchanged, and decreased expression of RAF1 protein in HCC

8 compared to NTL. The median is shown as a full line, and the reference ratio value " 1 " is shown

9 as a dotted line. Decreased subgroup includes samples with an HCC/NTL ratio $<0.5$. Unchanged

10 subgroup includes samples with $\mathrm{HCC} / \mathrm{NTL}$ ratios between 0.5 and 1.5 , and increased subgroup

11 includes samples with an HCC/NTL ratio >1.5. (c) Representative immunostainings of RAF1 protein

12 in two paired T and NTL HCC samples. The brown-stained cells in the cytoplasm and the nucleus

13 were considered positive for RAF1 staining. The expression of RAF1 was higher in T as compared

14 to NTL for both patients (scale bars $=100 \mu \mathrm{m}$ ). (d) Relative expression of RAF1 mRNA in 112 HCC

15 and 21 NTL samples (ANOVA $P<0.0001$; Dunnett's multiple comparisons post-test; ${ }^{*} P<0.05,{ }^{* *} P$

16 0.01). Expression in HCC subgroups was as indicated in the main text. (e) Inverse correlation

17 between RAF1 protein expression measured by western blot and miR-4510 expression measured

18 by RT-PCR in 17 paired T and NTL HCC samples (Spearman $r$ correlation $=-0.4426 ; *^{*}<0.05$ ).

19 Abbreviations: ANOVA, analysis of variance; GAPDH, glyceraldehyde 3-phosphate

20 dehydrogenase; HCC, hepatocellular carcinoma; miR-4510, mature microRNA 4510; mRNA,

21 messenger RNA; NTL, nontumoral liver; RAF1, RAF proto-oncogene serine/threonine-protein

22 kinase; RT-PCR, real-time polymerase chain reaction; SD, standard deviation; T, tumor. 
1 FIG. 3. miR-4510 inactivates RAS/RAF/MEK/ERK pathway by directly targeting RAF1.

2 (a-d) Huh7 cells were transfected with miR-4510, siRAF1, or CTRL for 72 hours. Next,

3 RAS/RAF/MEK/ERK pathway was stimulated with EGF $(0.2 \mathrm{ng} / \mu \mathrm{L})$ for 10 minutes prior to total

4 protein or RNA extraction. (a-c) Relative expressions of RAF1 protein (a, lane 1); pERK1/2

$5(\operatorname{pERK1} 1 / 2)(a$, lane 2,); total $\operatorname{ERK} 1 / 2$ (a, lane 3); and two downstream effectors of

6 RAS/RAF/MEK/ERK pathway, (b) c-FOS and (c) c-MYC, were measured by western blot.

7 Immunosignals were quantified using ImageJ and normalized on Sypro Ruby staining of the (a)

8 membrane or on (b-c) GAPDH signal. The migration positions of molecular weight standards (in

$9 \mathrm{kDa}$ ) are indicated on the sides. One representative immunoblot is shown for each experiment.

10 Bars represent means \pm SD $\left(n=3\right.$; ANOVA <0.01; Dunnett's multiple comparisons post-test; ${ }^{*} P<$

$110.05, * * P<0.01, * * * P<0.001)$. (d) Expression level of RAF1 mRNA relative to 6 -Actin mRNA

12 (calculated as $2^{-\Delta \Delta C t}$ ) was assessed by RT-PCR 72 hours after transfection with miR-4510, siRAF1,

13 or CTRL. Bars represent mean \pm SD $(n=3$; ANOVA <0.001; Dunnett's multiple comparisons post-

14 test; $\left.{ }^{* * *} P<0.001\right)$. (e) Schematic representation of RAF1 3'- UTR:miR-4510 duplex based on

15 TargetScan prediction with miR-4510 site at position 219-225. Arrows indicate two point

16 mutations ( $r .221 \mathrm{C}>\mathrm{G}$ and r.223C $>\mathrm{G}$ ) in RAF1 3'-UTR. Huh7 cells expressing GFP transgene either

17 bearing WT or MUT RAF1 3'-UTR were transfected with miR-4510 or CTRL. The MFI ratio was

18 measured using the FunREG method as indicated in the main text. Bars represent means \pm SD ( $n$

$19=4 ;$ ANOVA <0.001; Bonferroni's multiple comparisons post-test; $\left.{ }^{* *} P<0.01\right)$. Abbreviations:

20 ANOVA, analysis of variance; CTRL, small RNA control; ERK, extracellular signal-regulated kinase;

21 EGF, epidermal growth factor; GFP, green fluorescent protein; MFI, mean fluorescence intensity;

22 miR-4510, mature microRNA 4510; mRNA, messenger RNA; MUT, mutated; pERK1/2, phospho- 
1 ERK1/2; RAF1, RAF proto-oncogene serine/threonine-protein kinase; RT-PCR, real-time

2 polymerase chain reaction; SD, standard deviation; siRAF1, small interfering RNA against RAF1;

3 UTR, untranslated region; WT, wild-type.

5 FIG. 4. RAF1 silencing impairs the tumoral behavior of HCC cells in vitro.

6 (a-c) Huh7 (left panels) and Hep3B (right panels) cells were transfected with CTRL, miR-4510, or

7 siRAF1, and the following experiments were performed: (a) Cellular growth was examined 6 days

8 after transfection. (b) Distribution of cell cycle (percentages of cells in $G_{0} / G_{1}, S$, and $G_{2} / M$ phases)

9 was analyzed 72 hours after transfection. (c) Senescent cells were quantified 8 days after

10 transfection. (d) Huh7 cells transfected with CTRL, miR-4510, or siRAF1 were grown to confluence,

11 and a wound was created using WoundMaker. Thus, cells were imaged with IncuCyte system

12 every 2 hours for 24 hours (only the 24-hour end point is shown). (a-d) Bars represent means \pm

13 SD ( $\mathrm{n}=3$; ANOVA $<0.01$; Dunnett's multiple comparisons post-test; ${ }^{*} P<0.05, * * P<0.01, * * * P<$

$140.001,{ }^{\#} P=0.0609$ ). (e) Huh7 (top panel) and Hep3B (bottom panel) cells stably expressing the

15 human RAF1 transgene lacking its 5'- and 3'-UTRs (RAF1) or an empty transgene (Empty) were

16 established by lentiviral transduction. Stable expressing cells were further transfected with CTRL

17 or miR-4510, and cellular growth was measured every 24 hours for 6 days using IncuCyte system.

18 Data are plotted as the percentage of confluence versus time \pm SD ( $n=3 ; 2$-way ANOVA $<0.001$;

19 Tuckey's multiple comparisons post-test; Empty/CTRL versus RAF1/CTRL: $* * P<0.01, * * * P<$

20 0.001; Empty/CTRL versus Empty/miR-4510 at 144hr: \#\#P<0.001; RAF1/CTRL versus RAF1/miR-

214510 at 144 hours: $\$ \$ p<0.001 ;$ Empty/miR-4510 versus RAF1/miR-4510: \& $P<0.05, \& \& P<0.01$, 
$1 \& \& \& P<0.001)$. Abbreviations: ANOVA, analysis of variance; CTRL, small RNA control; HCC,

2 hepatocellular carcinoma; miR-4510, mature microRNA 4510; mRNA, messenger RNA; RAF1, RAF

3 proto-oncogene serine/threonine-protein kinase; SD, standard deviation; siRAF1, small

4 interfering RNA against RAF1; UTR, untranslated region.

5

6 FIG. 5. RAF1 silencing inhibits HCC tumor development in vivo.

7 (a) Timeline illustrating the CAM assay. Twenty-four hours after transfection with miR-4510 or

8 CTRL, Huh7 cells were collected and grafted on the CAM at day 10 of embryonic development (=

9 day 0 of tumor age). Thus, tumor growth was monitored from day 11 to day 16 by

10 stereomicroscopy. Tumors aged of 6 days were extracted and fixed in formalin. (b) Lanes 1 and 2:

11 representative pictures of tumor having grown on CAM (top panels, scale bars $=3 \mathrm{~mm}$ ) and after

12 extraction and formalin-fixation (lane 2 panels, scale bars $=3 \mathrm{~mm}$ ) at day 16 . Lane 3 :

13 representative pictures of 6-day-aged tumors stained by HES (middle panels, scale bars $=100 \mu \mathrm{m}$ ).

14 Lanes 4 and 5: representative pictures of RAF1 (lane 4 panels) and Ki67 (bottom panels)

15 immunostainings in 6-day-aged tumors (scale bars $=50 \mu \mathrm{m}$ ). Magnifications were as indicated. (c-

16 d) On day 6 after miR-4510- or CTRL-transfected Huh7 cell implantation, (c) extracted tumor mass

17 was weighed, and (d) tumor thickness was measured with ImageJ using formalin-fixed paraffin-

18 embedded tissue sections. The total number of eggs analyzed per group is indicated in brackets

19 above the corresponding condition. Horizontal line and whiskers represent mean \pm SD (Mann-

20 Whitney test; ${ }^{* * * P}<0.001$ ). (e) Representative pictures of RAF1 immunostaining in tumors

21 deriving from Huh7 cells transfected with miR-4510 or CTRL (scale bars $=50 \mu \mathrm{m}$ ). (b and e, lanes 
14 and 5) The brown-stained cells were considered positive either for RAF1 or Ki67 protein.

2 Abbreviations: CAM, chick chorioallantoic membrane; CTRL, small RNA control; HCC,

3 hepatocellular carcinoma; HES, hematoxylin eosin saffron; miR-4510, mature microRNA 4510;

4 mRNA, messenger RNA; RAF1, RAF proto-oncogene serine/threonine-protein kinase; SD,

5 standard deviation.

6 


\section{Author contributions}

2 Amani Ghousein and Nicola Mosca performed the cellular and molecular analyses, the in vivo

3 analyses, and the histological staining. Justine Charpentier assisted in performing the cellular analyses.

$4 \quad$ Flora Cartier prepared transfected cells and protein extracts for proteomic analyses. Anne-Aurélie

5 Raymond and Jean-William Dupuy performed mass spectrometry and differential expression analysis.

6 Amani Ghousein, Anne-Aurélie Raymond, and Christophe F. Grosset performed bioinformatic and

7 statistical analyses. Paulette Bioulac-Sage provided tissue samples and clinical data, obtained written

8 informed consent and ethical approvals, and performed histological analysis. Amani Ghousein and

9 Christophe F. Grosset wrote the manuscript. Christophe F. Grosset supervised the work and obtained the

10 grants supporting this work. 
a

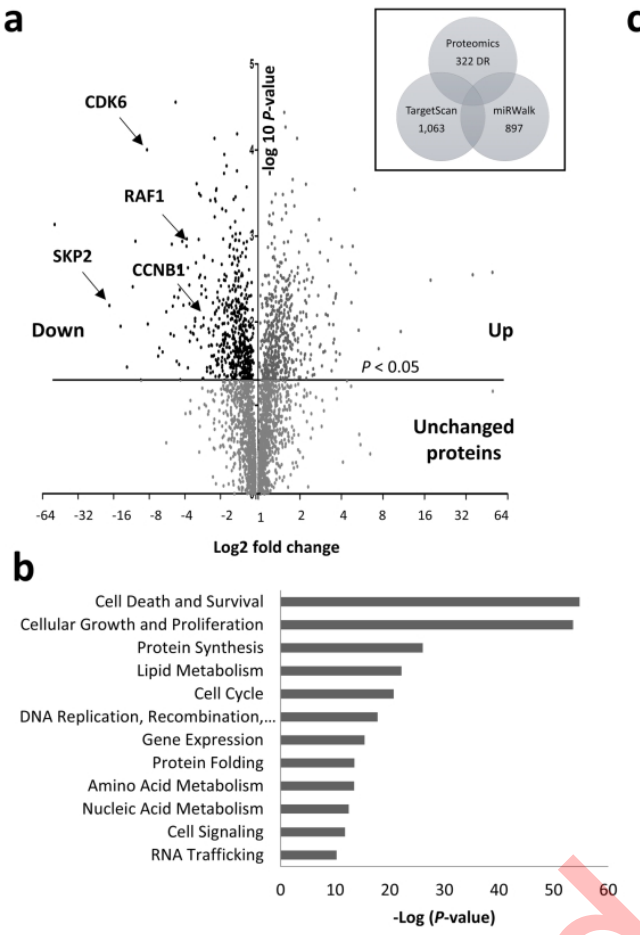

C
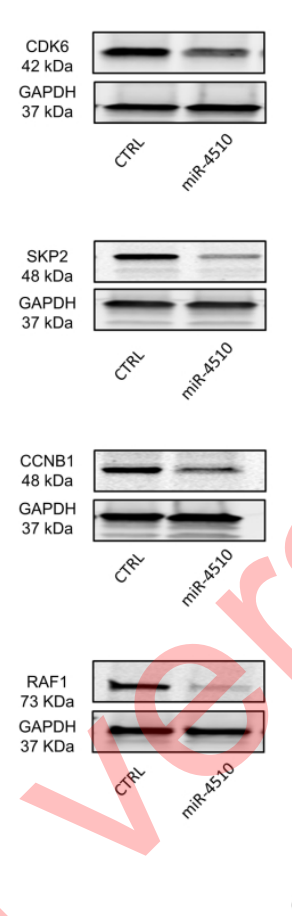

Ghousein A et al, Figure 1

Figure 1

$254 \times 190 \mathrm{~mm}(300 \times 300$ DPI $)$ 


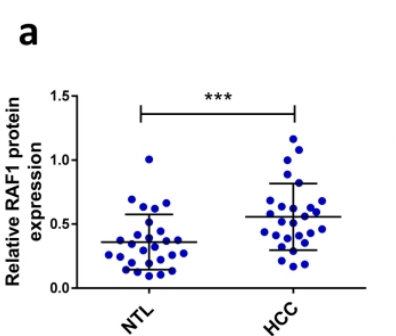

b

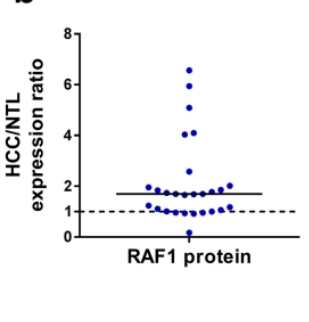

Patient 2

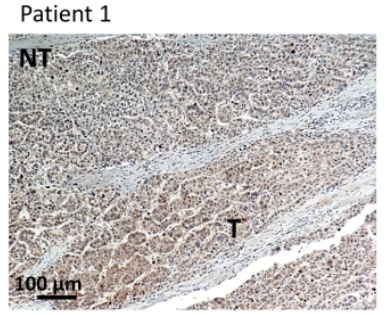

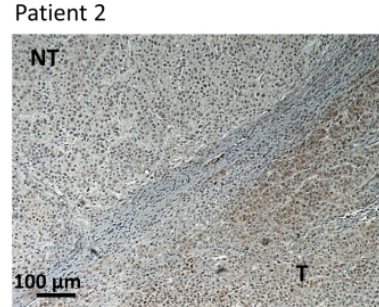

e

Ghousein A et al, Figure 2
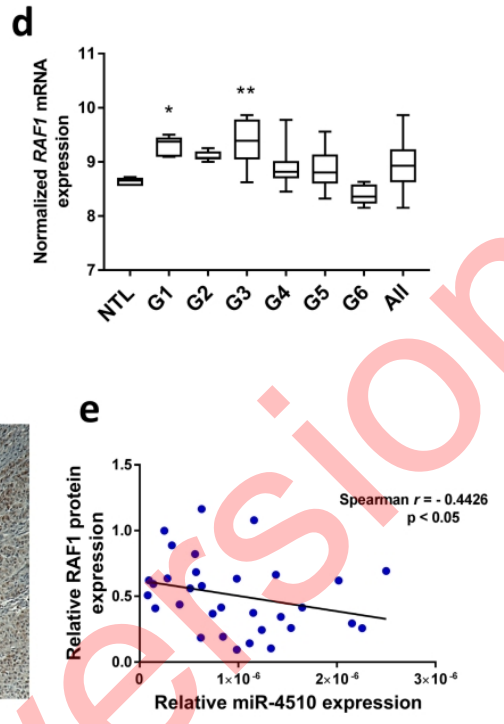

Figure 2

$254 \times 190 \mathrm{~mm}(300 \times 300$ DPI $)$ 
a

b
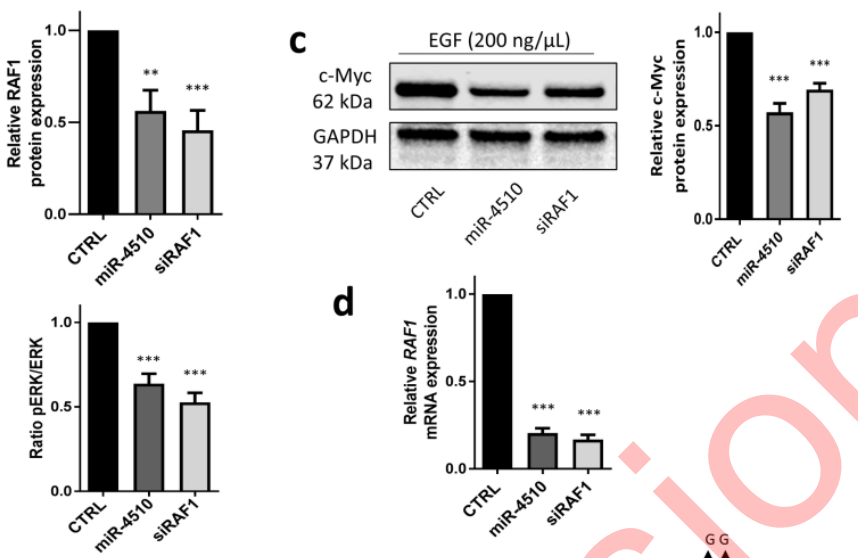

d

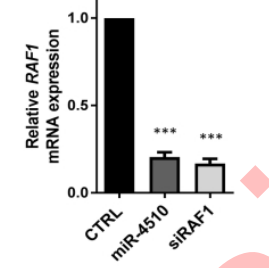

e RAF1 3'UTR 203 5'...UGCUGGUGUGUCCUGCUCCCUCC.... $3^{\prime} 226$

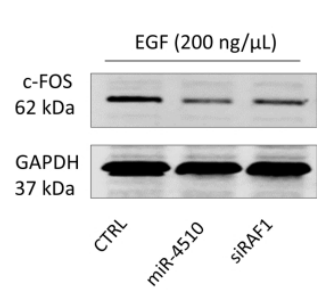

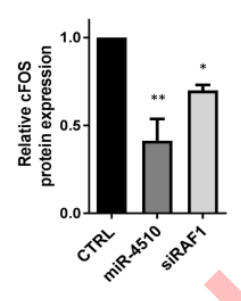

Ghousein A et al, Figure 3

Figure 3

$254 \times 190 \mathrm{~mm}(300 \times 300 \mathrm{DPI})$ 
b
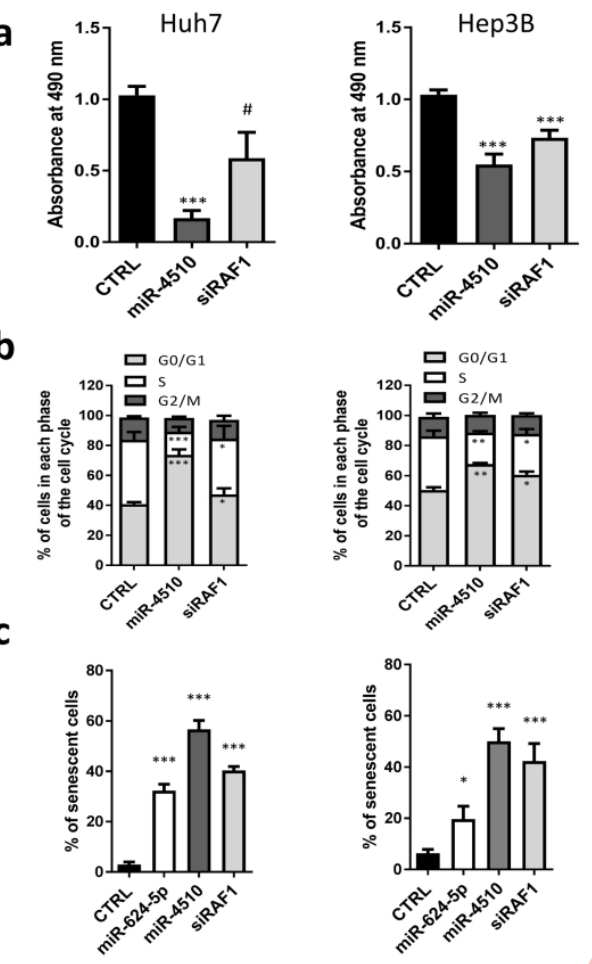

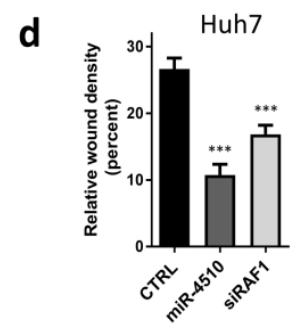

e
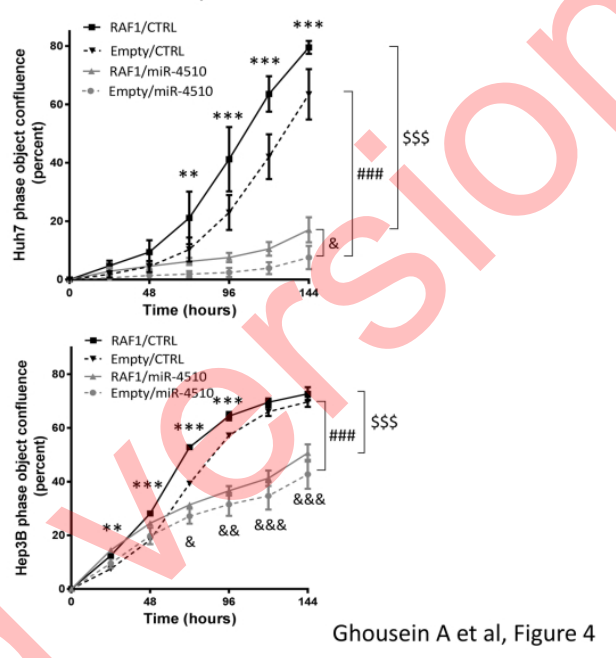

Figure 4

$254 \times 190 \mathrm{~mm}(300 \times 300 \mathrm{DPI})$ 

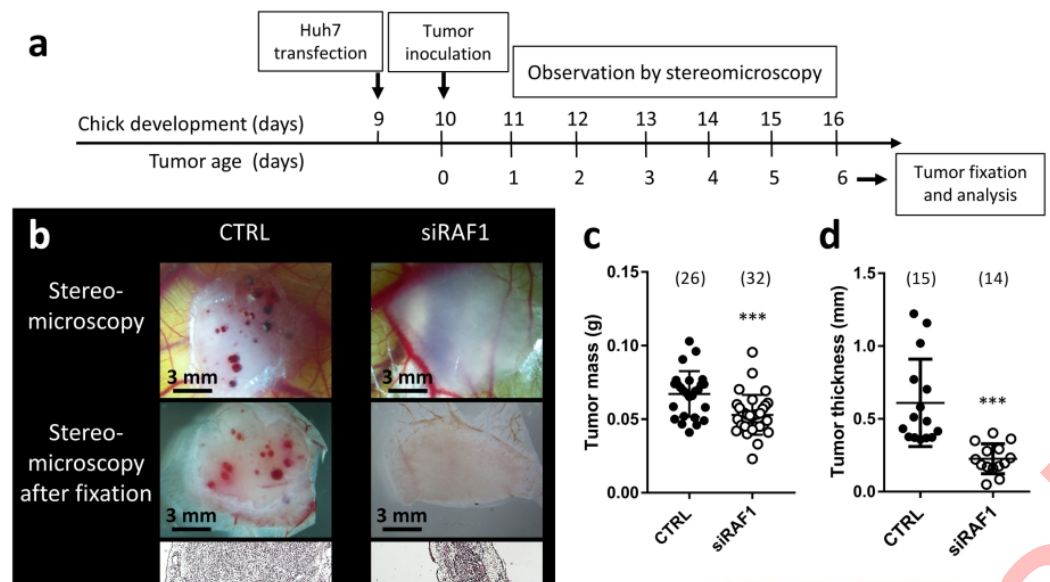

HES $10 \mathrm{x}$

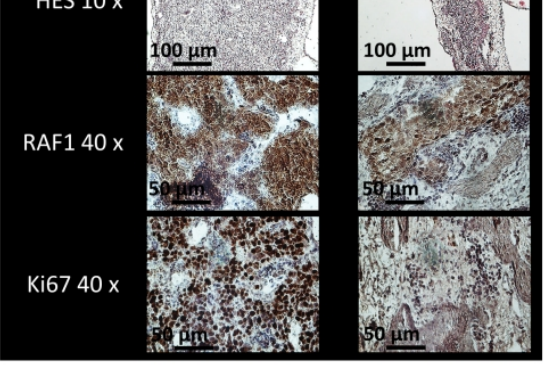

CTRL

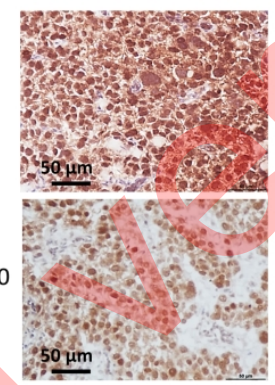

miR-4510

$050 \mu \mathrm{m}$

Ghousein A et al, Figure 5

Figure 5

$254 \times 190 \mathrm{~mm}(300 \times 300 \mathrm{DPI})$ 


\section{Supplementary Material}

\section{Plasmid constructions}

The lentiviral vectors pL-GFP-3'UTR-RAF1-WT and pL-GFP-3'UTR-RAF1-MUT were designed by inserting either the synthetic wild-type (WT) or mutated (MUT) (mutations r.221C>G and r.223C>G) RAF-1 3'-untranslated region (UTR) in the BsrGl site-digested pL-GFP plasmid. ${ }^{[1]}$ The NM-002880.3 sequence was used as a backbone for the sequencing of RAF1 3'-UTR into the pEX-A2 insert-donor plasmid (Eurofins Genomics, Germany).

For the pL-RAF1 vector, the open reading frame (ORF) of RAF1 was amplified from pLX304 vector (ORF ccsbBroad304_06837, Dharmacon, Inc., Lafayette, CO) using the forward primer GCGGATCCACCATGGAGCACATACAGGGAGC (underlined nucleotides represent the BamHI restriction site) and the reverse primer GCGCTAGCTTAGTAGACAGGCAGCCTCGG (underlined nucleotides represent the Nhel restriction site). The obtained polymerase chain reaction (PCR) product was subcloned into an intermediary TA vector (TA Cloning Kit, Invitrogen, Carlsbad, CA, USA). Next, the obtained plasmid was cut with BamHI and $\mathrm{NHel}$, and the insert containing RAF1 ORF was purified. In parallel, pL-GFP was cut with the same restriction enzymes to remove Green Fluorescent Protein (GFP) coding region where RAF1 ORF was ligated instead, thus yielding the PL-RAF1 vector that was used to produce infectious lentiviral particles.

\section{RNA isolation and quantitative real-time PCR}

Total RNA was extracted from cultured cells using Trizol (Sigma, St Louis, MO, USA) reagent or mirVana miRNA Isolation Kit (Life Technologies, Carlsbad, CA, USA) according to the manufacturer's instructions. miR-4510 expression was measured as described previously [2]. To quantify mRNA expression, complementary DNA was synthesized with the Maxima Reverse Transcriptase kit (Thermo Scientific, Waltham, MA, USA). Quantitative PCR (qPCR) amplifications were performed in $12-\mu \mathrm{L}$ multiplex PCR reactions containing 1X SYBR Green Supermix (Quanta Biosciences, Beverly, MA, USA) 
on a C1000 Thermal Cycler (Bio-Rad, Hercules, CA, USA). Beta-actin was used as internal control. The relative mRNA levels of gene expression were calculated using the $2^{-\Delta \Delta C t}$ method. The primer sequences were RAF1 forward (5' GGAGACACATGGGATTTTGG 3') and reverse (5' GCTGTGAAAGGAGGACGTGT 3') and beta-actin forward (5' TCCCTGGAGAAGAGCTACGA 3') and reverse (5' AGCACTGTGTTGGCGTACAG 3'), all purchased from Eurofin Genomics. Primer efficiency was between 95\% and 105\%. miR-4510 expression was measured as described before [2].

\section{Cell viability assay}

A total of 3,000 cells/well were seeded in $96-$ well plates, and cell proliferation was evaluated by using tetrazolium compound-based CellTiter 96 AQueous One Solution Cell Proliferation (3-(4,5dimethylthiazol-2-yl)-5-(3-carboxymethoxyphenyl)-2-(4-sulfophenyl)-2H-tetrazolium [MTS]) assay (Promega, Madison, WI, USA), according to the manufacturer's instructions. Then, $20 \mu \mathrm{L}$ of the MTS reagent solution was added to each well and incubated at $37^{\circ} \mathrm{C}$ in $5 \% \mathrm{CO}_{2}$ for 2 hours. Cell proliferation was assessed at days 1 and 6 after transfection. Absorbance was read at $490 \mathrm{~nm}$ using a ClarioStar (BMG Labtech, Ortenberg, Allemagne).

\section{Cell cycle}

Cell cycle analysis was performed using the APC BrdU flow kit (BD Pharmingen, San Diego, CA) according to the manufacturer's instructions. Briefly, 200,000 cells were seeded into a 6 -well plate with volume $2 \mathrm{~mL}$ and then transfected. After 3 days, BrdU was added into the cell culture medium for 45 minutes. The incorporated BrdU was detected using an APC anti-BrdU fluorescent antibody, and the levels of cell-associated BrdU were measured by flow cytometry using a fluorescence-activated cell sorting (FACS) CANTO II (BD Biosciences, San Jose, CA, USA). 


\section{Cell death assays}

Prior to apoptosis detection, 200,000 cells were seeded into a 6-well plate in a volume of $2 \mathrm{~mL}$ and transfected. Three days later, total cells were collected, and apoptosis was analyzed using the Annexin V-PE/7-Amino-Actinomycin (AAD) apoptosis detection kit (BD Pharmingen, San Diego, $C A$ ). Viable cells with intact membranes exclude 7-ADD and are PE Annexin V negative. Fluorescence generated by the cell-bound Annexin V-PE, which measures the percentage of early apoptotic cells, and the 7AAD, which measures the percentage of late apoptotic cells, were analyzed by FACS. Total apoptosis was calculated by adding the percentage of late apoptotic cells (Annexin V-PE ${ }^{\text {High }} / 7$ ADD $^{\text {High }}$ )

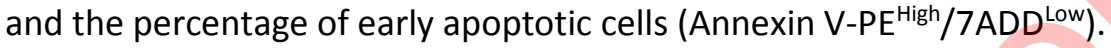

\section{Cell senescence assay}

Cell senescence was assessed using the Senescence Beta-Galactosidase Staining kit (Cell Signaling, Danvers, MA, USA) according to manufacturer's instructions. Cells were plated in a 24-well plate at a density of 5,000 cells/well in a volume of $500 \mu \mathrm{L}$ and transfected by either CTRL, miR-4510, siRAF1, or miR-624-5p. After 4 days, cells were trypsinized and seeded onto a cover slip 24-well plate where they were subjected to second transfection. Four days later, cells were fixed, and betagalactosidase activity was revealed by the addition of X-gal and staining solution. Cells were counterstained by eosin $0.5 \%$ and observed under a microscope. Only blue cells were counted as a measure of senescence.

\section{Wound healing and migration assays}

Huh7 cells were transfected with miR-4510, siRAF1, or CTRL. After 24 hours, cells were trypsinized and moved into 96-well plates where they were seeded at a density of 15,000 cells. Six hours later, a wound 
was created using WoundMaker, and cells were imaged with IncuCyte (Essen BioSciences, USA) every 2 hours for 24 hours. To obtained more homogeneous layers of Huh7 cells, especially after transfection with the pro-apoptotic and pro-senescent miR-4510, two-well cell culture inserts (Biovalley, France) were used. Huh7 cells were transfected with miR-4510 or CTRL. After 48hours, cells were trypsinized and moved into 2-well culture inserts at a density of 50,000 cells per well. Eight-teen hours later, empty-space colonization by cells was imaged at 8,24 and 48 hours with an InCellis microscope (Bertin Technologies, France) and quantifications were performed with Image J.

\section{FunREG and DF-FunREG screening}

FunREG and DF-FunREG analyses were performed 3 days after transfection as described $[1,3]$ with minor modifications, including fluorescence measurements using CLARIOstar multiplate reader (BMG Labtech, Ortenberg, Allemagne).

\section{Immunohistochemistry}

The 3.5- $\mu \mathrm{m}$-thick sections were de-paraffinized and rehydrated, and antigen retrieval was performed in a citrate buffer $\mathrm{pH} 9$ solution. All staining procedures were performed by an autostainer (DakoAgilent Clara, USA) using standard reagents provided by the manufacturer. The sections were incubated with rabbit anti-RAF-1 (1:250, ab154754, Abcam) or mouse anti-Ki67 (1:100, M7240, Dako). Incubation in horseradish peroxidase (EnVision Flex/HRP, Dako-Agilent) or secondary AB for 20 minutes was used for signal amplification. 3,3'-diamino-benzidine (DAB, Dako) development was used for detecting primary antibodies. The slides were counterstained with hematoxylin, dehydrated, and mounted. Each immunohistochemical run contained a negative control (buffer, no primary antibody). Sections were visualized with a Nikon-Eclipse501 microscope, and images were acquired using NISElements F. 


\section{References}

1. Maurel, M., et al., A functional screening identifies five micrornas controlling glypican-3: role of mir-1271 down-regulation in hepatocellular carcinoma. Hepatology, 2013. 57(1): p. 195204.

2. Cartier, F., et al., New tumor suppressor microRNAs target glypican-3 in human liver cancer. Oncotarget, 2017. 8(25): p. 41211-41226.

3. Indersie, E., et al., MicroRNA therapy inhibits hepatoblastoma growth in vivo by targeting beta-catenin and Wnt signaling. Hepatol Commun, 2017. 1(2): p. 168-183. 


\section{Supplementary Figures}

Supplementary FIG. S1. Expression of RAF1 protein in tumoral and nontumoral tissues from HCC patients. Total proteins were extracted from 27 paired T and adjacent NTL HCC samples and 2 normal liver specimens from the same patient (NL1) that were used as controls. Thus, RAF1 protein level was quantified by western blotting in P samples using Huh7 cell protein extract as internal C. Immnunodetections were normalized on Sypro Ruby staining of the membranes and on C. Abbreviations: C, calibrator; NL1, normal liver; NTL, nontumoral liver; P, patient; T, tumor.

Supplementary FIG. S2. Immunostaining of RAF1 protein in tumoral and nontumoral tissues from HCC patients. (a) HES staining of sectioned tissues (scale bars $=100 \mu \mathrm{m}$ ). (b) RAF1 immunostaining of sectioned tissues. Pictures show difference in expression between the tumoral and nontumoral parts of the liver (scale bars $=100 \mu \mathrm{m}$ ). (c) A normal liver tissue was sectioned at $3.5 \mu \mathrm{m}$ thickness. The left panel shows HES staining, and the right panel shows RAF1 immunostaining (scale bars $=100 \mu \mathrm{m}$ ). RAF1 staining is negative in normal liver. Abbreviation: HES, hematoxylin eosin saffron.

Supplementary FIG. S3. miR-4510 or siRAF1 inhibits RAF1 protein expression in transfected Hep3B cells. Expression levels of RAF1 protein assessed by western blot on Hep3B whole cell extracts 72 hours after transfection with miR-4510, siRAF1, or CTRL. Immnunodetections were normalized on GAPDH signal. The migration positions of molecular weight standards (in kDa) are 
indicated on the sides. One representative immunoblot is shown. Bars represent means \pm SD (n $=3$; ANOVA <0.001; Dunnett's multiple comparisons post-test; $\left.{ }^{* * *} P<0.001\right)$. Abbreviations: ANOVA, analysis of variance; CTRL, small RNA control; HES, hematoxylin eosin saffron; SD, standard deviation; siRAF1, small interfering RNA against RAF1.

Supplementary FIG. S4. miR-4510 or RAF1 silencing inhibits HCC cell proliferation. (a-b) Representative data of APC BrdU/7-AAD measurements performed by flow cytometry in (a) Huh7 cells and (b) Hep3B cells transfected with miR-4510, siRAF1, or CTRL.

Supplementary FIG. S5. miR-4510 or RAF1 silencing induces senescence of HCC cells. (a-b) Huh7 and Hep3B cells were transfected with the indicated small RNAs. Eight days later, cells were stained for beta-galactosidase activity and counterstained with eosin. Blue cells were considered positive. Representative pictures (scale bars $=100 \mu \mathrm{m}$ ) of (a) X-gal-positive Huh7 and (b) Hep3B cells.

Supplementary FIG. S6. miR-4510 induces apoptosis, but RAF1 silencing has limited proapoptotic ability. (a-b) Representative data of Annexin V/7AAD staining measured by flow cytometry in (a) Huh7 cells and (b) Hep3B transfected with miR-4510, siRAF1, or CTRL. For each small RNA, the left graphs represent the cell population selected to analyze apoptosis in the right graphs. Q1: early apoptotic cells; Q2: late apoptotic cells; Q3: viable cells; Q4: nonviable cells. As 
shown in the top right panel, total apoptosis was calculated by adding the percentage of late apoptotic cells (Annexin V-PE ${ }^{\text {High }} / 7 A D D^{\text {High }}$ ) and the percentage of early apoptotic cells (Annexin V-PE $\left.{ }^{\text {High }} / 7 A D D^{\text {Low }}\right)$. Bars represent means $\pm S D(n=3$; ANOVA <0.05; Dunnett's multiple comparisons post-test; $* P<0.05, * * * P<0.001)$.

Supplementary FIG. S7. Combined apoptotic effect of miR-4510 and Sorafenib on HCC cells. (a) Two days after transfection by miR-4510 or control RNA (Ctrl), Huh7 cells were treated with $10 \mu \mathrm{M}$ sorafenib for 24 hours. Thus, the percentage of apoptotic cells was measured ( $n=3$, ANOVA $p<0.001$; Bonferroni's multiple comparisons test: $* p<0.05$; $* * * p<0.001)$. (b-c) Treatment of Huh7 cells by $10 \mu \mathrm{M}$ sorafenib strongly decreased RAF1 protein expression (b) and induced miR4510 expression. (b) Immnunoblots showing RAF1 and GAPDH signals. The migration position of the proteins compared to the molecular weight standards is as indicated in kDa. Immunoblots of three independent experiments are shown. (c) Relative expression of miR-4510 in Huh7 cells treated or not by Sorafenib (Soraf). Bars represent means \pm SD ( $n=6$; two-tailed Wilcoxon matched-pairs signed rank test; $* \mathrm{P}<0.05)$.

Supplementary FIG. S8. miR-4510 or RAF1 silencing inhibits migration of Huh7 cells. (a) Representative picture of migrating Huh7 cells 24 hours after wound creation. Huh7 cells were transfected with the indicated small RNAs and grown to confluence. Thus, a wound was created ( $T=0$ hours), and the plate was placed in the IncuCyte system. Migration was monitored by taking pictures every 2 hours for 24 hours (24-hour end point is shown). (b) Left panel: Representative 
picture of migrating Huh7 cells at $0,8,24$ and 48 hours after removal of the 2-well culture insert. Right panel: Percentage of area closure using Huh7 cells transfected with Control RNA (CTRL) or miR-4510. Bars represent means \pm SD ( $n=3$, two-way ANOVA $p<0.001$; Sidak's multiple comparisons test: $* * * p<0.001)$.

Supplementary FIG. S9. Ectopic expression of RAF1 protein in Huh7 and Hep3B cells following lentiviral transduction. Huh7 and Hep3B cells were transduced at an $\mathrm{MOI}$ of 5 with lentivirus either harboring the RAF1 transgene lacking its 3'- and 5'-UTRs or an empty transgene. Then, 24 hours after transduction, cells were washed and amplified. Next, total proteins were extracted from each cell line, and the expression of RAF1 protein was examined by western blot. Immnunodetections were normalized on Sypro Ruby staining of the membrane. The migration positions of molecular weight standards (in $\mathrm{kDa}$ ) are indicated on the sides. One representative immunoblot is shown. Bars represent mean $\pm S D\left(n=3\right.$; Mann-Whitney test; $\left.{ }^{*} P<0.05\right)$. Abbreviation: $\mathrm{MOI}$, multiplicity of infection.

Supplementary FIG. S10. Effect of miR-4510 transfection on RAF1 protein level in Huh7 cells ectopically expressing RAF1 transgene or an empty transgene. Huh7 cells stably expressing RAF1 transgene or an empty (Empty) transgene were transfected with miR-4510 or CTRL. Then, 72 hours after transfection, cells were lysed, and total proteins were extracted. Relative level of RAF1 protein expression was quantified by western blot. Immnunodetections were normalized on GAPDH signal. The migration positions of molecular weight standards (in kDa) are indicated 
on the sides. One representative immunoblot is shown. Bars represent means $\pm S D(n=3$; ANOVA $<0.001$; Dunnett's multiple comparisons post-test; $* P<0.05, * * * P<0.001)$.

Supplementary FIG. S11. RAF1 silencing impairs HCC growth in vivo. (a-b) HES staining of Huh7 cell-derived tumors grown on the CAM after transfection with (a) CTRL or (b) siRAF1 (scale bars $=100 \mu \mathrm{m}$ ). (c-d) RAF1 staining of Huh7 cell-derived tumors grown on the CAM after transfection with (c) CTRL or (d) siRAF1 (scale bars $=50 \mu \mathrm{m}$ ). One representative blank tissue section is shown. (e-f) Ki67 staining of Huh7 cell-derived tumors grown on the CAM after transfection with (e) CTRL or (f) siRAF1 (scale bars $=50 \mu \mathrm{m}$ ). One representative blank tissue section is shown. Brownstained cells were considered positive.

Supplementary FIG. S12. miR-4510 decreases RAF1 expression in vivo. (a-b) HES staining of Huh7 cell-derived tumors grown on the CAM after transfection with (a) CTRL or (b) miR-4510 (scale bars $=100 \mu \mathrm{m}$ ). (c-d) RAF1 staining of Huh7 cell-derived tumors grown on the CAM after transfection with (c) CTRL or (d) miR-4510 (scale bars $=50 \mu \mathrm{m}$ ). One representative blank tissue section is shown. Brown-stained cells were considered positive.

Supplementary FIG. S13. RAF1 5'-UTR contains two predicted miR-4510 sites. Schematic representation of RAF1 untranslated regions (UTR):miR-4510 duplexes based on predictions with 
RNA Hybrid for RAF1 5'-UTR and TargetScan for 3'-UTR (ref: NM_002880.3, Homosapiens Raf-1 proto-oncogene, serine/threonine protein kinase, mRNA). 
Supplementary Table S1: Clinical and genetic features of 27 paired tumor and nontumoral liver HCC samples of our cohort.

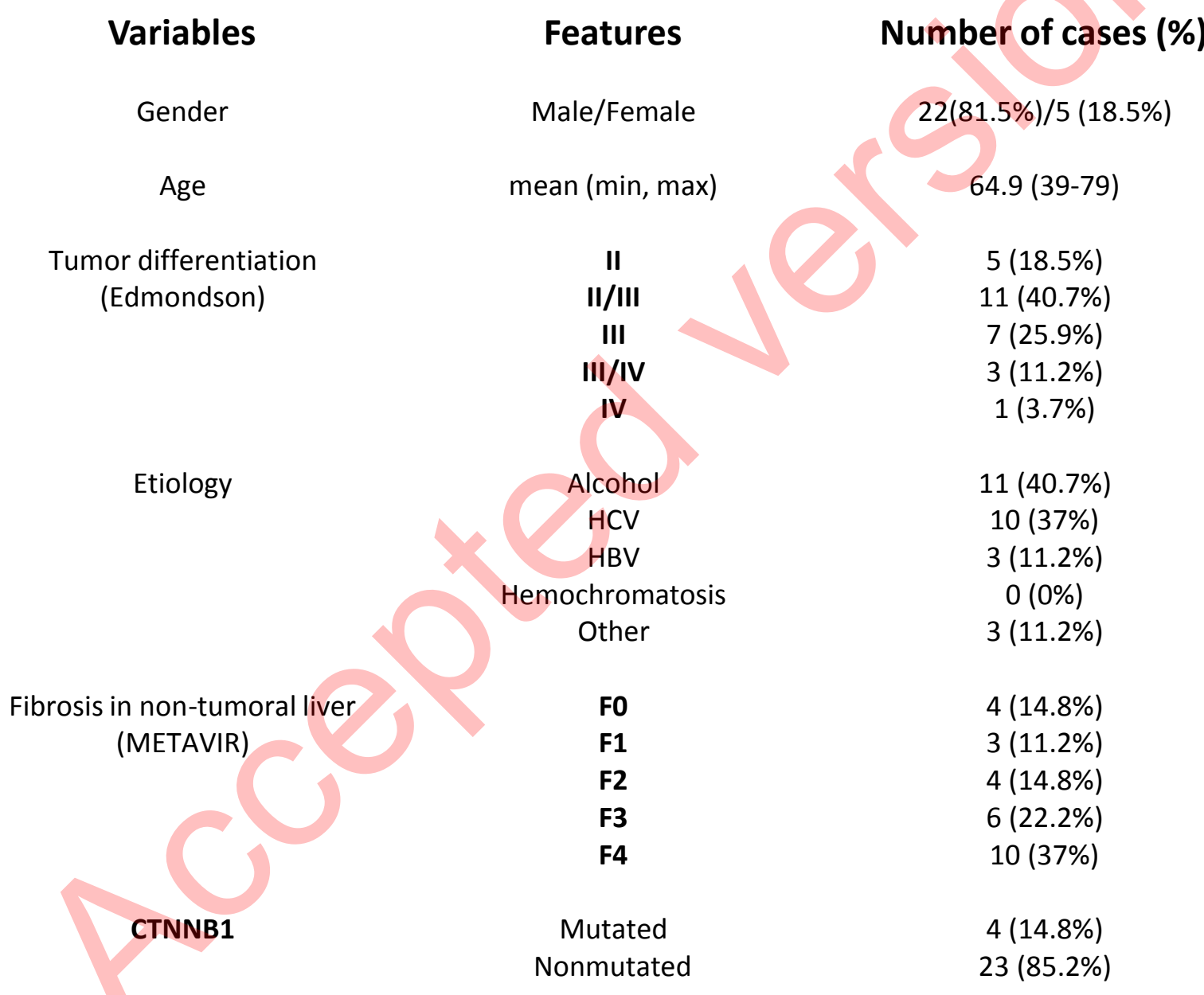


Supplementary Table S4: Cellular and molecular processes and genes deregulated in miR-4510-transfected Huh7 cells.

\begin{tabular}{|c|c|}
\hline Categories & Genes \\
\hline $\begin{array}{l}\text { Cellular Growth and } \\
\text { Proliferation }\end{array}$ & $\begin{array}{c}\text { RAF1, COL14A1, MYH9, IGF2BP3, } \\
\text { CCL20, SOD2 }\end{array}$ \\
\hline Protein Synthesis & $\begin{array}{c}\text { RAF1, APOB, MYH9, IGF2BP3, NCSTN, } \\
\text { ARHGEF1 }\end{array}$ \\
\hline Cell Cycle & $\begin{array}{c}\text { RAF1, MYH9, ANKRD17, NCAPG, SOD2, } \\
\text { EZR }\end{array}$ \\
\hline $\begin{array}{l}\text { DNA Replication, } \\
\text { Recombination, and Repair }\end{array}$ & $\begin{array}{c}\text { RAF1, MCM6, SRPK1, NCAPG, CUL4A, } \\
\text { SART1, PBK, SOD2 }\end{array}$ \\
\hline Gene Expression & $\begin{array}{l}\text { RAF1, JAK1, CUL4A, RPS6KA3, PPP4C, } \\
\text { SMARCA4 }\end{array}$ \\
\hline
\end{tabular}


C $\frac{\mathrm{P} 1}{\mathrm{~T} N \mathrm{NT}} \quad \frac{\mathrm{P} 2}{\mathrm{~T} N \mathrm{NT}} \quad \frac{\mathrm{P} 3}{\mathrm{~T} N \mathrm{NT}} \quad \frac{\mathrm{P} 4}{\mathrm{~T} N \mathrm{NT}}$
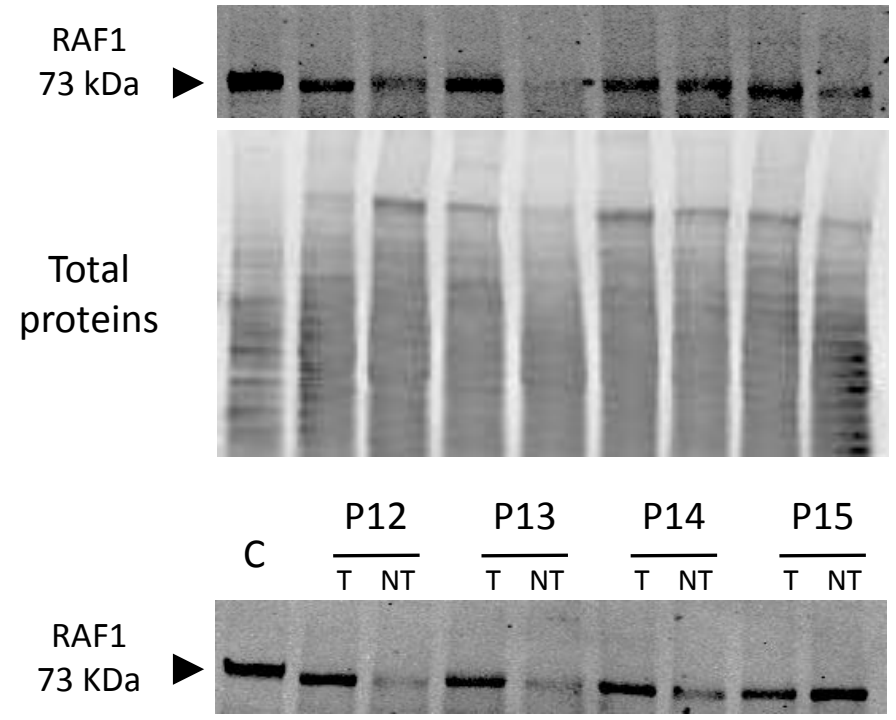$$
\text { . }
$$

Total proteins

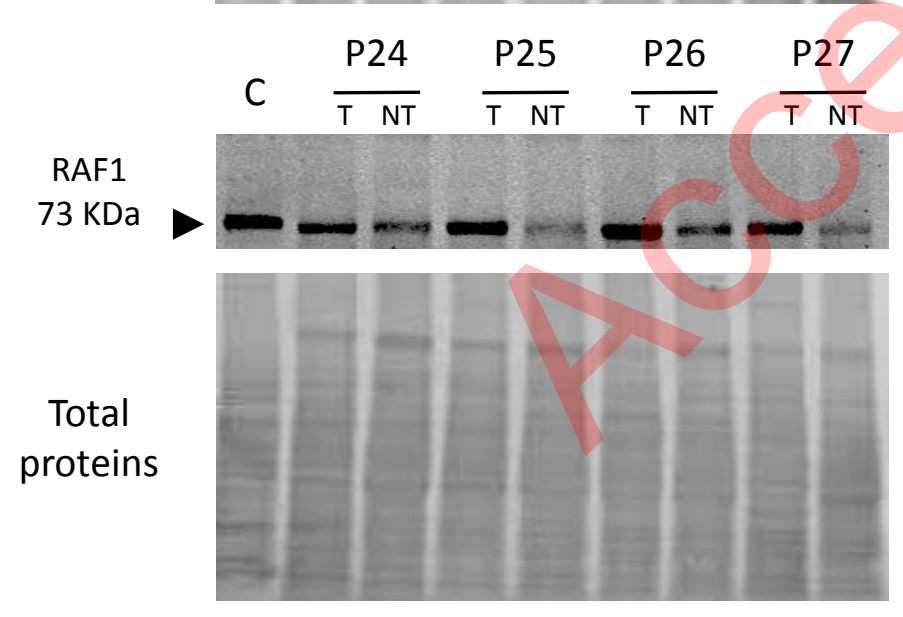

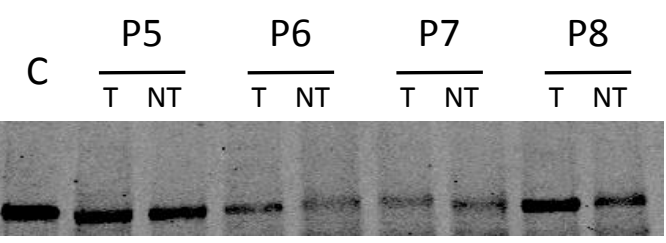

C $\frac{\mathrm{NL1}}{\mathrm{NT} N \mathrm{NT}} \quad \frac{\mathrm{P} 9}{\mathrm{~T} N \mathrm{NT}} \quad \frac{\mathrm{P} 10}{\mathrm{~T} N \mathrm{NT}} \quad \frac{\mathrm{P} 11}{\mathrm{~T} N \mathrm{NT}}$
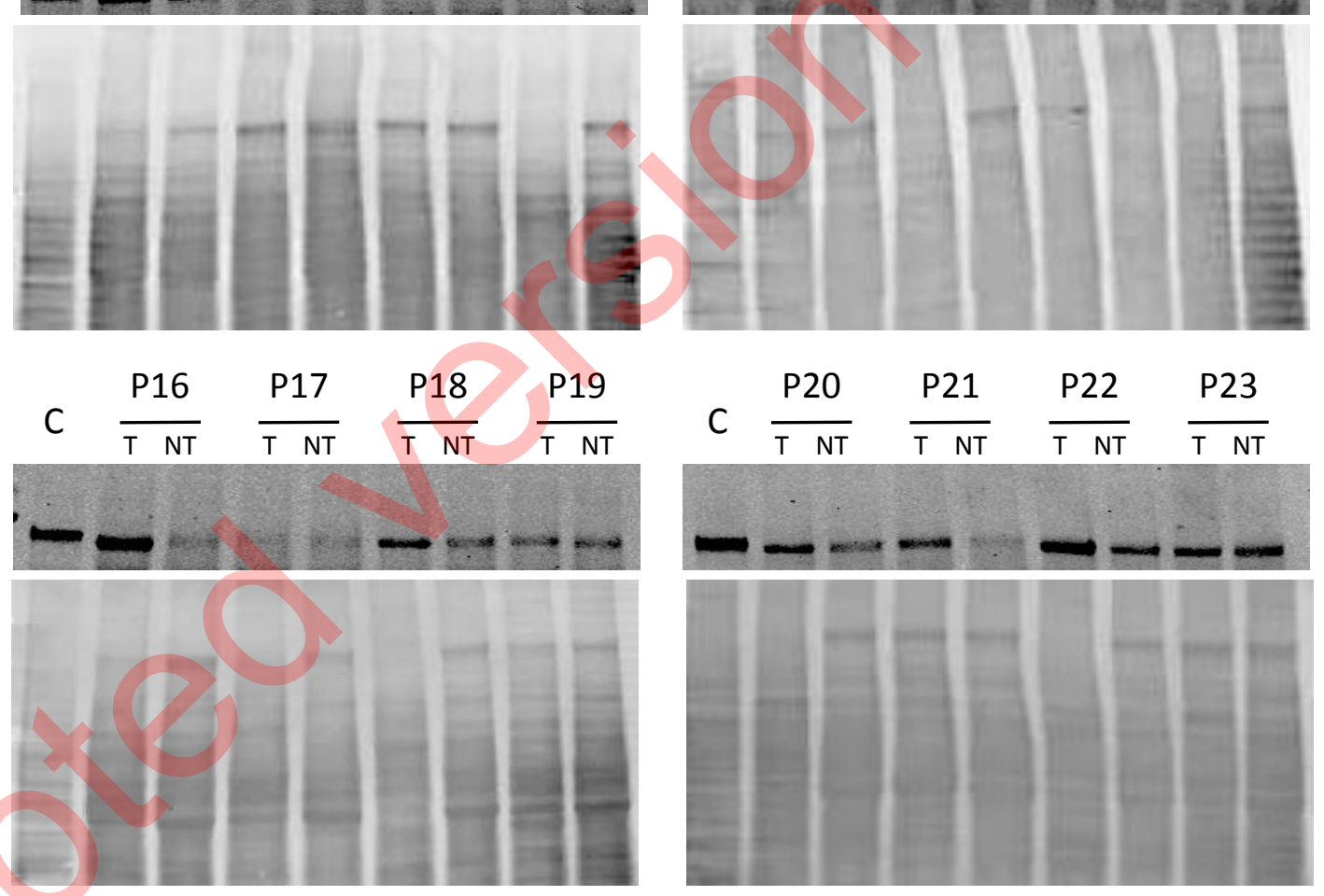

\section{-}



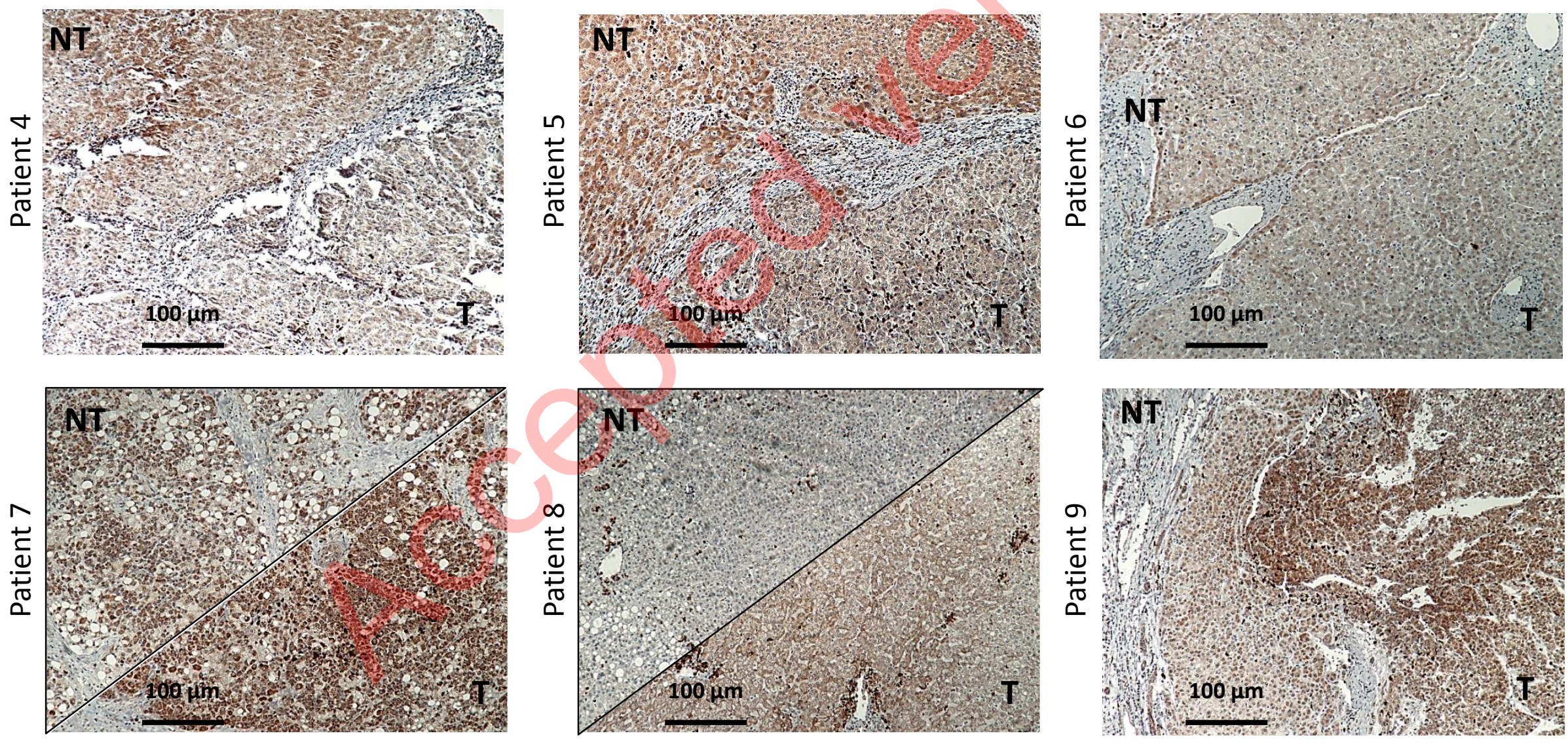

Ghousein A et al, Supplementary Figure S2 


\section{C}

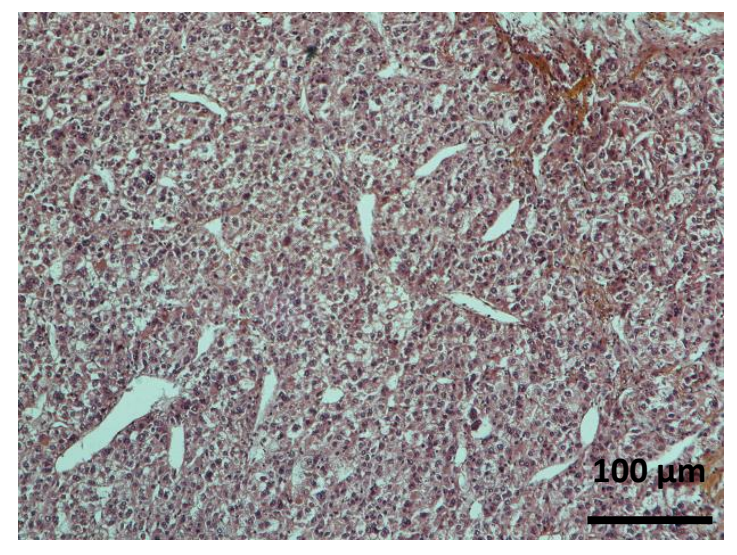

HES

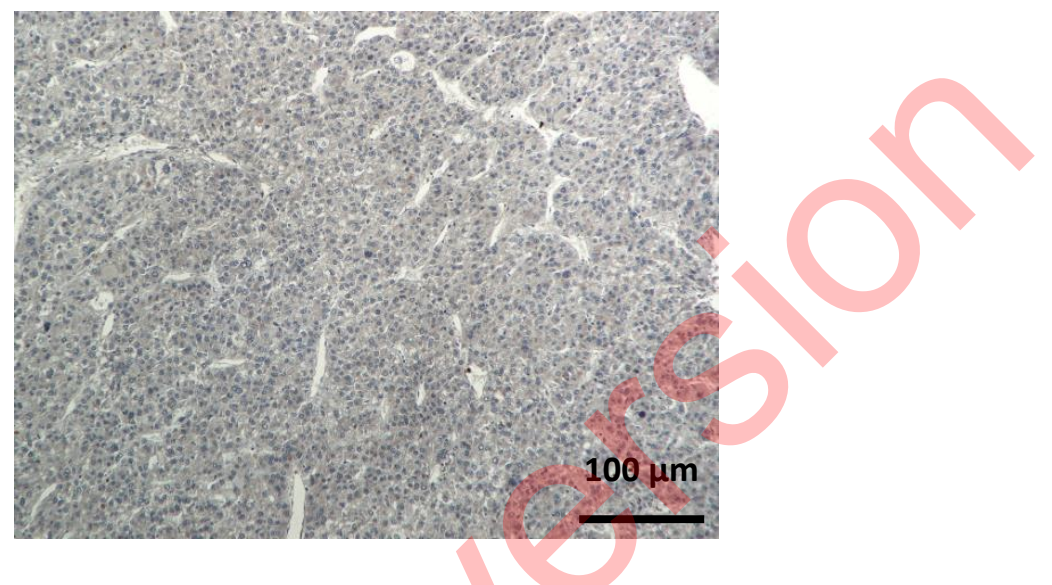

RAF1

immunostaining

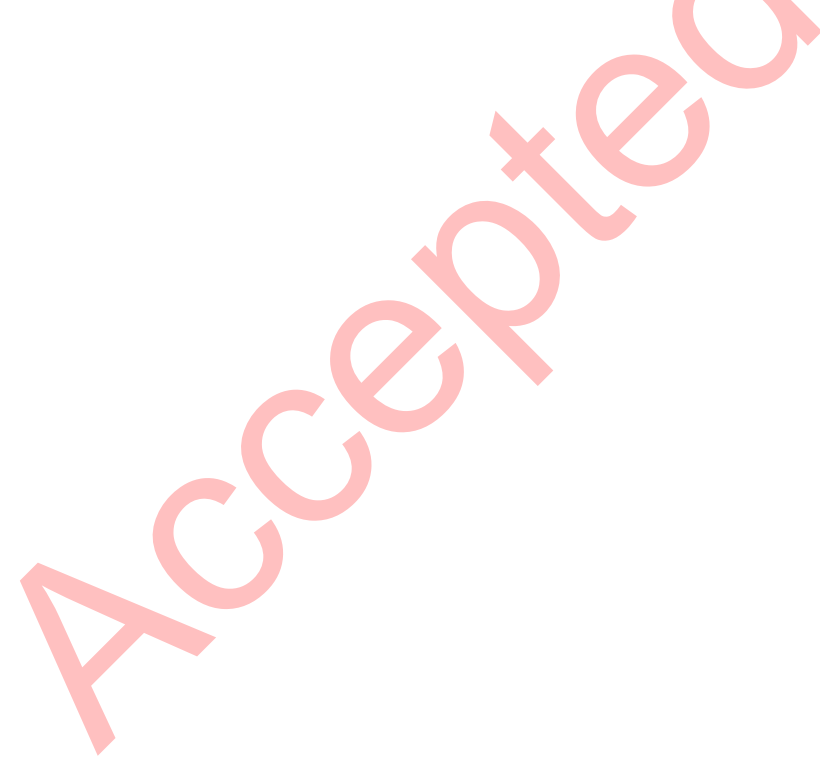

Ghousein A et al, Supplementary Figure S2 
Hep3B cells
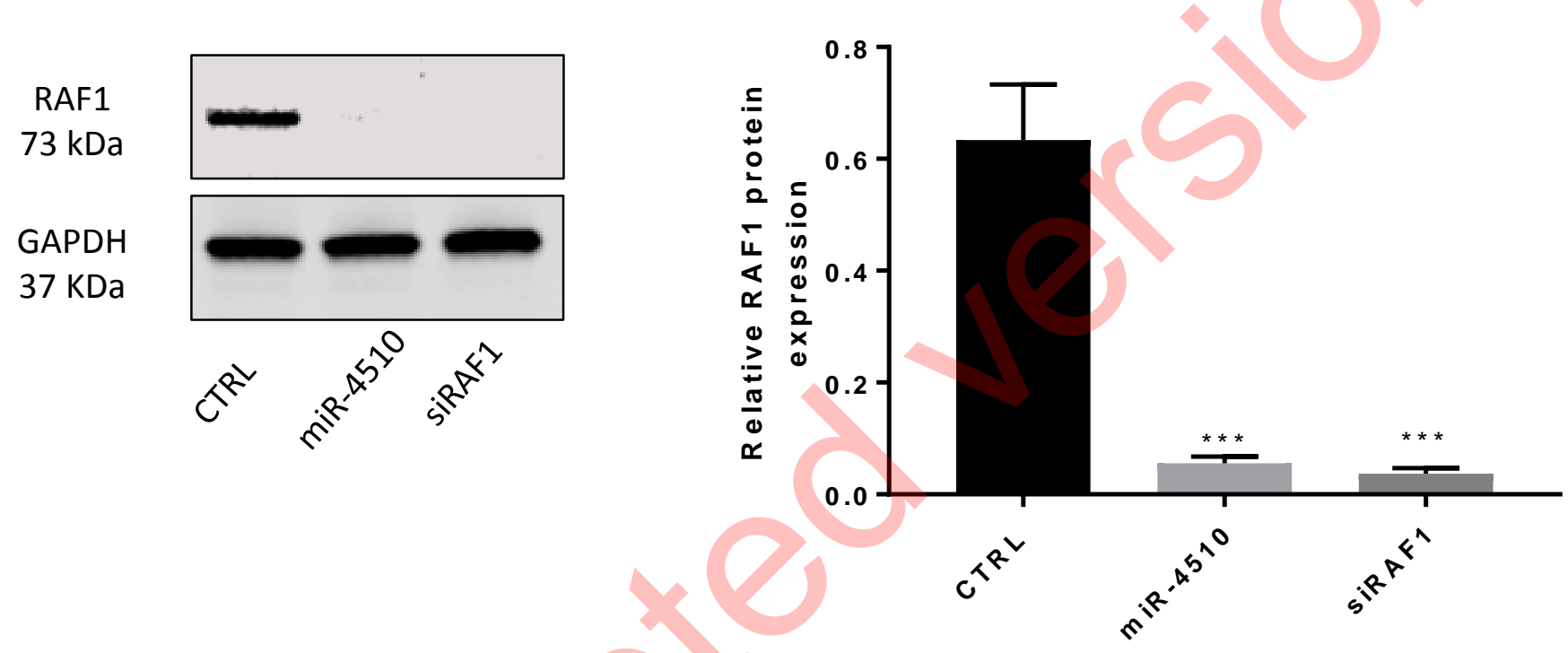

Ghousein A et al, Supplementary Figure S3 

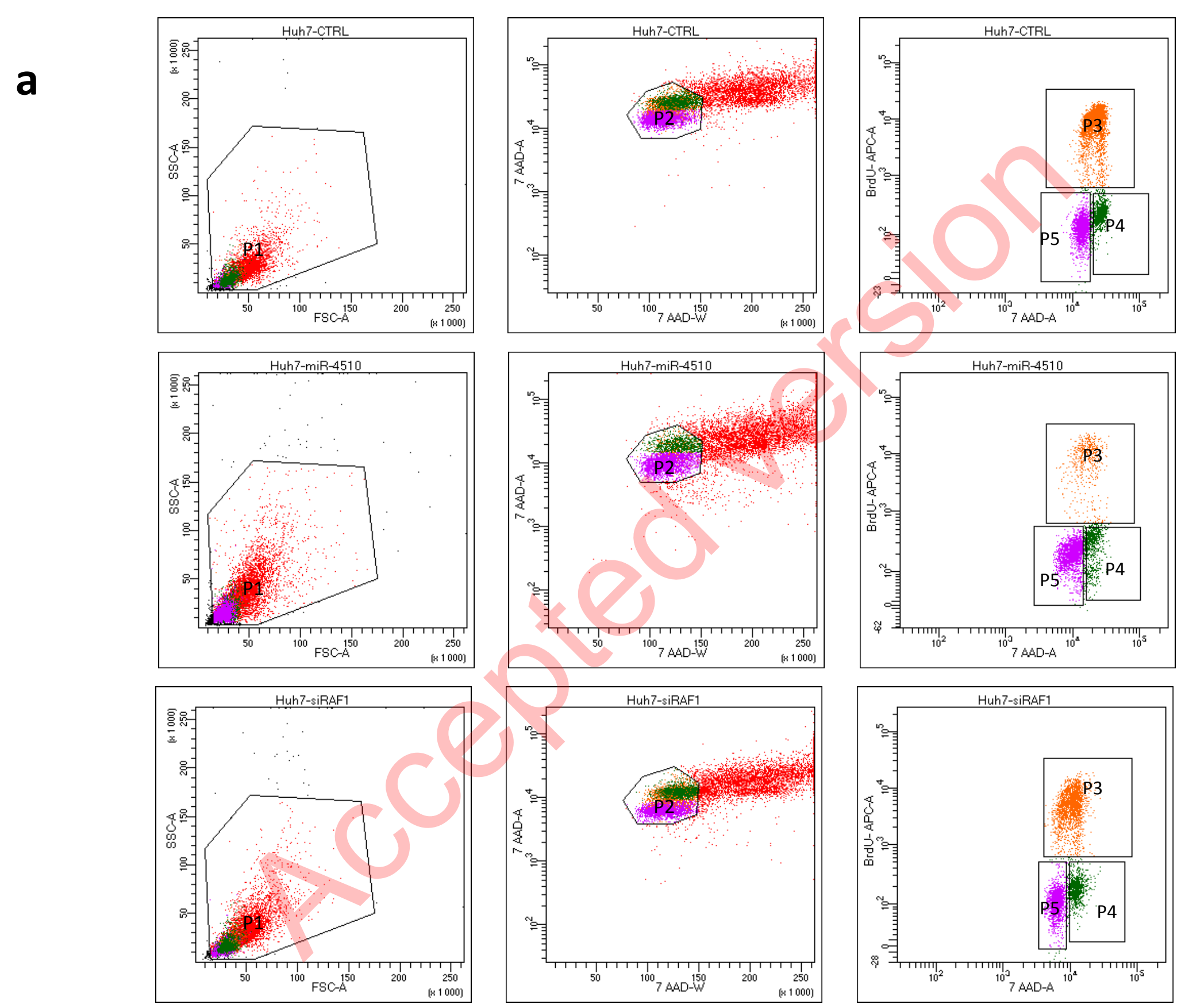

Ghousein A et al, Supplementary Figure S4 


\section{a Huh7 cells}

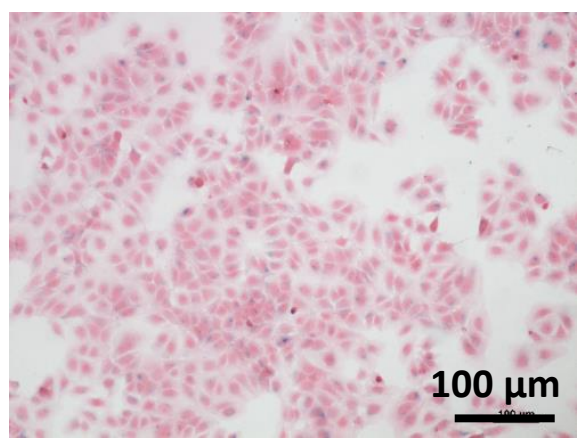

CTRL

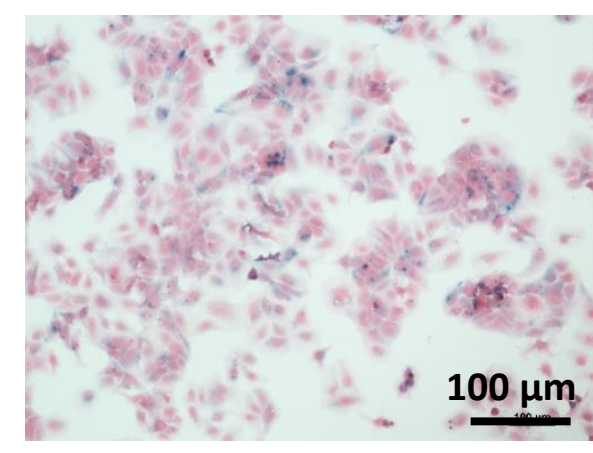

miR-4510

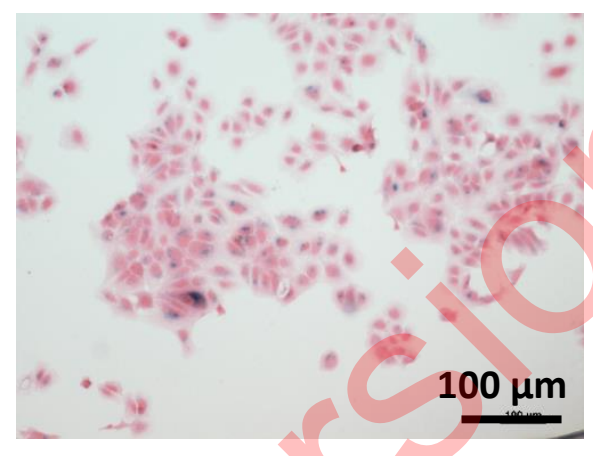

SiRAF1

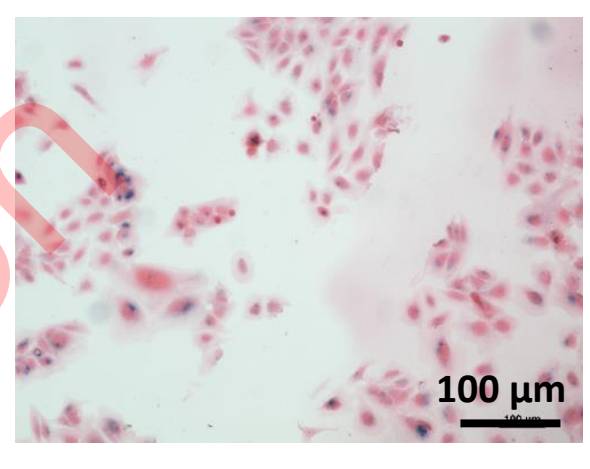

miR-624-5p

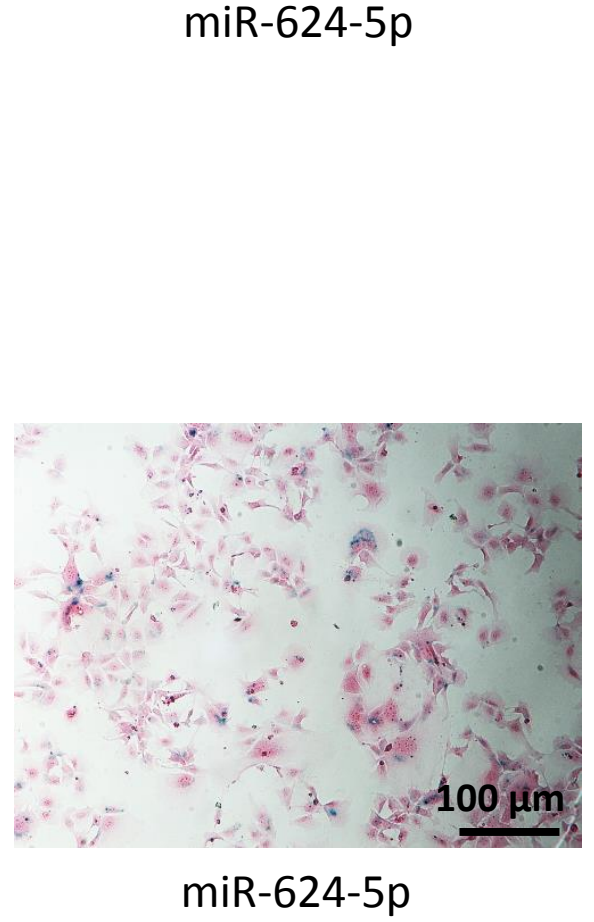

miR-624-5p

b Hep3B cells

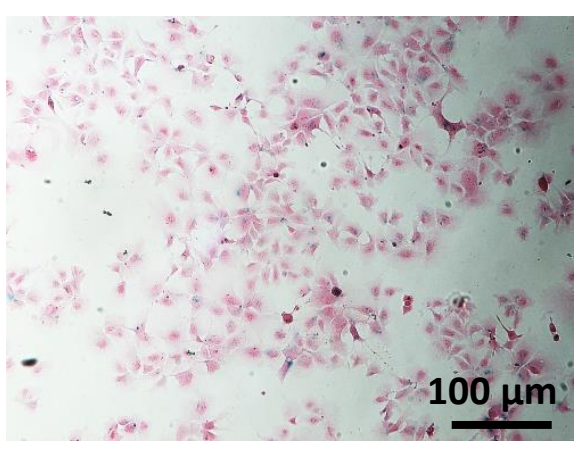

CTRL

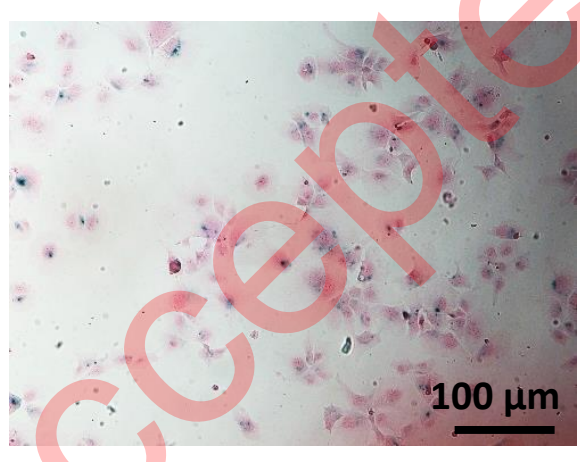

miR-4510

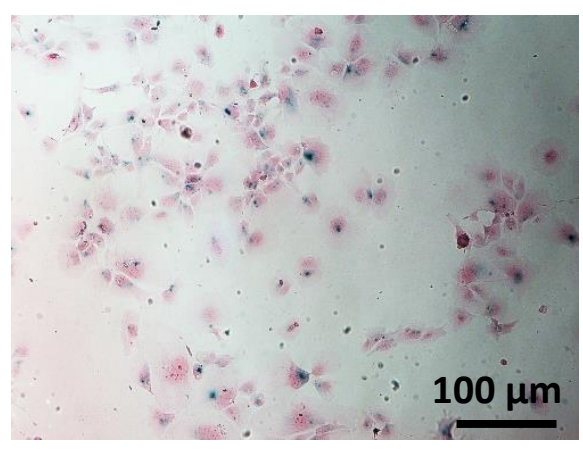

siRAF1

Ghousein A et al, Supplementary Figure S5 
a
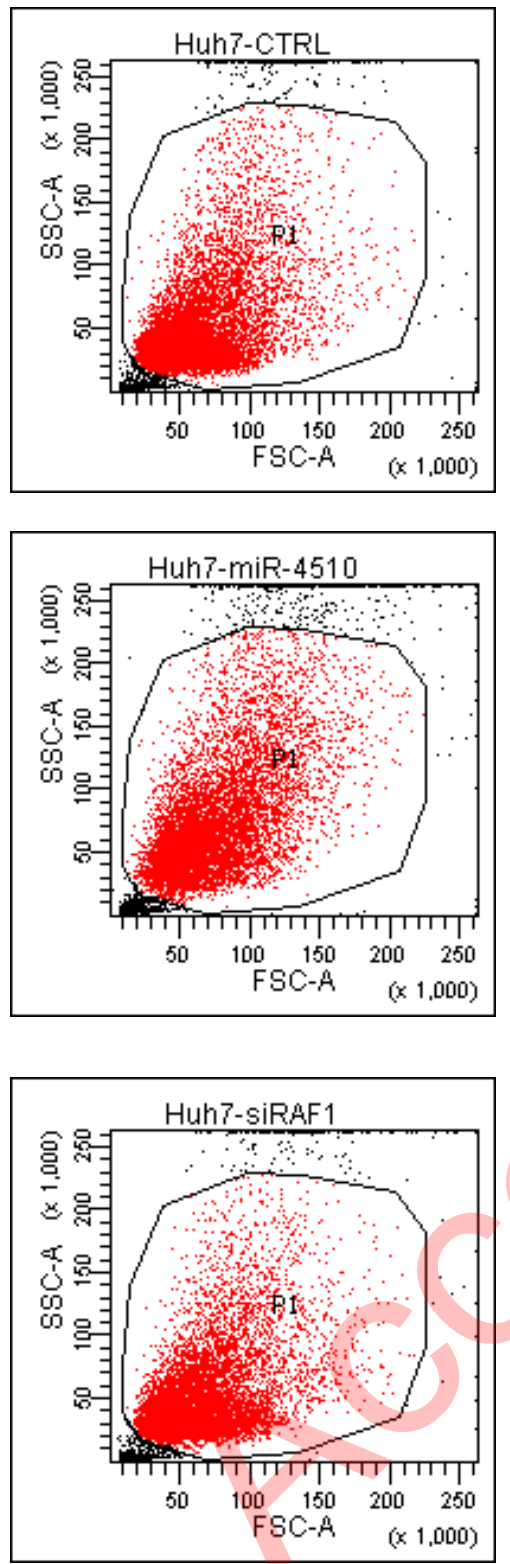
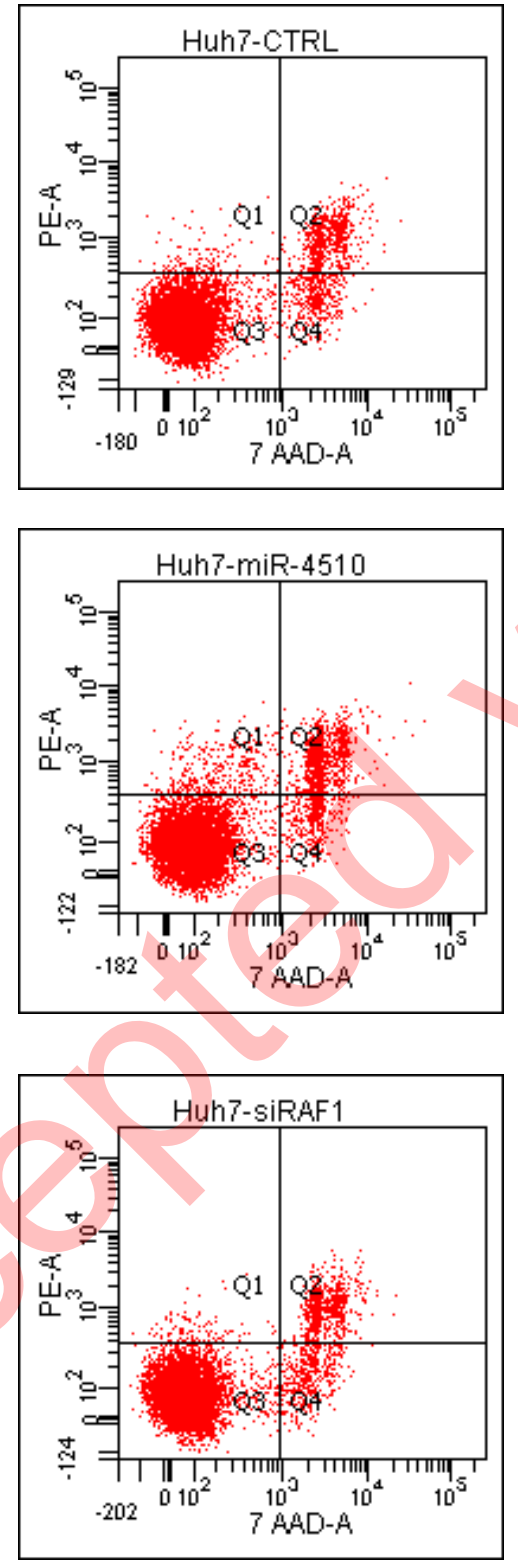

\section{Huh7 cells}
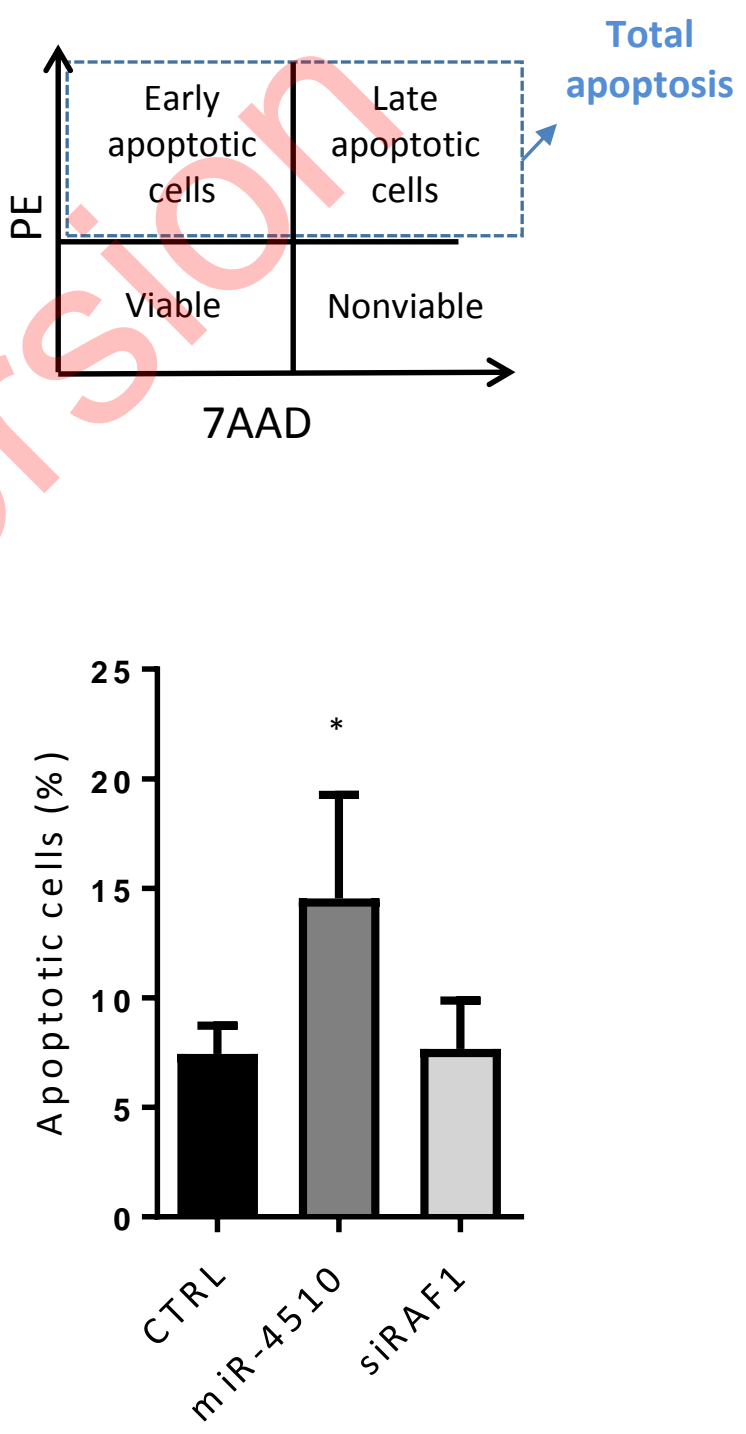
b
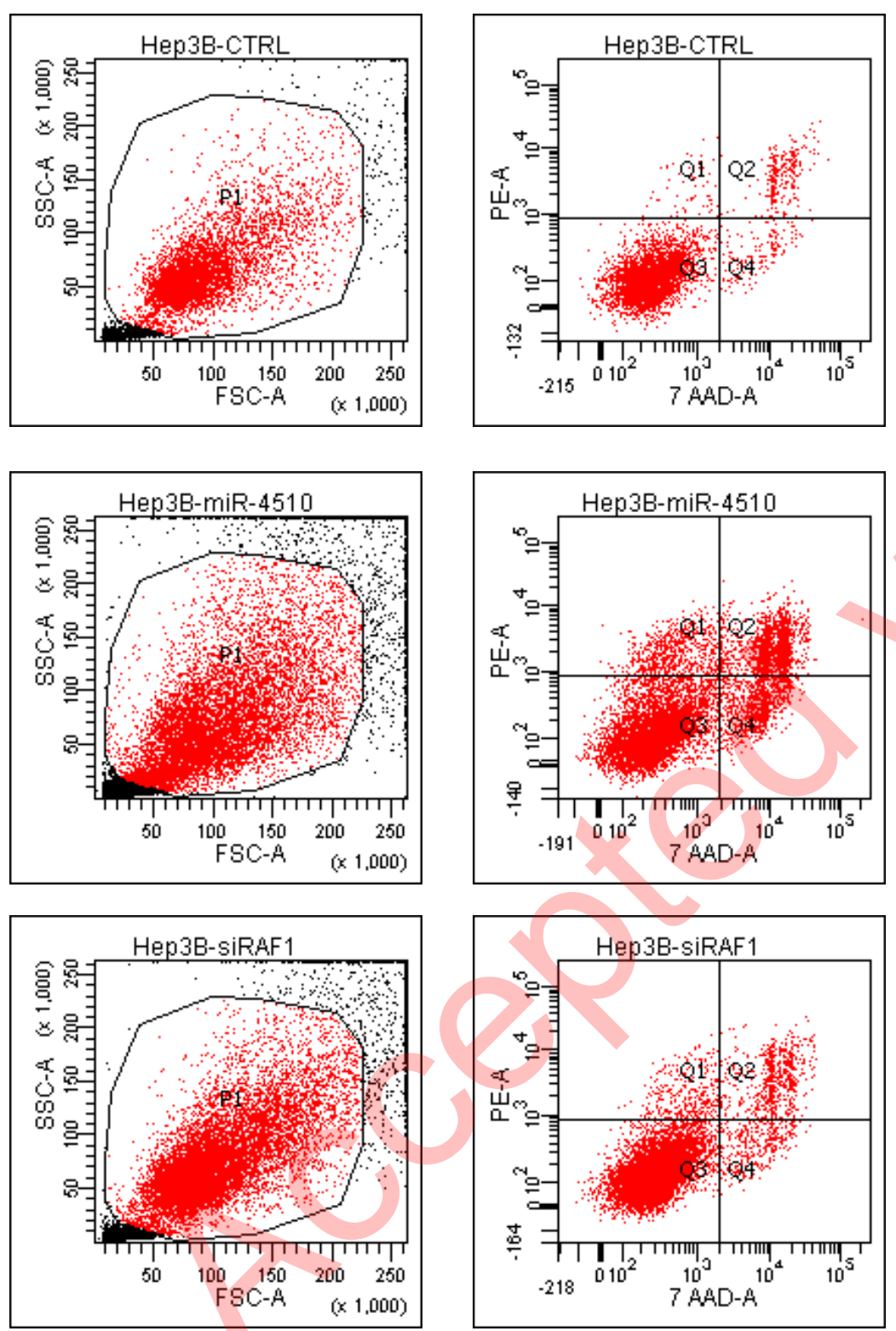

\section{Hep3B cells}
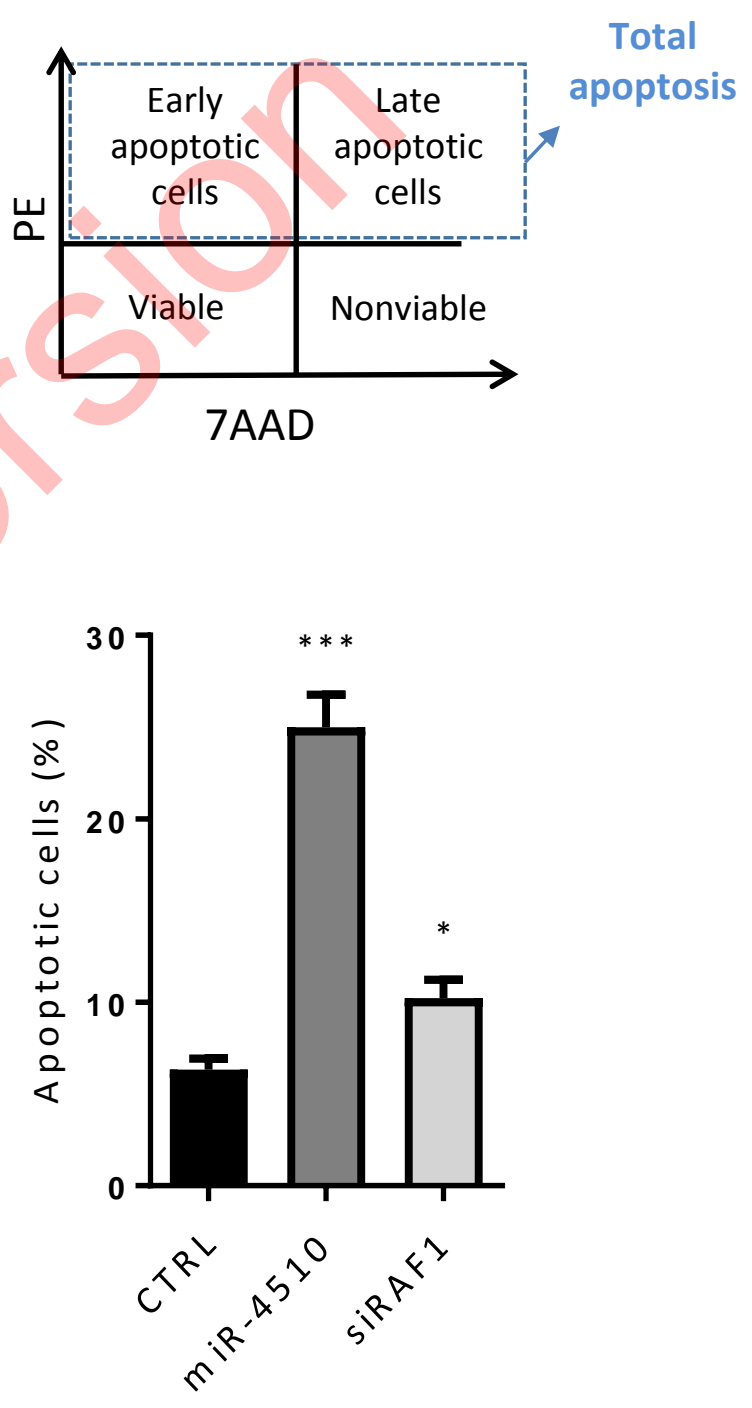
a

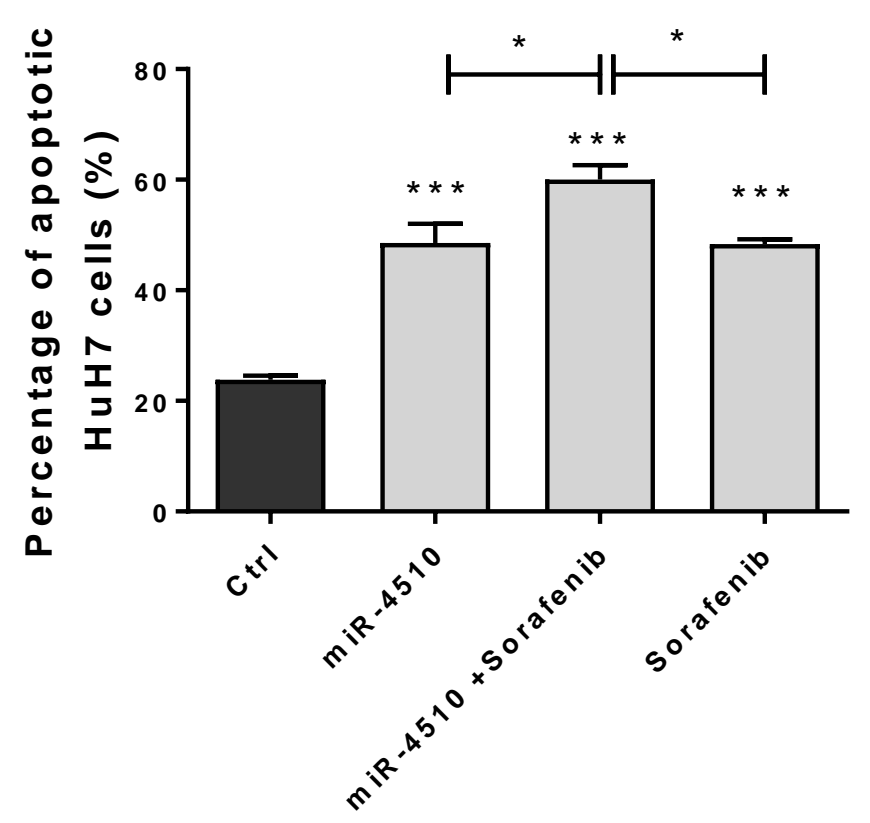

c

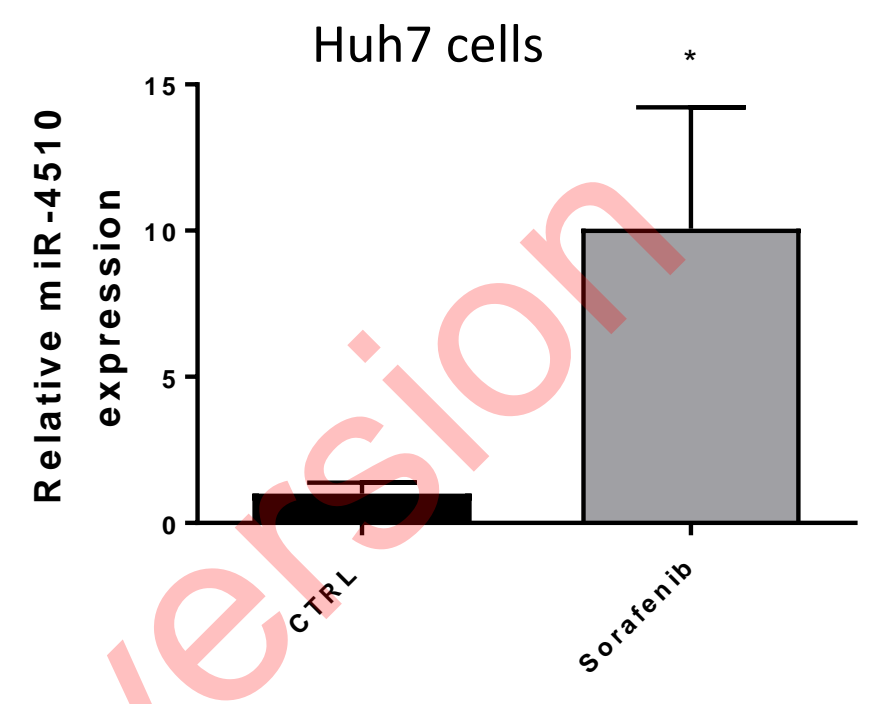

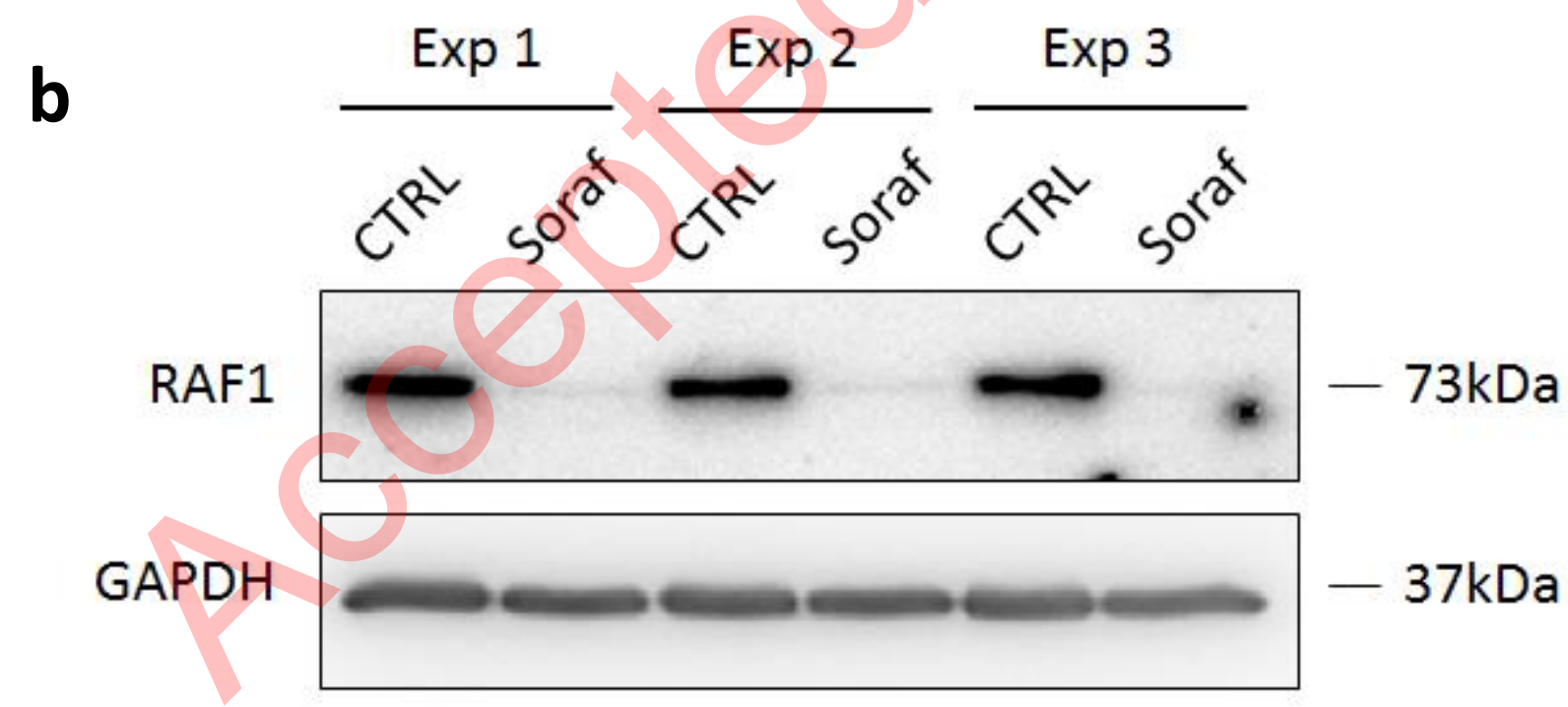

Ghousein A et al, Supplementary Figure S7 
Huh7 cells

$\mathrm{T}=\mathrm{Oh}$
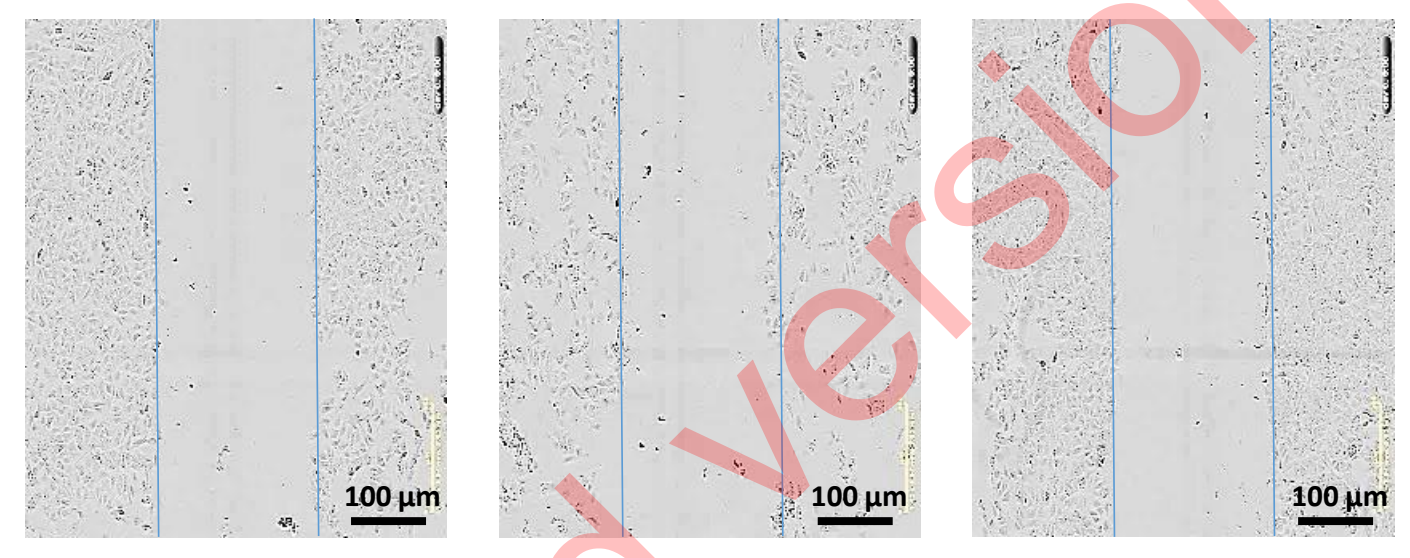

$\mathrm{T}=24 \mathrm{~h}$

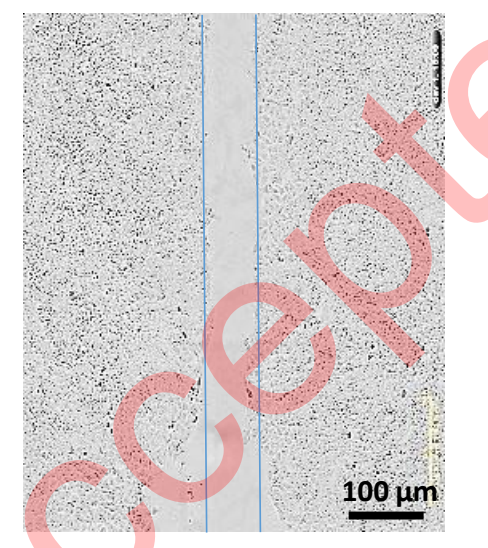

CTRL

miR-4510

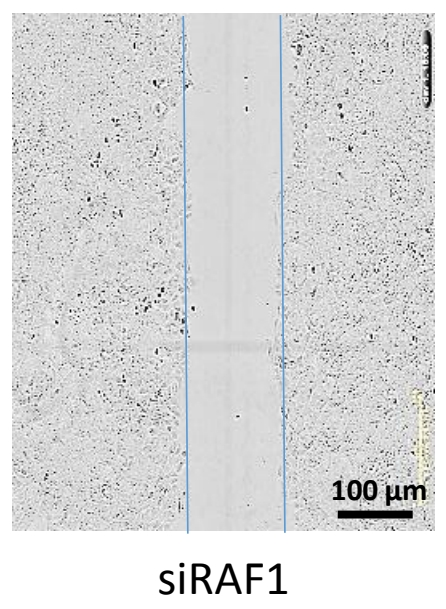

Ghousein A et al, Supplementary Figure $\mathrm{S} 8$ 
b

Huh7 cells

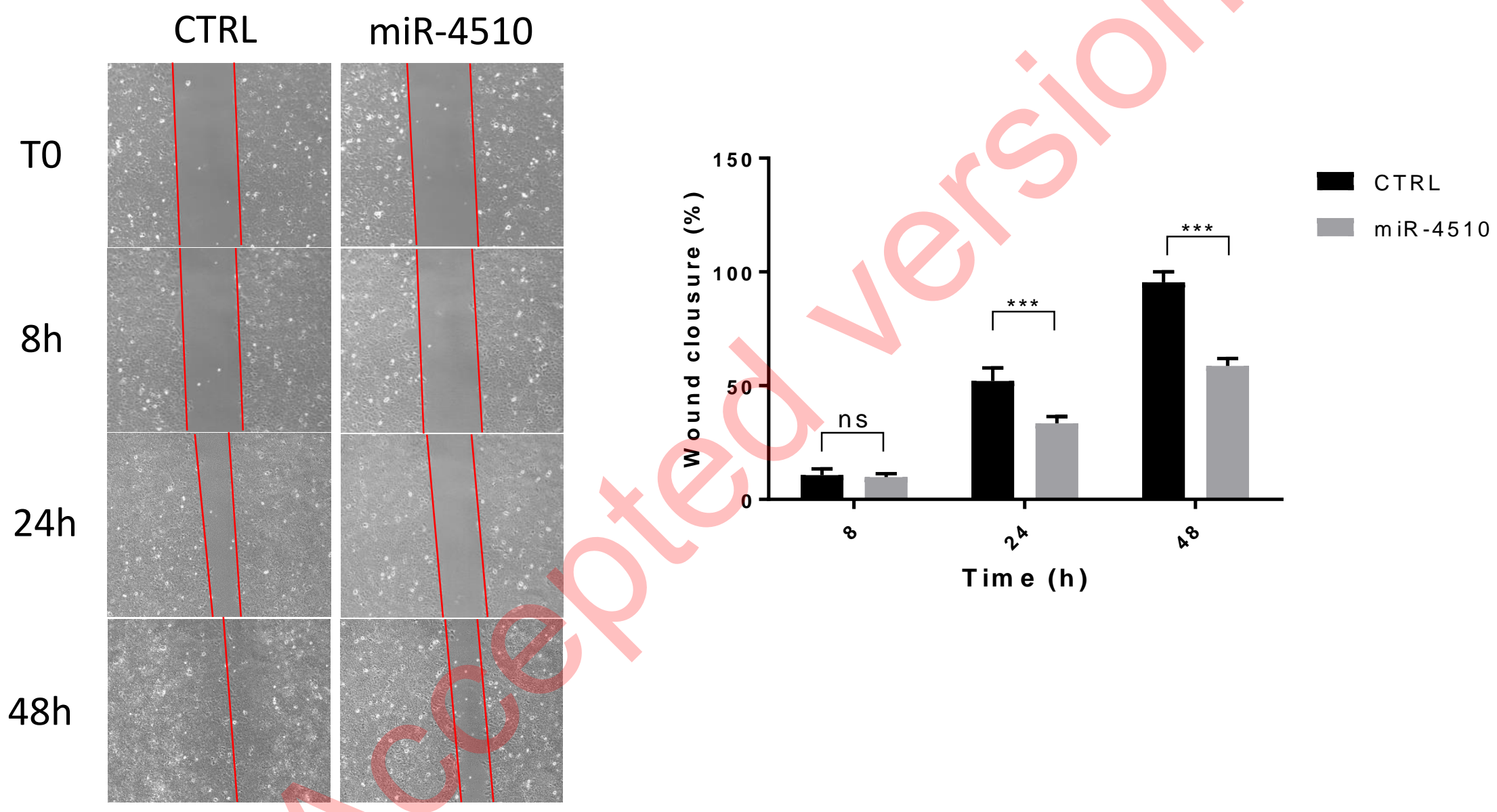


Huh7 cells

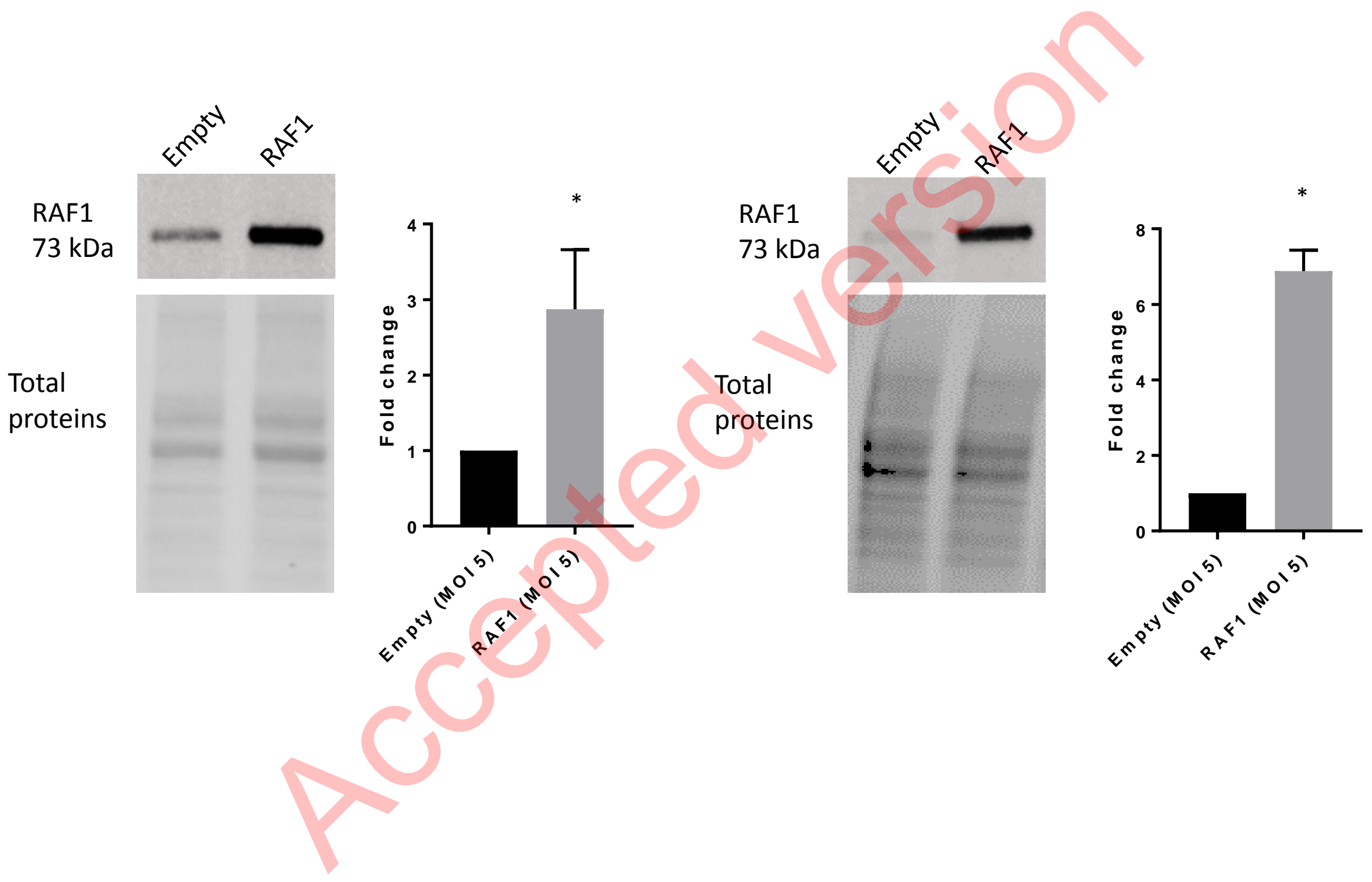

Hep3B cells 
Huh7 cells
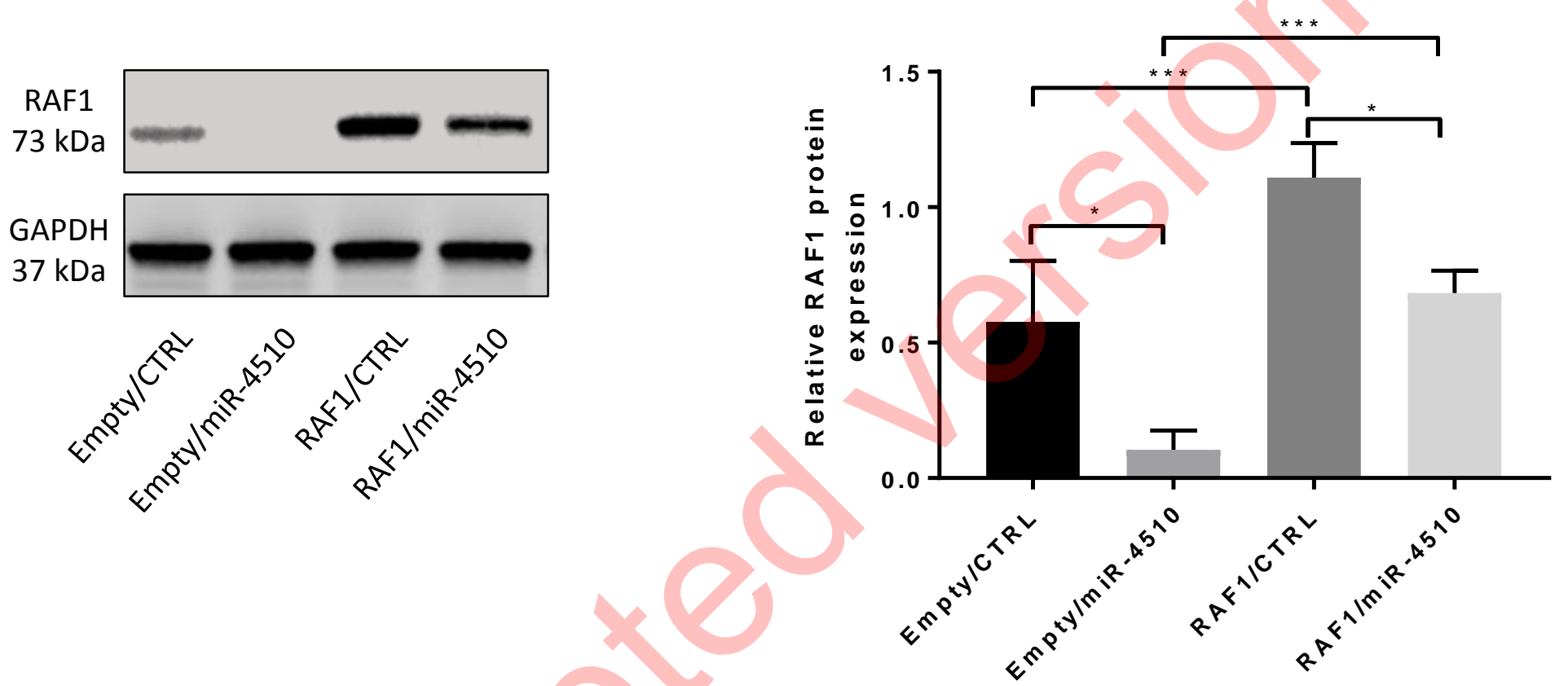

Ghousein A et al, Supplementary Figure S10 
a

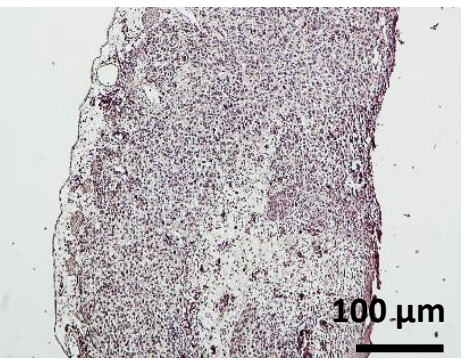

1

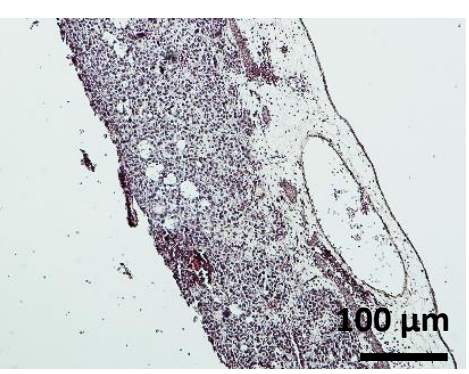

6

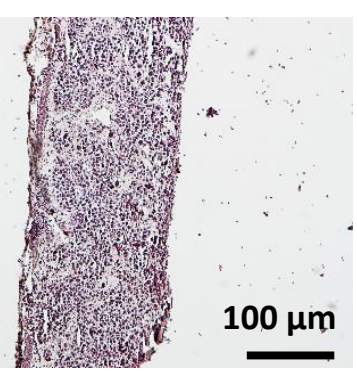

11

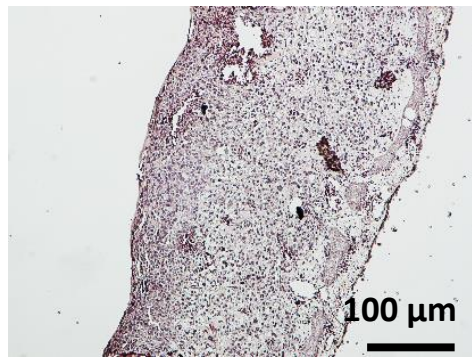

2

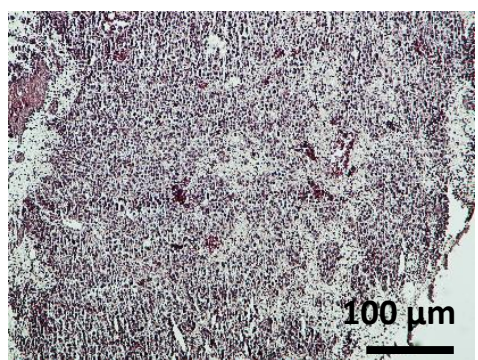

7

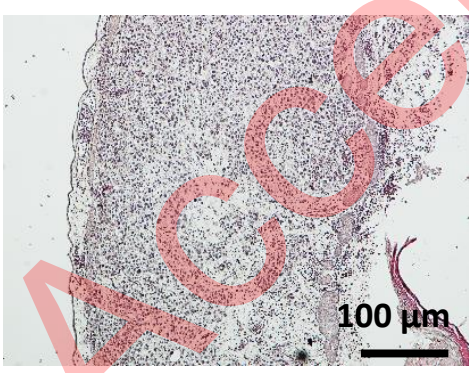

12

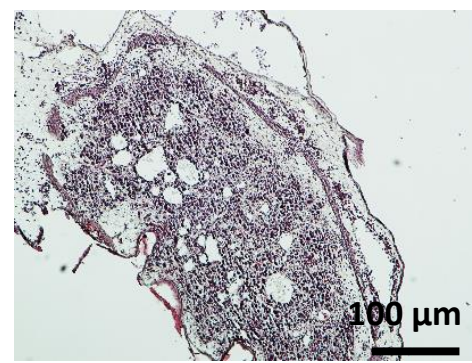

3

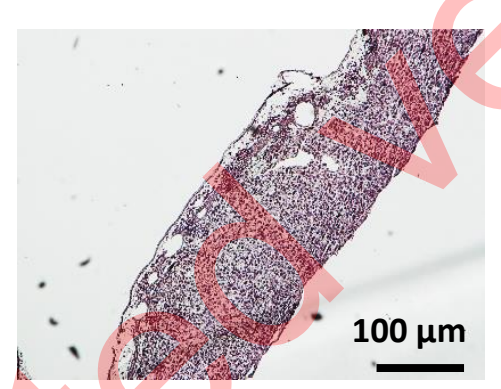

8

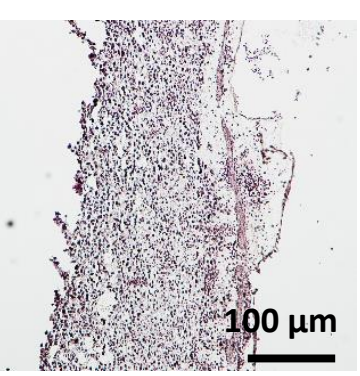

13
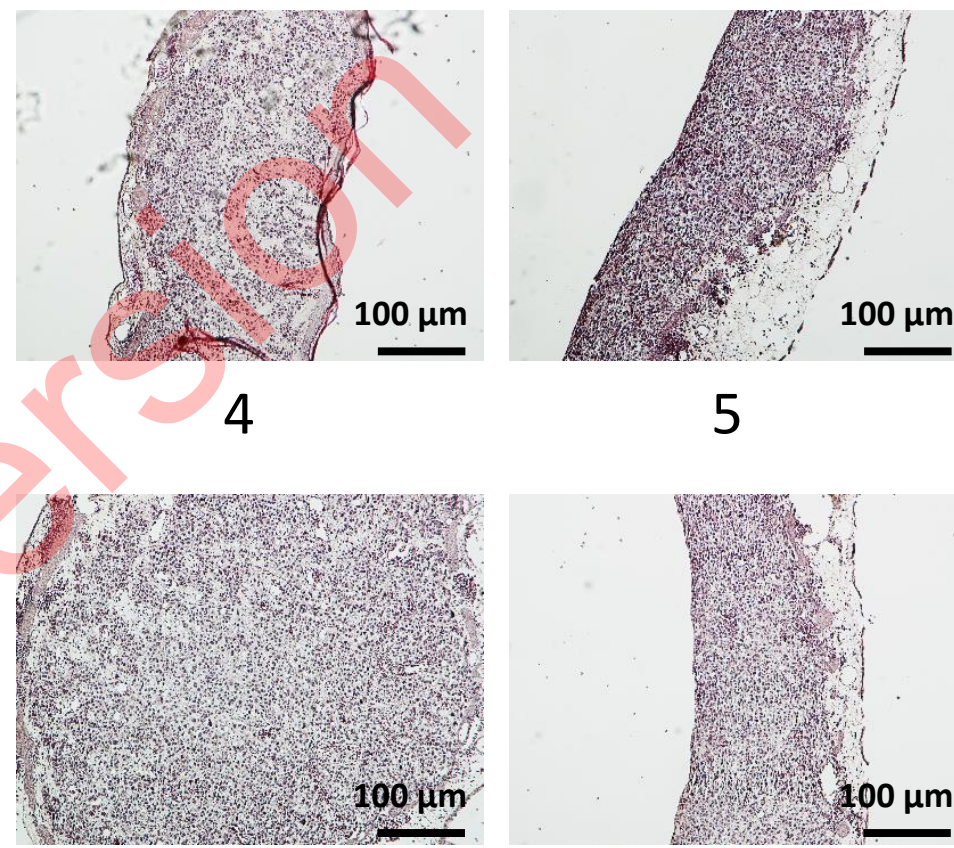

9

10

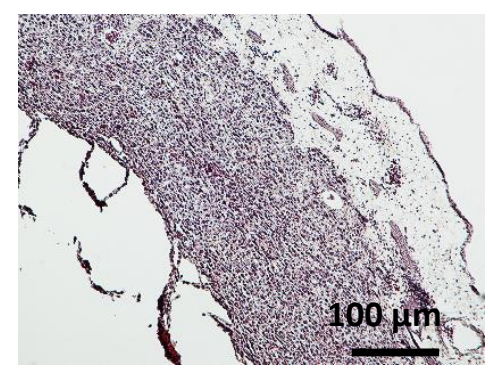

14

Ghousein A et al, Supplementary Figure S11 
b

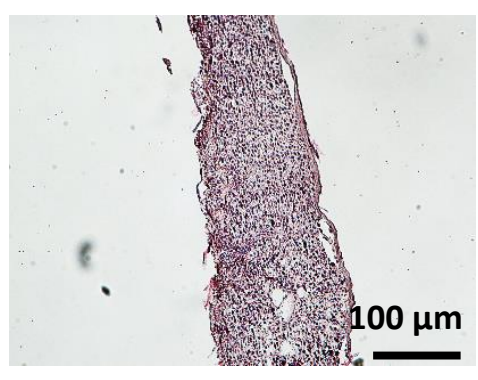

1

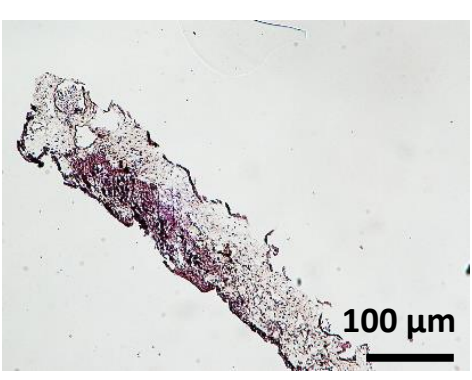

6

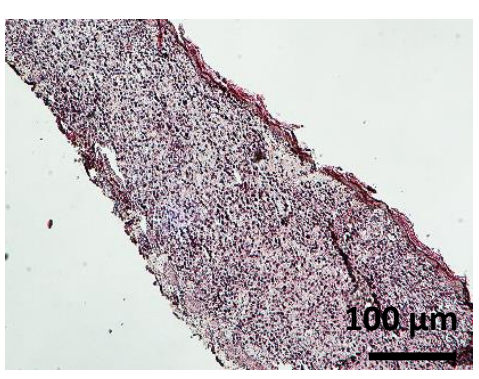

11

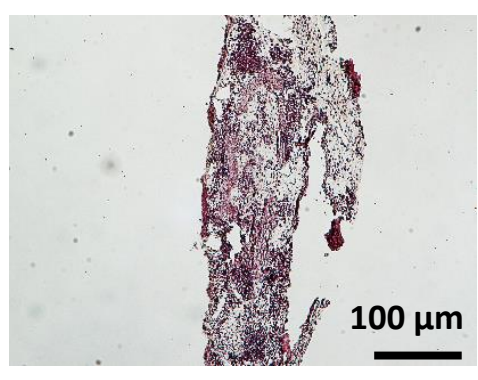

2

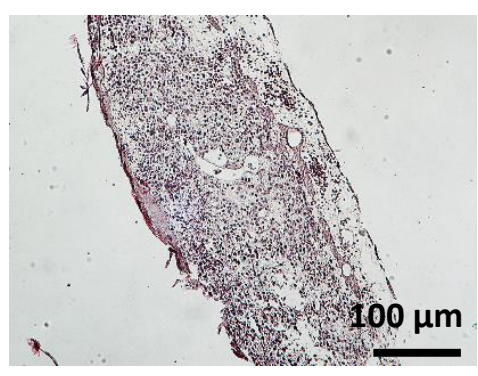

7

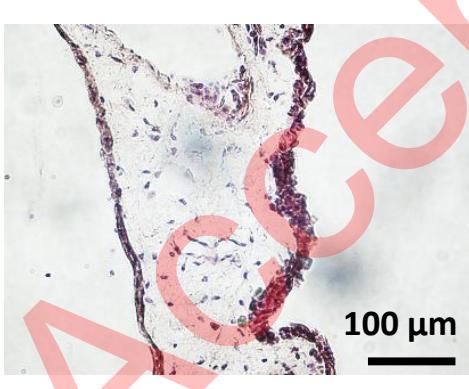

12

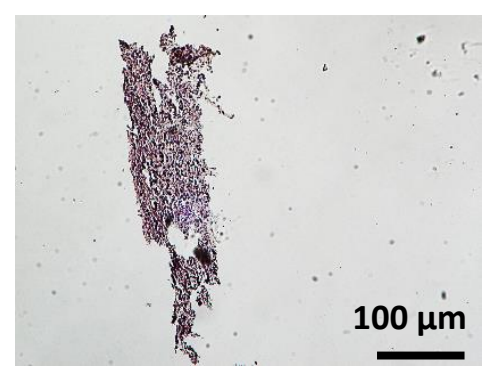

3

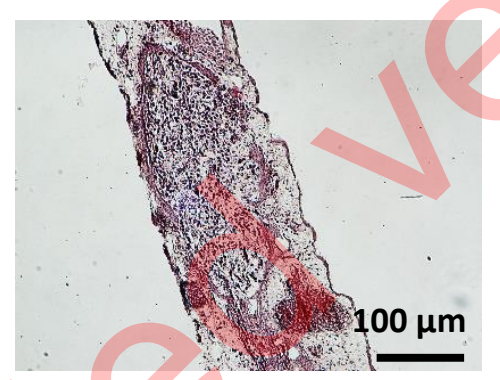

8

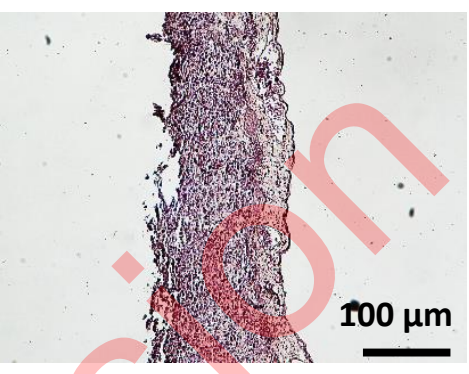

4

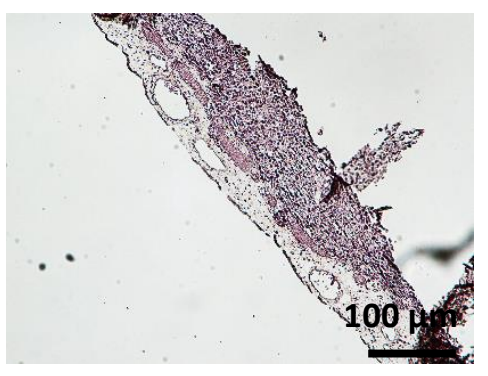

5

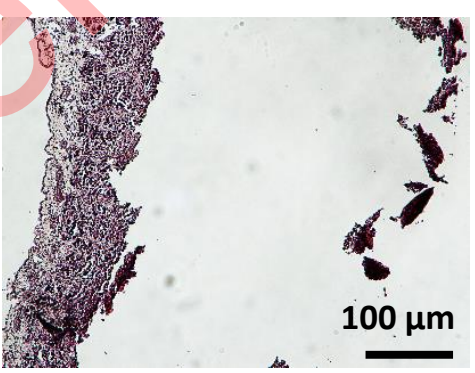

9

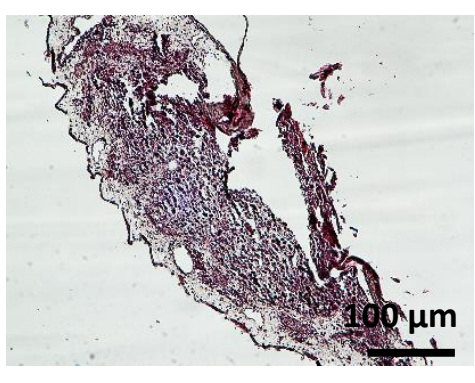

10

Ghousein A et al, Supplementary Figure S11 


\section{C}

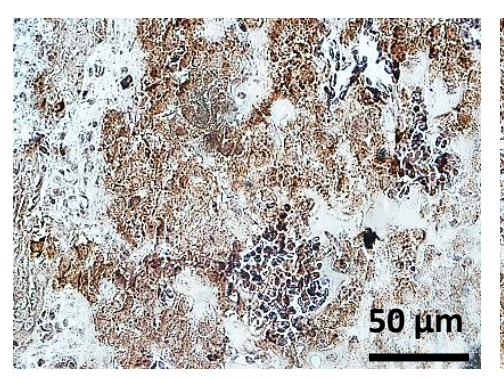

1

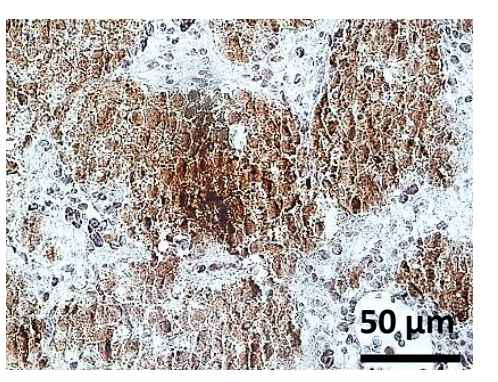

2

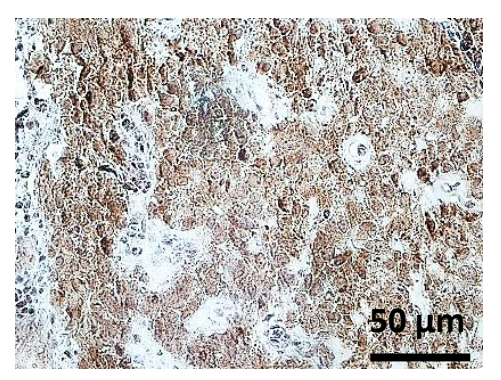

3

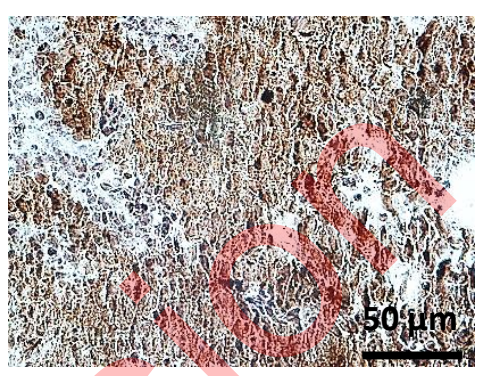

4

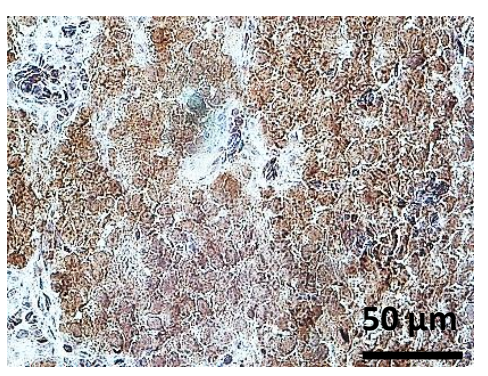

5

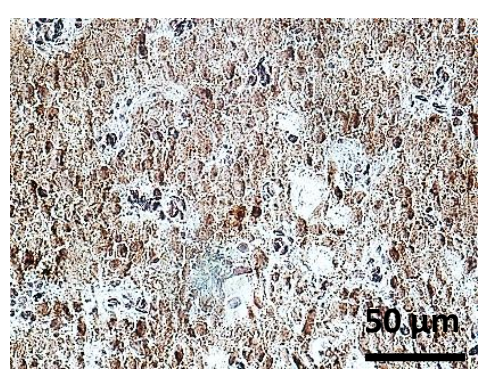

6

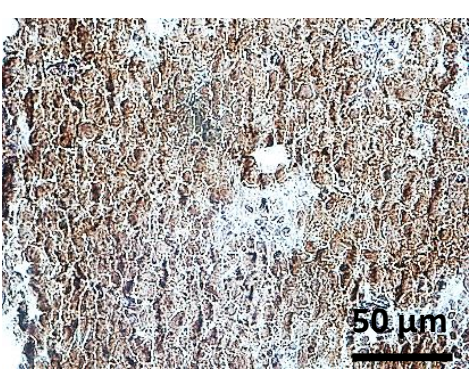

7

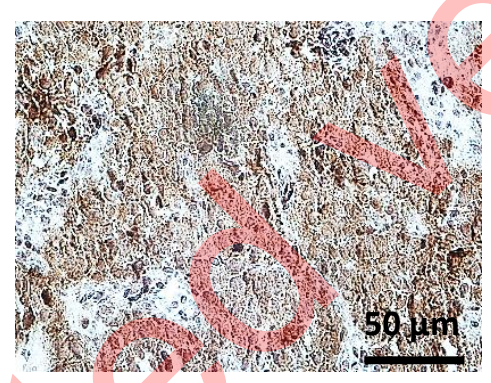

8

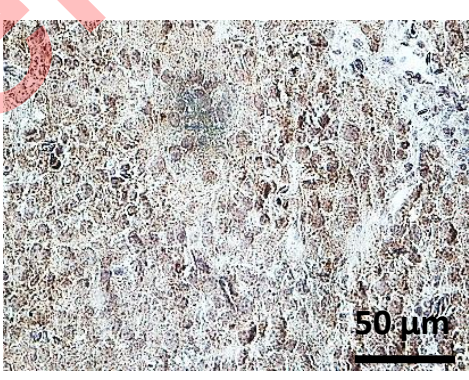

9

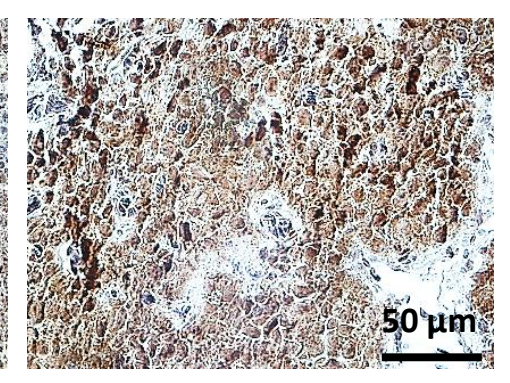

10

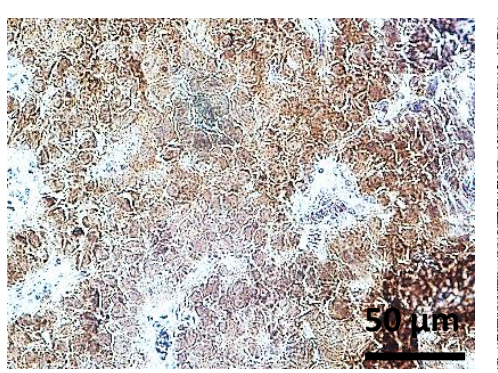

11

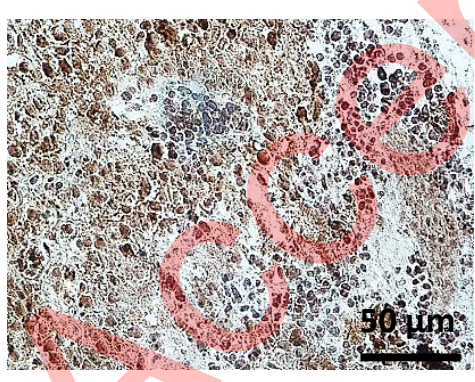

12

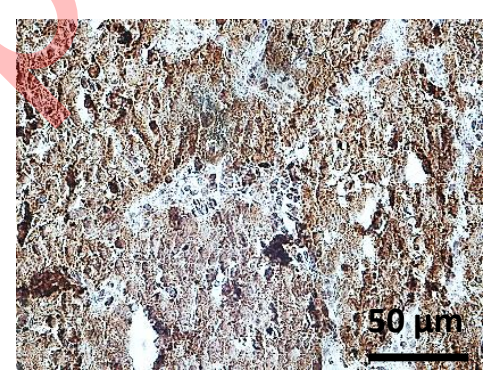

13

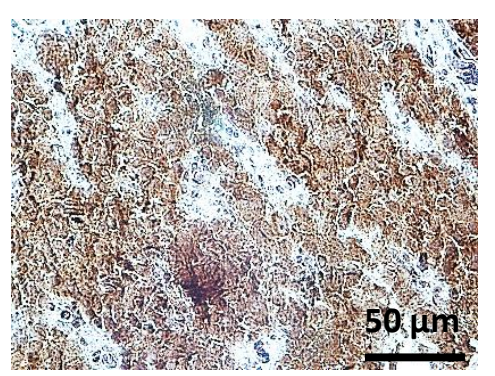

14

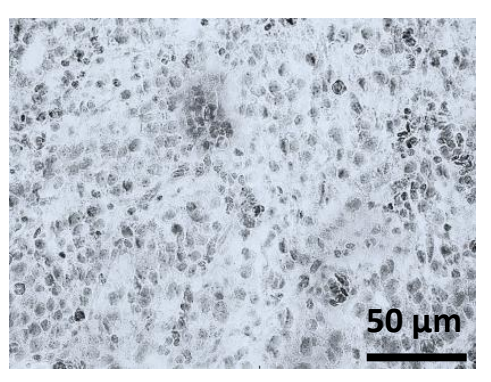

Blank 


\section{d}

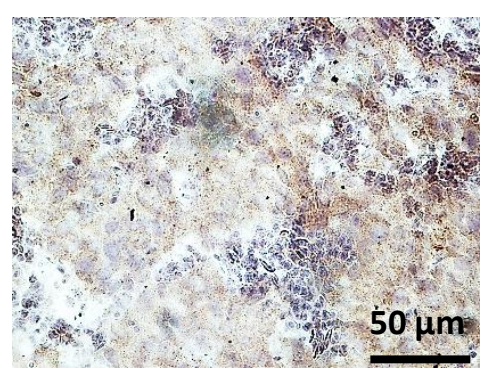

1

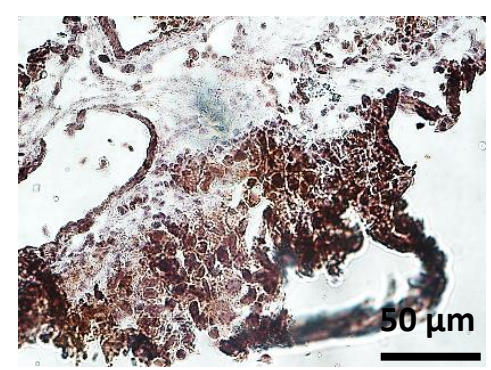

6

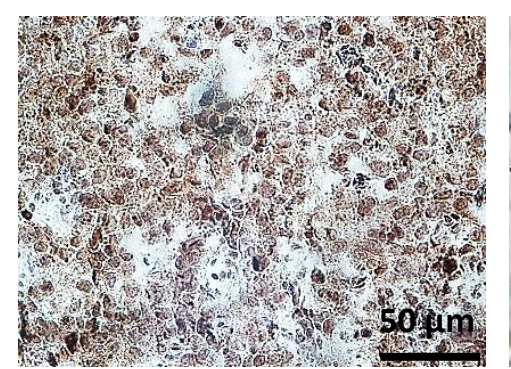

11

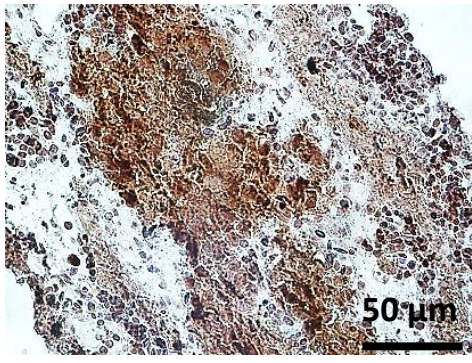

2

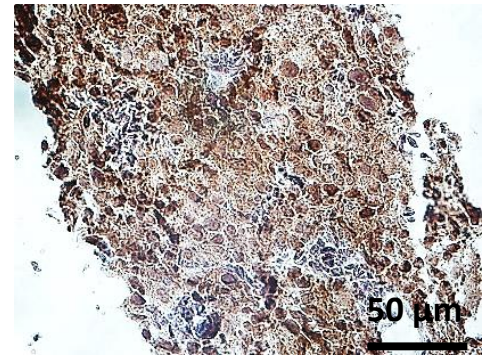

3

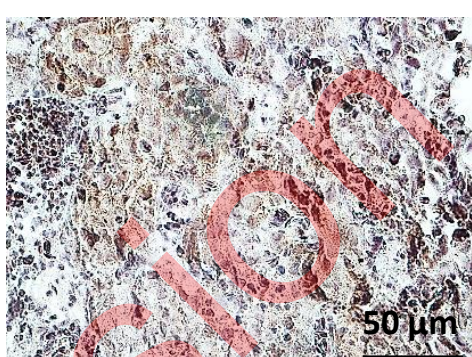

4

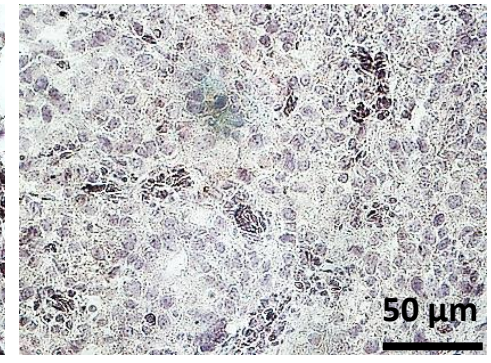

5

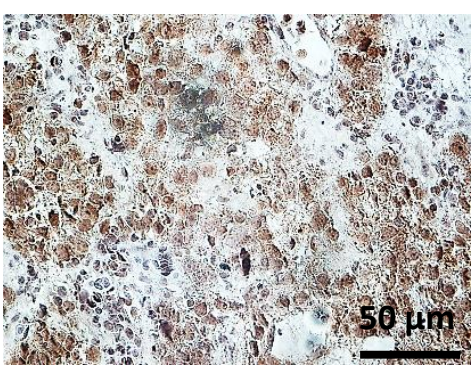

7

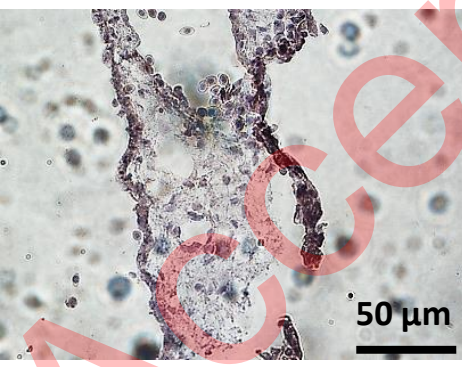

12

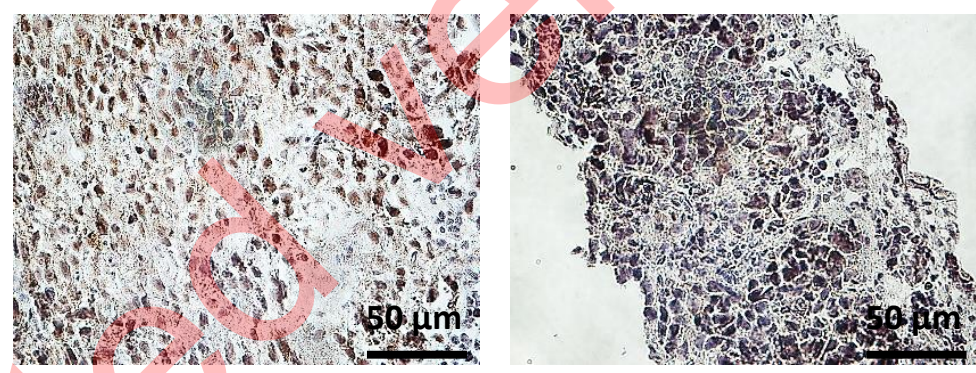

9

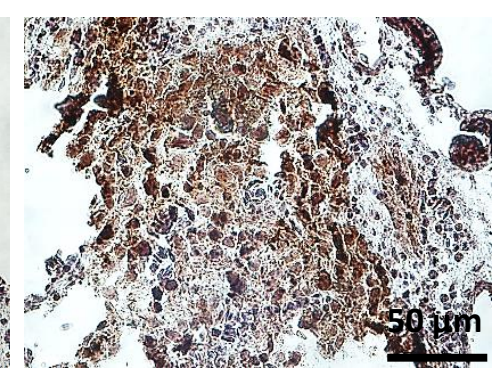

10

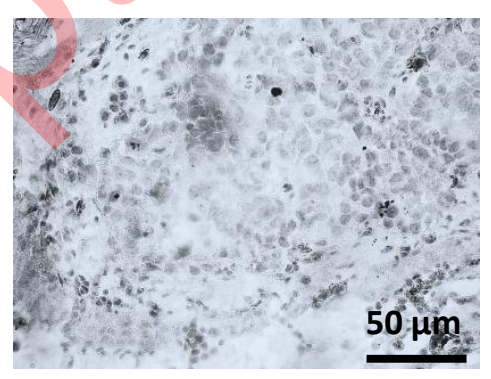

Blank 


\section{e}

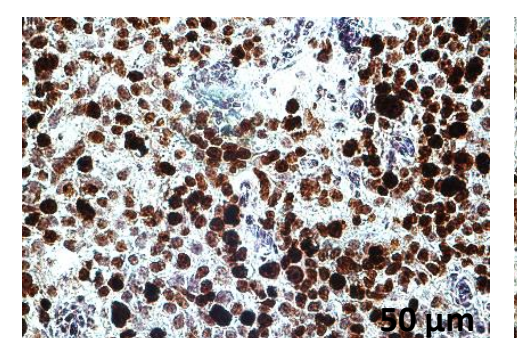

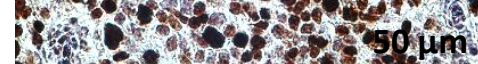

1

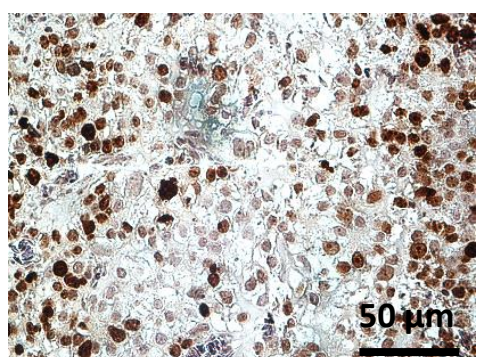

2

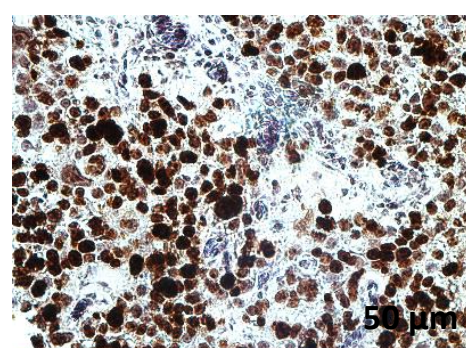

3

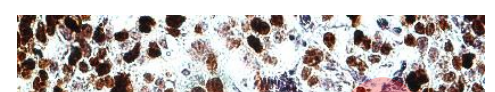
Ax $=$ -

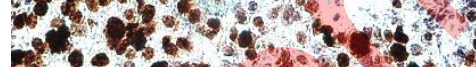

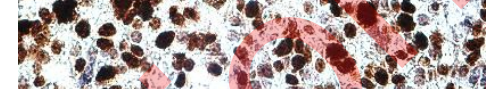
If? $27 \%$

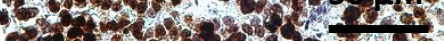

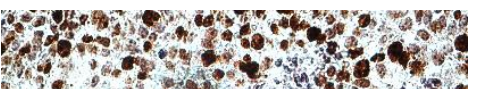

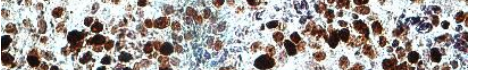

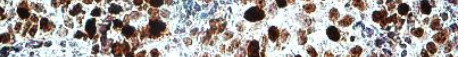

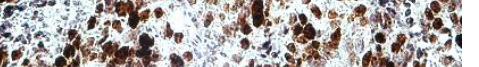
Dw

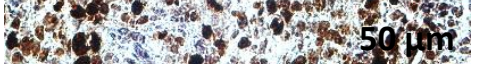
2. 3. 5

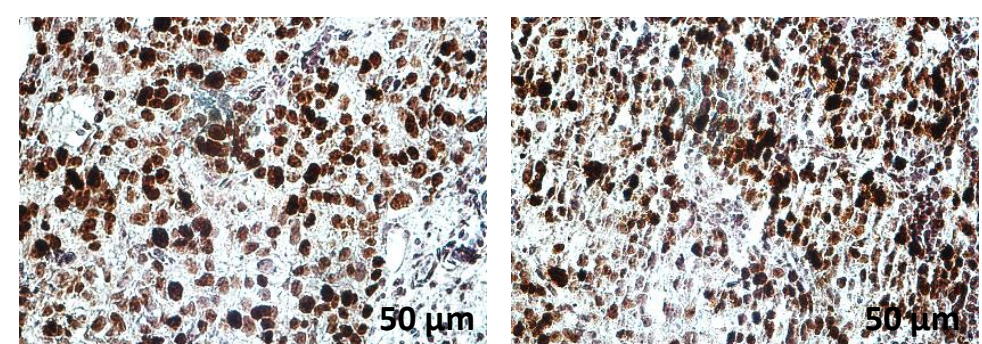

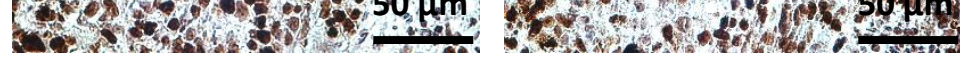

6
7

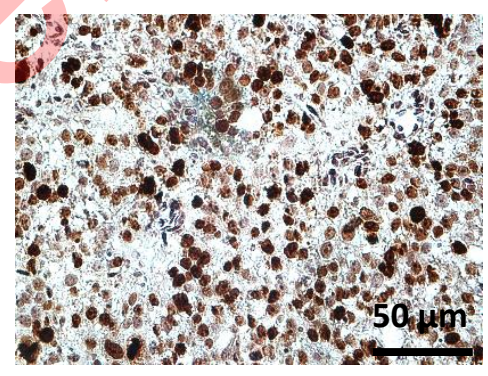

9

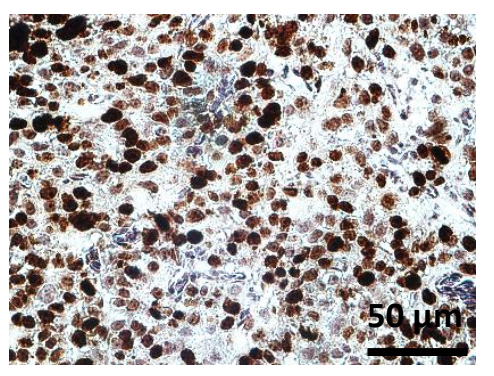

10
Holnos

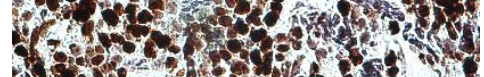

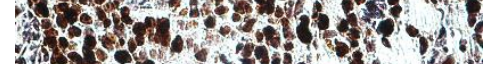

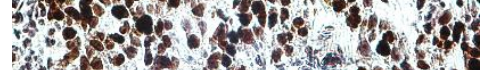

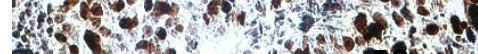
- 27 ind

11

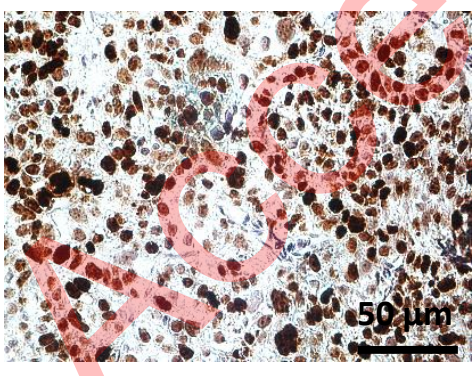

12

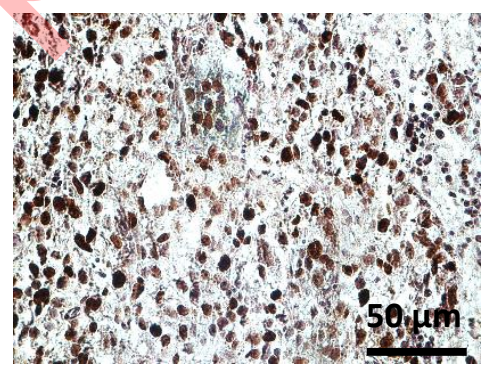

13

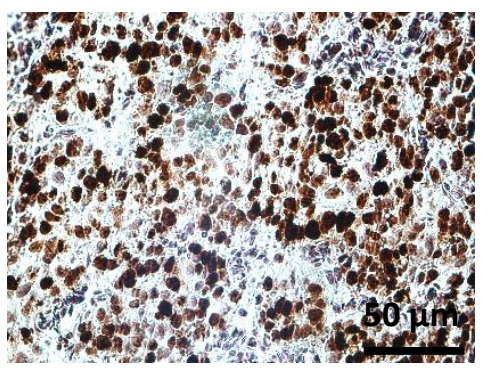

14

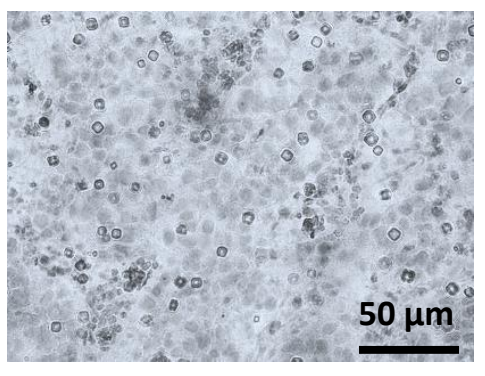

Blank

Ghousein A et al, Supplementary Figure S11 


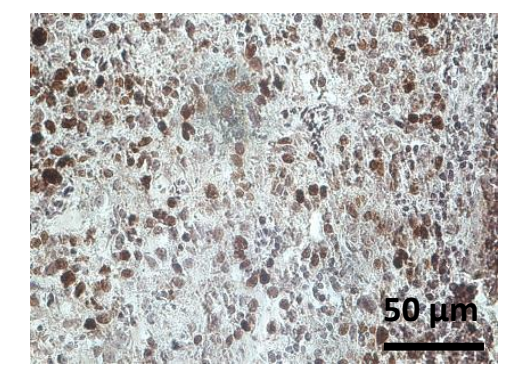

1

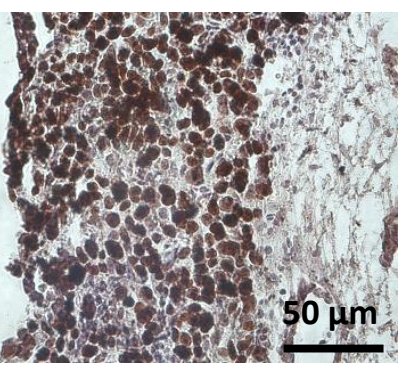

6

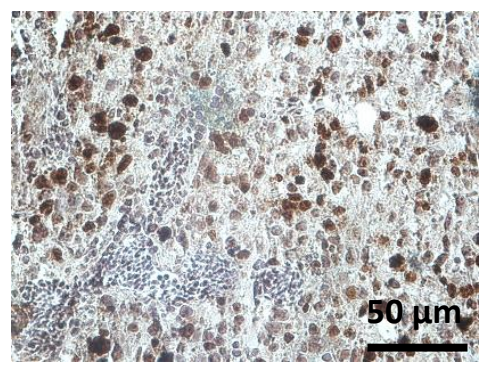

11

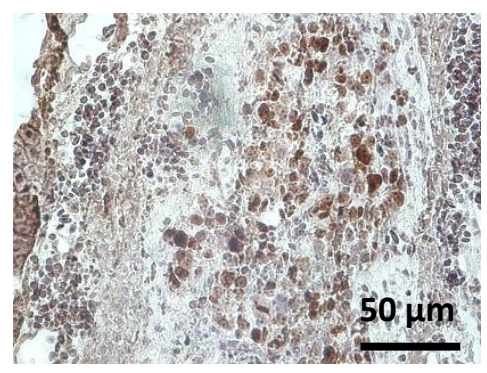

2

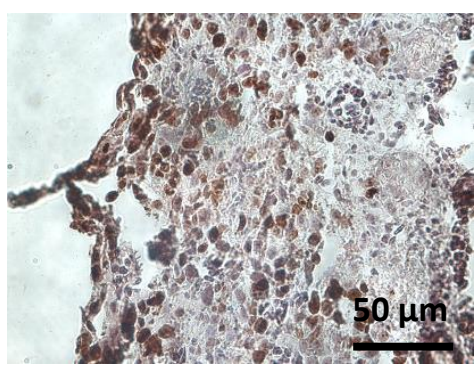

3

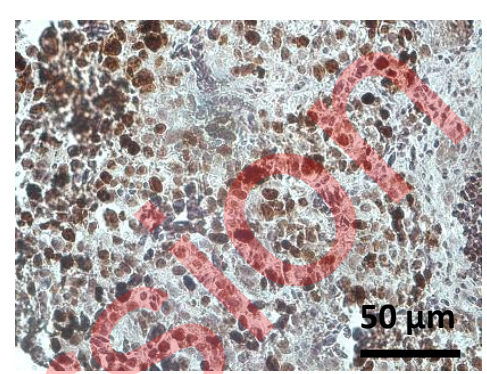

4

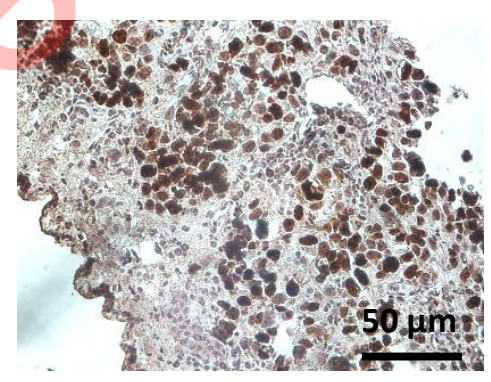

9

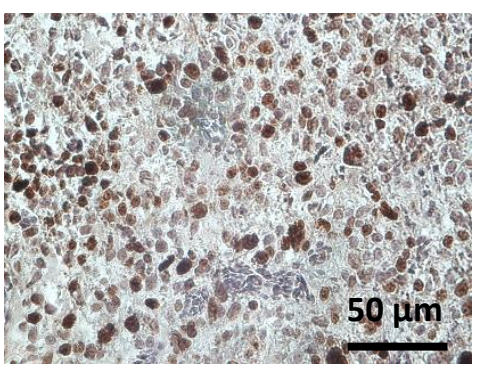

5

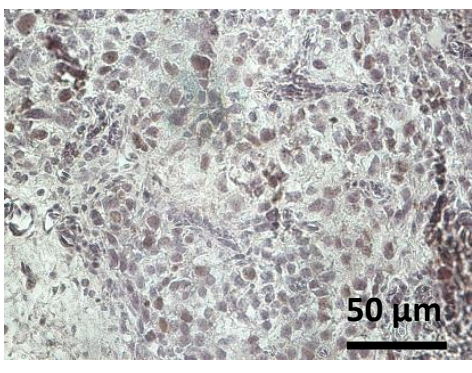

10

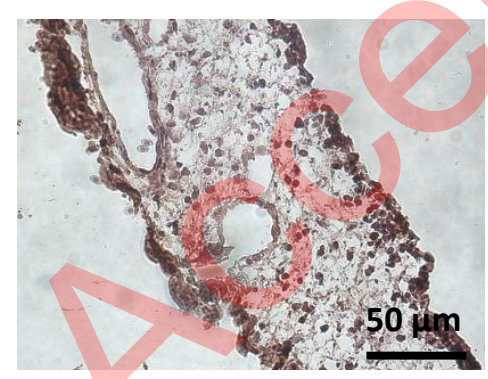

12

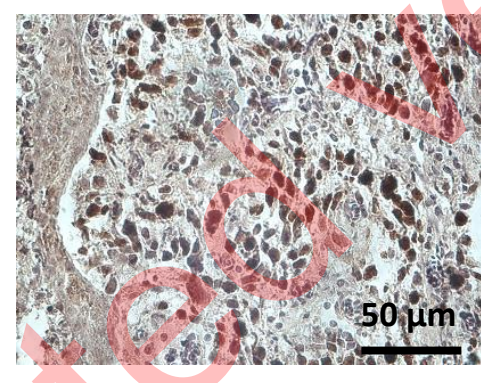

8

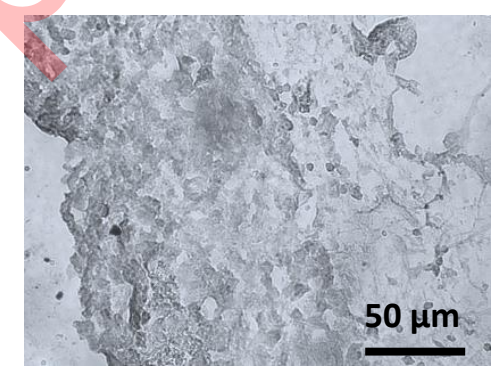

Blank

Ghousein A et al, Supplementary Figure S11 


\section{a}

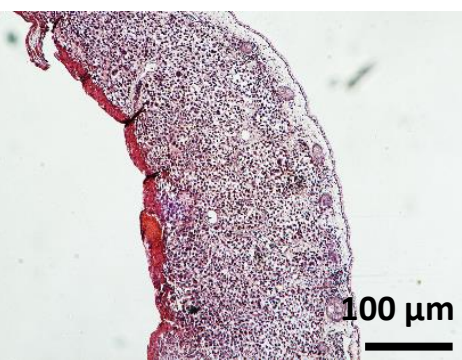

1

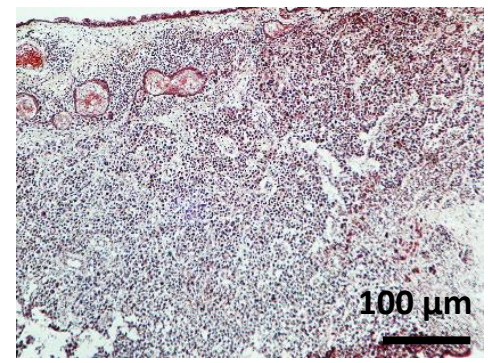

b

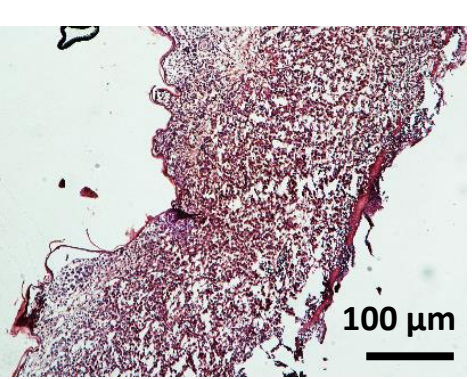

1

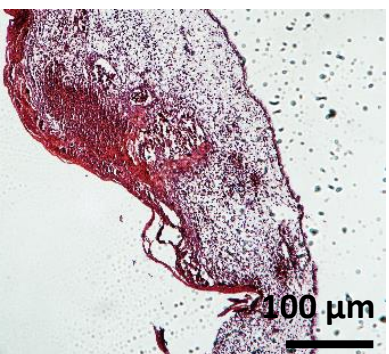

6

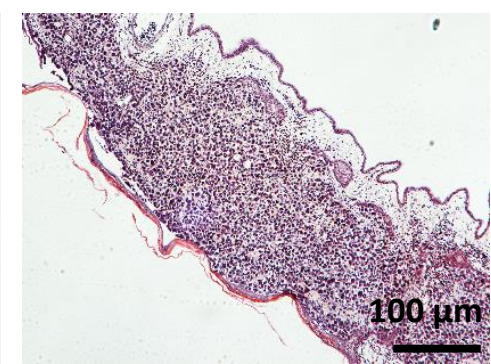

2

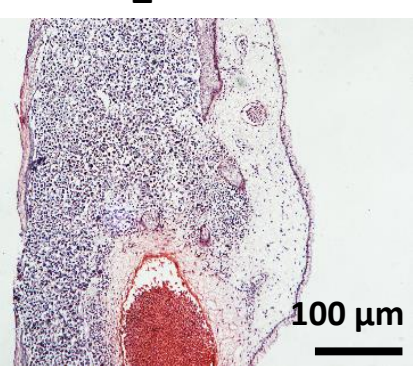

7

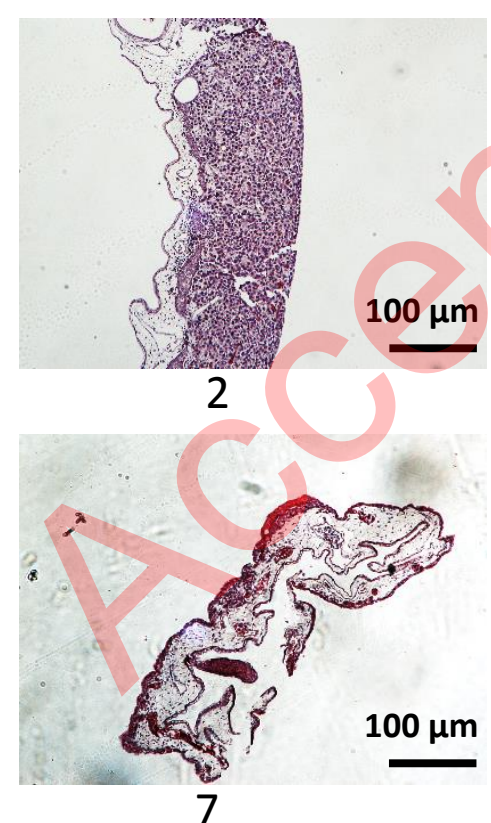

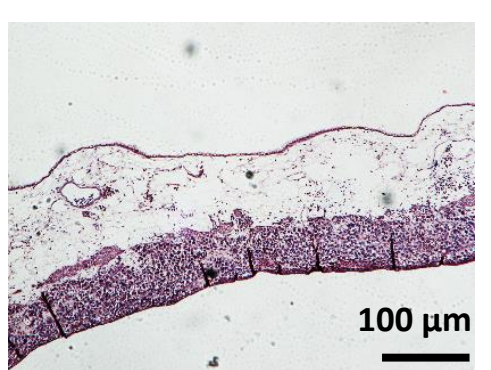

3

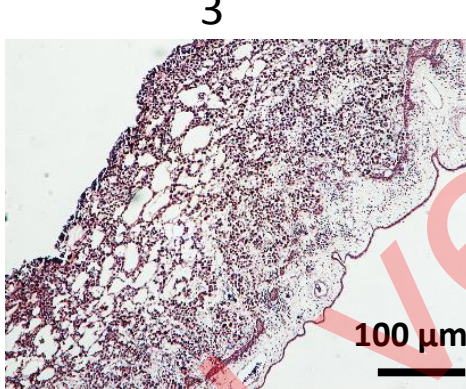

8

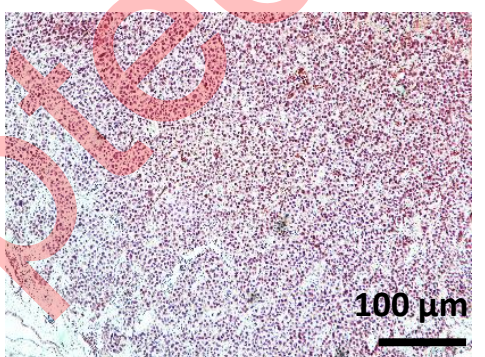

3

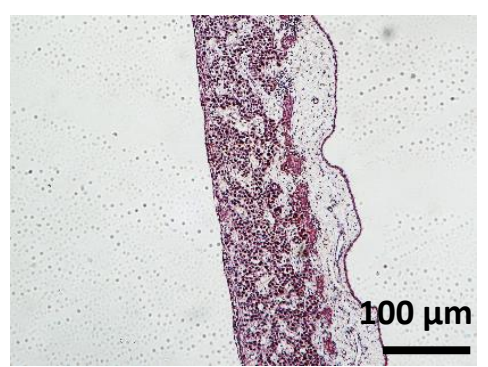

8

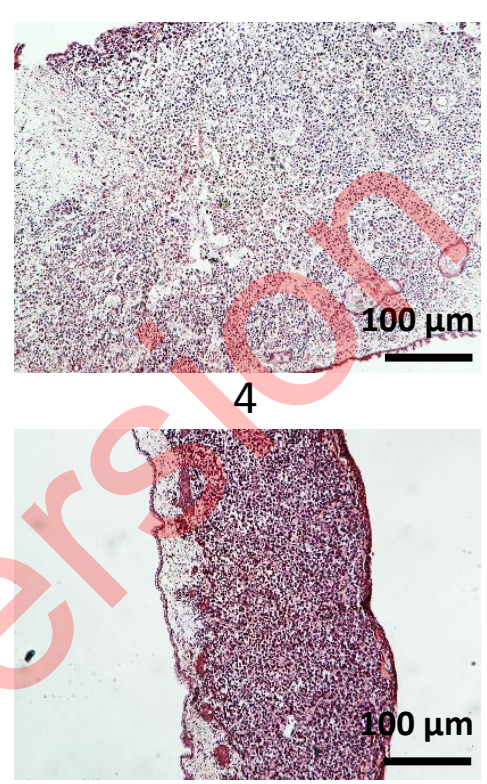

9
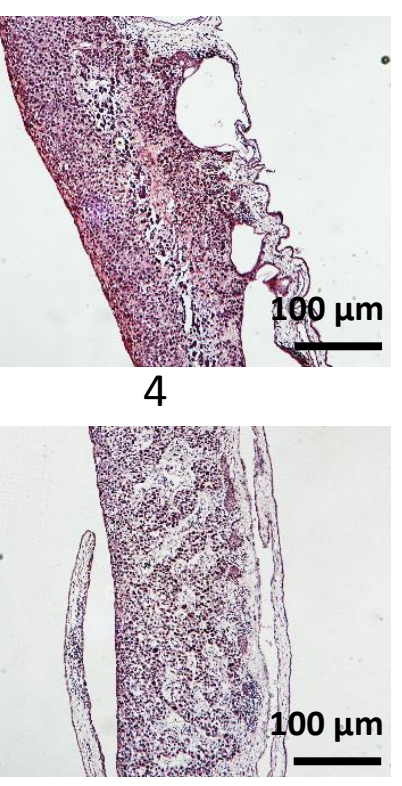

9

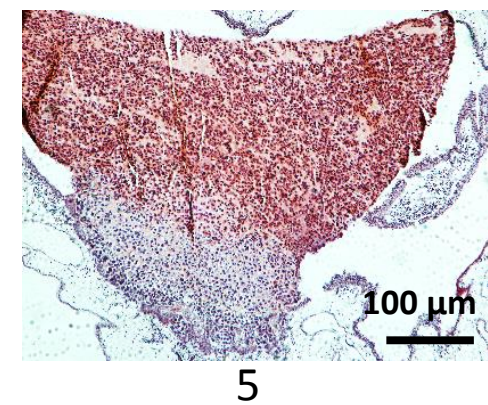

Ghousein A et al, Supplementary Figure S12 


\section{C}

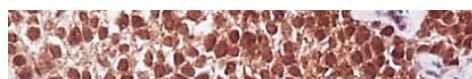
$20.010 \mathrm{ng}$

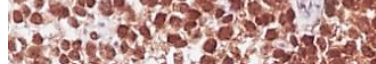

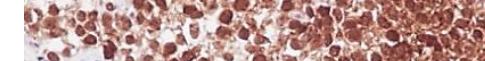

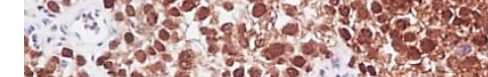

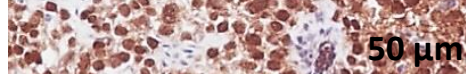

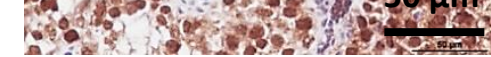
1

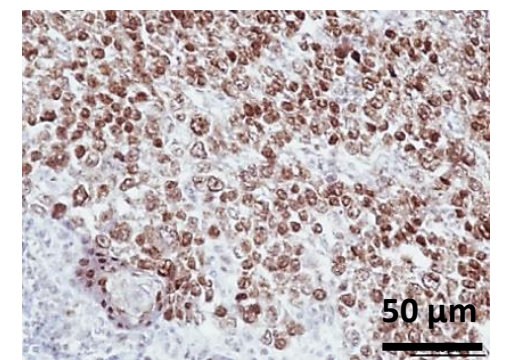

6

1

\section{8} o. gresonsos.

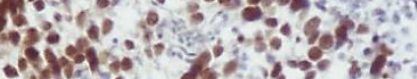
- P o s th

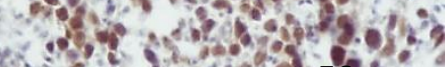

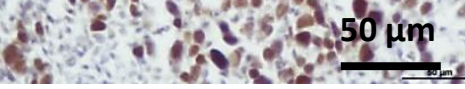
6

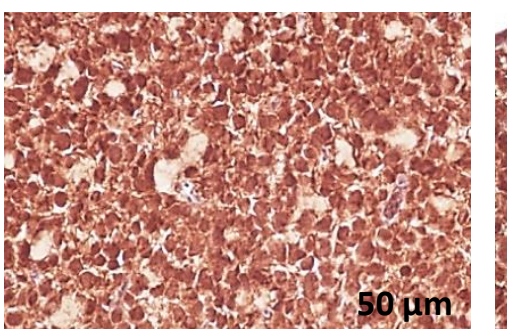

20. $2.250 \mathrm{~mm}$ 2

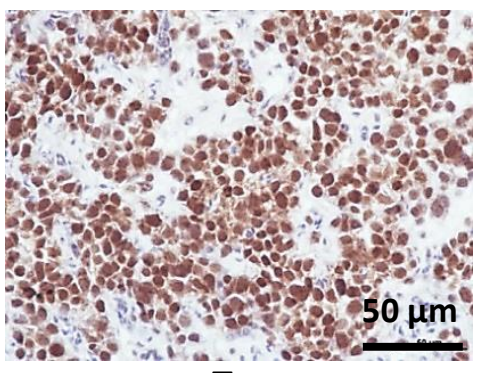

7
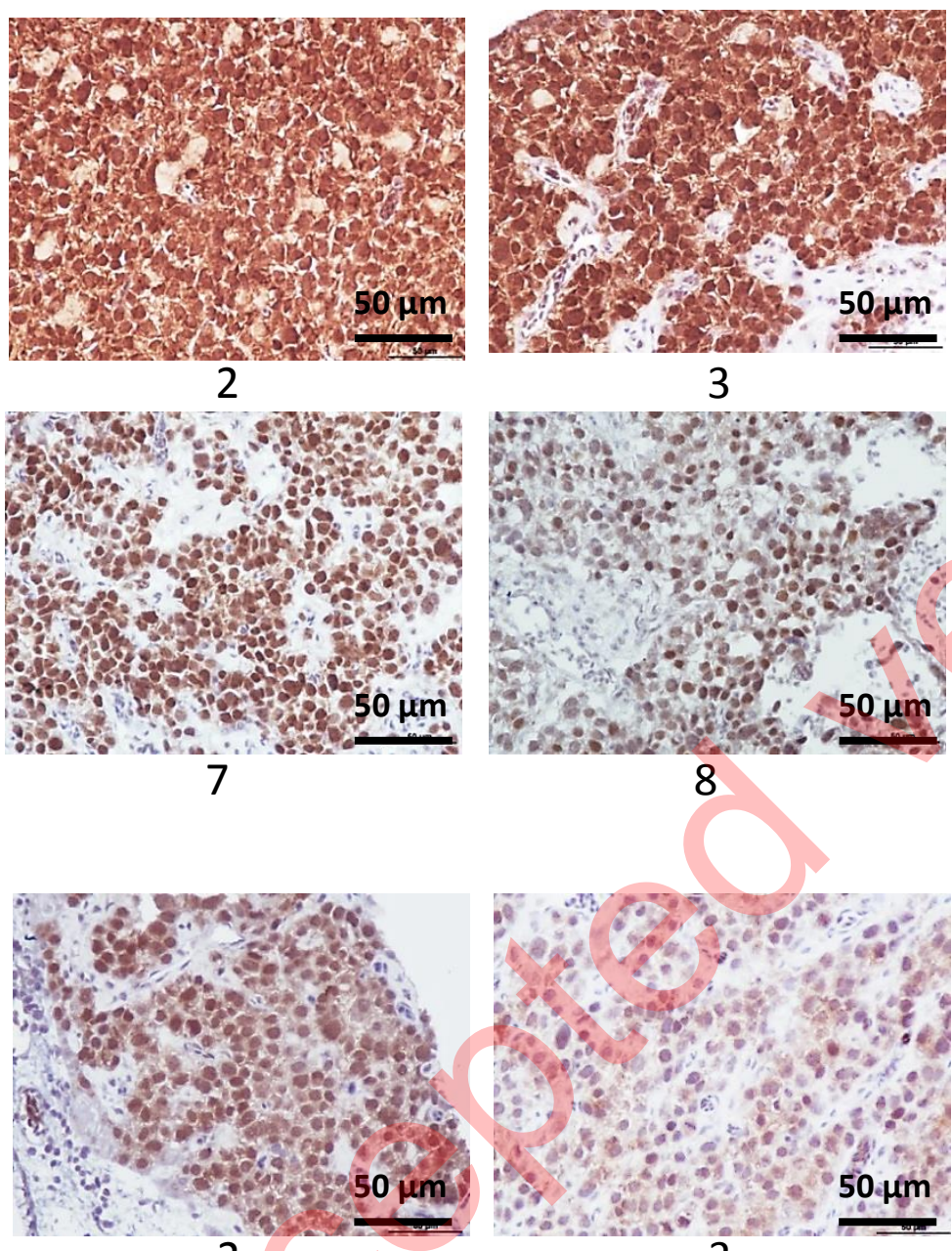
3

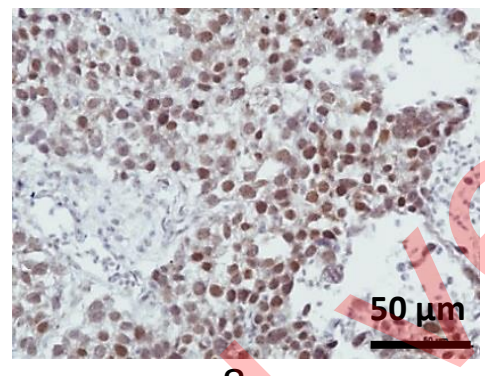

8
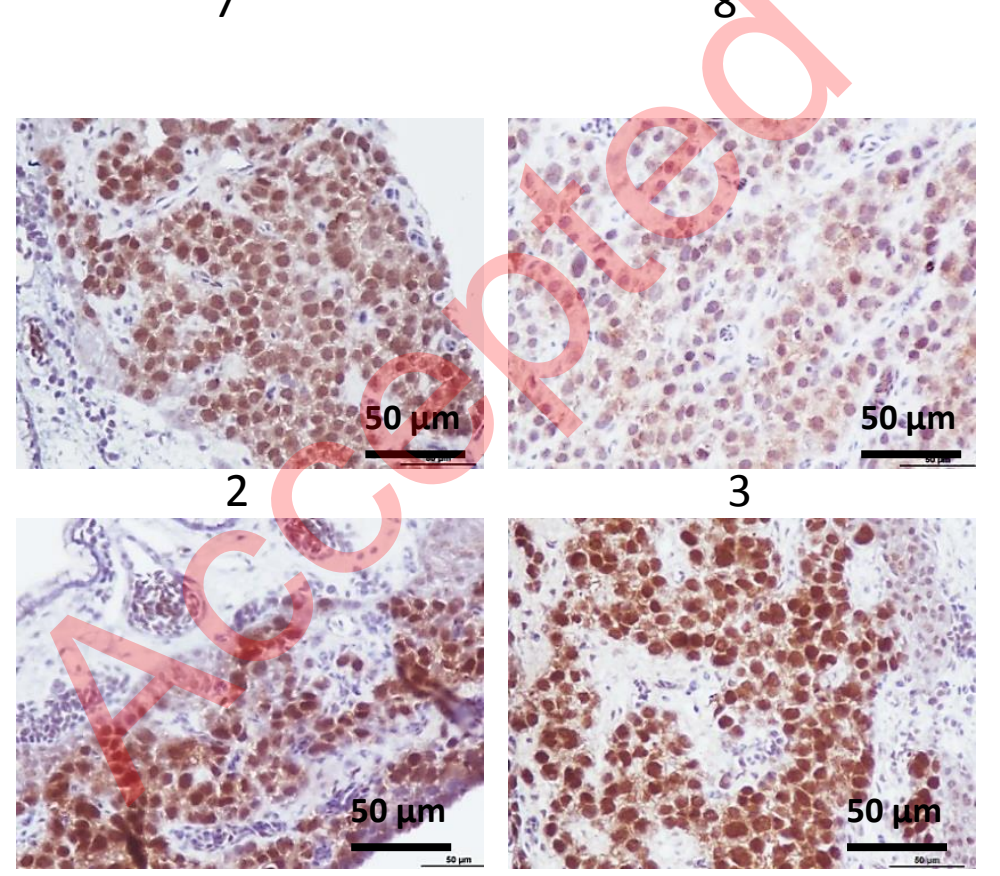

7 3

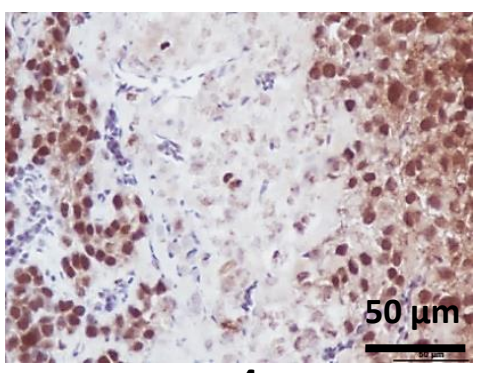
4

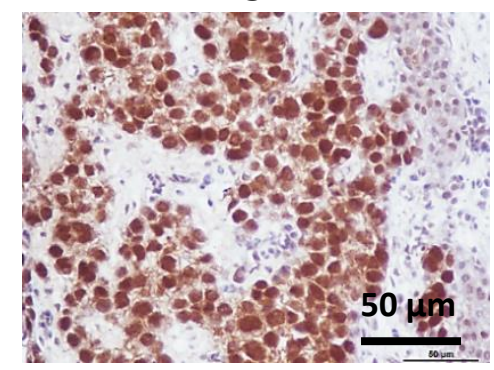

8

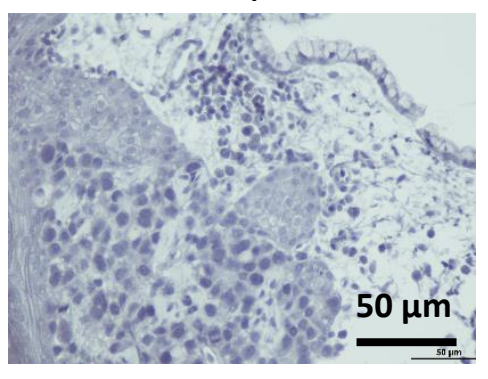

Blank

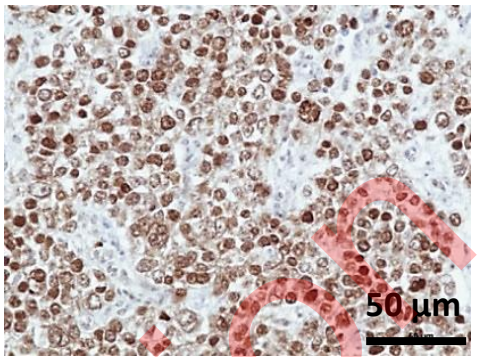

4

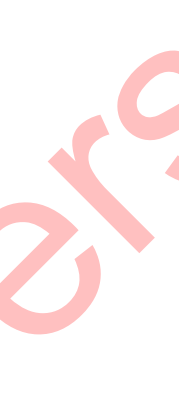

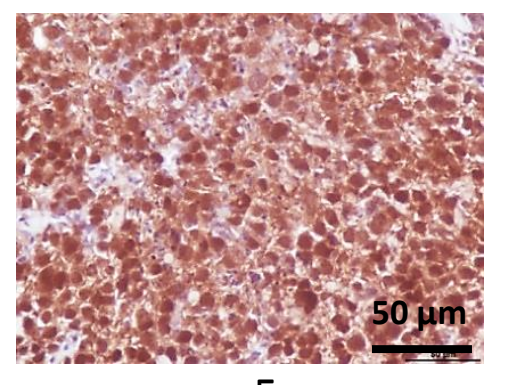

5

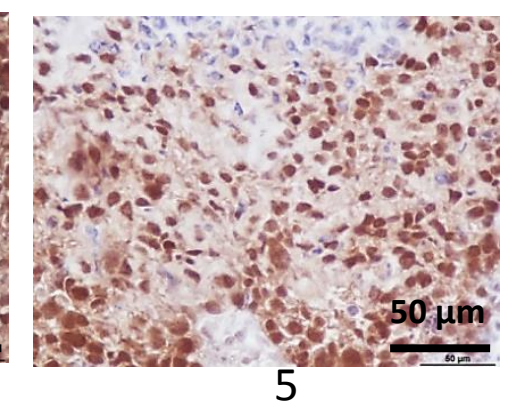

Ghousein A et al, Supplementary Figure S12 
Predicted miR-4510 sites

RAF1 5'UTR (site 1) 291

5' ...UGUGACCGCCUCCCGCUCCCUCAC... 3' 315

miR-4510

3' UUGGUAUGUAGGAUGAGGGAGU 5'

RAF1 5'UTR (site 2)

365

miR-4510

5' ...GUUGGGGCGGCCUGGCUCCCUCAG... 3' 389

| | | | ||

3' UUgGuauguagGaUGAGGGAGU 5'

Ghousein A et al, Supplementary Figure S13 